\title{
How rare are counter Evershed flows?
}

\author{
J. S. Castellanos Durán ${ }^{1,2} \oplus$, A. $\operatorname{Lagg}^{1,4} \oplus$, and S. K. Solanki ${ }^{1,3} \odot$ \\ 1 Max Planck Institute for Solar System Research, Justus-von-Liebig-Weg 3, 37077 Göttingen, Germany \\ e-mail: castellanos@mps.mpg.de \\ 2 Georg-August-Universität Göttingen, Friedrich-Hund-Platz 1, 37077 Göttingen, Germany \\ 3 School of Space Research, Kyung Hee University, Yongin 446-101, Gyeonggi, Republic of Korea \\ ${ }^{4}$ Department of Computer Science, Aalto University, PO Box 15400, 00076 Aalto, Finland
}

Received 23 April 2021 / Accepted 9 June 2021

\section{ABSTRACT}

\begin{abstract}
One of the main characteristics of sunspot penumbrae is the radially outward-directed Evershed flow. Only recently have penumbral regions been reported with similar characteristics to normal penumbral filaments but with an opposite direction of the flow. Such flows directed toward the umbra are known as counter Evershed flows (CEFs). We aim to determine the occurrence frequency of CEFs in active regions (ARs) and to characterize their lifetime and the prevailing conditions in the ARs. We analyzed the continuum images, Dopplergrams, and magnetograms recorded by SDO/HMI of 97 ARs that appeared from 2011 to 2017. We followed the ARs for $9.6 \pm 1.4$ days on average. We found $384 \mathrm{CEFs}$ in total, with a median value of six CEFs per AR. Counter Evershed flows are a rather common feature, occurring in $83.5 \%$ of all ARs regardless of the magnetic complexity of the AR. However, CEFs were only observed, on average, during $5.9 \%$ of the mean total duration of all the observations analyzed here. The lifetime of CEFs follows a log-normal distribution with a median value of $10.6_{-6.0}^{+12.4} \mathrm{~h}$. In addition, we report two populations of CEFs, those that are associated with light bridges and those that are not. We explain that the rarity of reports of CEFs in the literature is a result of highly incomplete coverage of ARs with spectropolarimetric data. By using the continuous observations now routinely available from space, we are able to overcome this limitation.
\end{abstract}

Key words. sunspots - Sun: photosphere

\section{Introduction}

Sunspots are a manifestation of solar magnetism. The two main constituents of sunspots are the dark umbra, which harbors a strong and relatively vertical magnetic field $(B)$, and the penumbra, which is highly filamentary in continuous radiation and has a more horizontal $B$.

A radially outward-directed flow along the penumbra produces Doppler-shifted photospheric spectral lines ${ }^{1}$ when the sunspot is observed away from disk center. This Evershed flow (see Solanki 2003 for a review) was detected more than a century ago (Evershed 1909). In the past decade, there have been a few reports of peculiar flows directed toward the umbra (Kleint \& Sainz Dalda 2013; Louis et al. 2014; Siu-Tapia et al. 2017; Guglielmino et al. 2017, 2019; Louis et al. 2020). These socalled counter Evershed flows (CEFs) have also been observed in one magnetohydrodynamic (MHD) simulation (Siu-Tapia et al. 2018). Counter Evershed flows studied in the literature have been found in complex active regions (ARs). Recently, Louis et al. (2020) reported the appearance of a light bridge associated with a CEF. Common to these works, each of which analyzed only one sunspot, is that CEFs were described as "anomalous," "unusual," or "atypical" flows.

Photospheric CEFs should not be confused with the more commonly reported chromospheric inverse Evershed flows (St. John 1911a,b; Maltby 1975; Choudhary \& Beck 2018; Beck \&

\footnotetext{
1 In this paper the radial direction is taken to be parallel to the solar surface and ascribed from the umbra-penumbra boundary, across the penumbra, toward the quiet-Sun.
}

Choudhary 2019). Inverse Evershed flows transport material in the chromospheric penumbrae toward the umbra and are thought to be driven by pressure gradients (siphon flows; e.g., Thomas 1988).

The few existing reports of CEFs might suggest that this type of photospheric flow is a rare phenomenon in sunspots. However, until now, no study had systematically looked at a large set of ARs to quantify how often CEFs occur in ARs and to determine whether their occurrence depends on the magnetic complexity of the host AR. In this Letter we fill this gap and present the first analysis of CEFs in a large sample of ARs using six years of space-borne data.

\section{Observational data and analysis}

We analyzed 97 ARs observed by the Solar Dynamic Observatory (SDO; Pesnell et al. 2012) taken by the Helioseismic and Magnetic Imager (HMI; Scherrer et al. 2012; Schou et al. 2012). We analyzed the continuum intensity $\left(I_{\mathrm{c}}\right)$, Dopplergrams ( $\left.v_{\mathrm{LOS}}\right)$, and magnetograms $\left(B_{\mathrm{LOS}}\right)$ with a spatial resolution of $1^{\prime \prime}$. Data were taken with a cadence of $12 \mathrm{~min}$ and covered the development of each AR over a time ranging from 153 to $278 \mathrm{~h}$. Table C.1 summarizes the observations. Data were processed using standard SSWIDL routines, and temporal series were created by co-aligning the images using cross-correlations between the subsequent frames.

The 97 ARs were observed between 2011 July 30 and 2017 August 24. Active regions were tracked continuously while they crossed the solar disk, for $9.6 \pm 1.4$ days on average. The only 


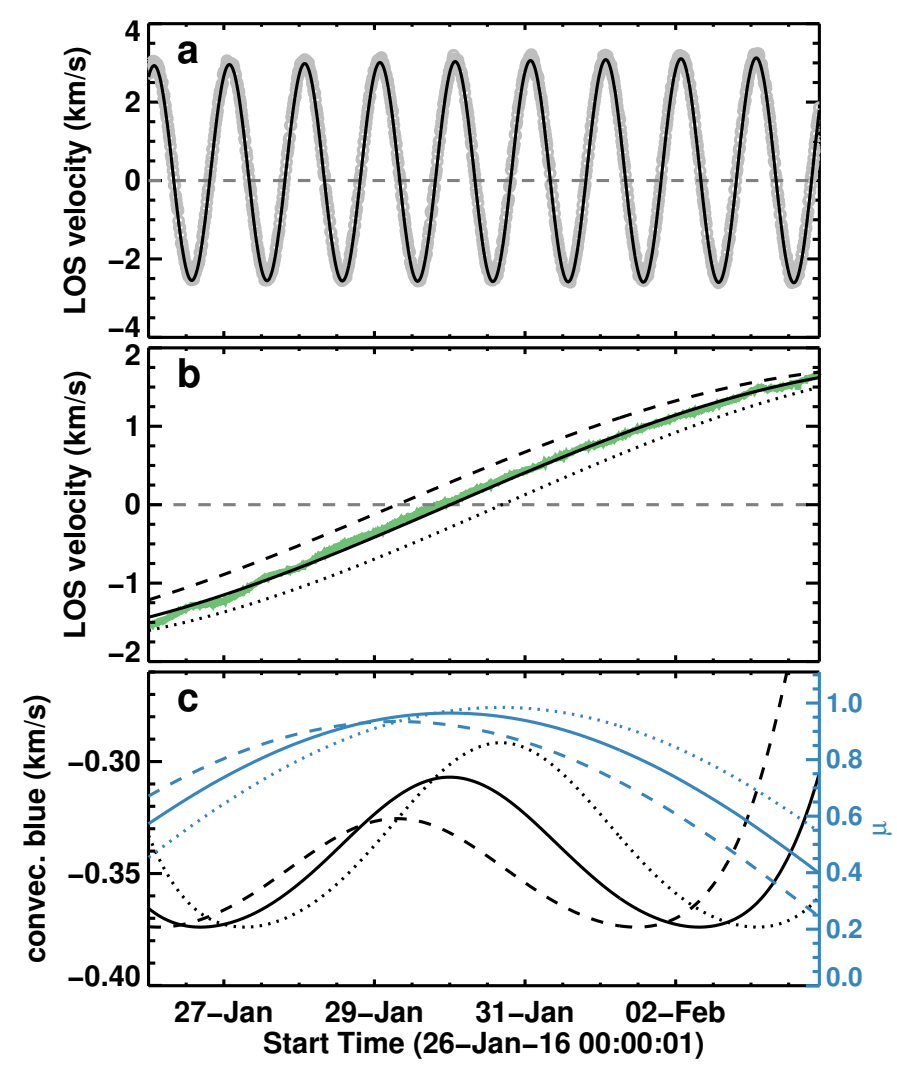

Fig. 1. Examples of the calibration of the Dopplergrams measured by SDO/HMI for AR 12489. (a): average LOS velocities estimated in the quiet-Sun at disk center as a proxy for the observatory velocity, $v_{\mathrm{LOS}}^{\mathrm{SDO}}$ (dotted gray line). The black line is the $v_{\mathrm{LOS}}^{\mathrm{SDO}}$ obtained by Eq. (1). $(b)$ : solar differential rotation obtained from Eqs. (2)-(4), shown with black lines, for the lower-left (dotted line), middle (solid line), and top-right (dashed line) pixels inside a FoV with a size of $310^{\prime \prime} \times 185^{\prime \prime}$. The green line is the average LOS velocity of the adjacent quiet-Sun. (c): convective blueshift estimated using Eq. (5) at the same three pixels inside the FoV, shown with black lines, with the $\mu$ values given by the blue lines.

criteria applied when selecting an AR was that it had sunspots and could be followed for at least six consecutive days, regardless of whether individual sunspots within the AR emerged or decayed during this period. These criteria exclude many small ARs with only small, short-lived sunspots as well as many ARs that formed when already fully on the disc or ARs that were already decaying when they appeared at the east limb. They might also exclude some ARs that appeared during the SDO's eclipsing seasons. We analyzed $\sim 1.1 \times 10^{5}$ individual time steps (for each acquired $I_{\mathrm{c}}, v_{\mathrm{LOS}}$, and $B_{\mathrm{LOS}}$ ).

Our sample covers all classification types of ARs, from the simplest to the most complex. The magnetic complexity of solar ARs is regularly described by Hale's classification (Hale et al. 1919). In this classification, an AR is assigned to the category $\alpha$ if it contains one or multiple sunspots of unique polarity, $\beta$ refers to ARs that harbor bipolar sunspots or groups, and $\gamma$ describes complex ARs with sunspots and groups of intermixed polarities. An amendment to Hale's classification was made in the 1960s with the addition of an extra category, $\delta$, to describe those ARs that harbor umbrae of mixed polarities enclosed by a common penumbra within $<2^{\circ}$ (Künzel 1960, 1965). The categories can be appended to describe the complexity of an AR, increasing from $\alpha$ to $\beta \gamma \delta$. In our sample, we take into account the change in the magnetic classification for each AR over their lifetimes.
Considering that bipolar- $\beta$ ARs are the most common type observed on the Sun ( 65\%; Jaeggli \& Norton 2016), our sample is dominated by this AR type.

The determination of the zero level of the line-of-sight (LOS) velocity measurements requires taking the following effects into account: (1) the LOS velocity of the observatory with respect to the Sun $\left(v_{\mathrm{LOS}}^{\mathrm{SDO}}\right),(2)$ the large-scale flows (LSFs) on the solar surface, including solar differential rotation and meridional circulation, (3) the center-to-limb variation in the convective blueshift, and (4) the gravitational redshift. For SDO, the value for $v_{\mathrm{LOS}}^{\mathrm{SDO}}$ was estimated by combining the keywords OBS_VR, OBS_VW, and OBS_VN in the header of the HMI data files. Following Schuck et al. (2016), the $v_{\text {LOS }}^{\text {SDO }}$ in the helioprojective coordinate system $\left(\theta_{\rho}, \psi\right)$ is given by

$$
\begin{aligned}
v_{\mathrm{LOS}}^{\mathrm{SDO}}= & \text { OBS_VW } \sin \theta_{\rho} \sin \psi \\
& - \text { OBS_VN } \sin \theta_{\rho} \cos \psi+\text { OBS_VR } \cos \theta_{\rho},
\end{aligned}
$$

where these keywords provide information about the speed of the observatory in the radial direction from the Sun (OBS_VR), westward in the direction of Earth's orbit (OBS_VW), and northward in the direction of solar north (OBS_VN). Thompson (2006) provides the conversion between coordinate systems.

An alternative method to account for effect (1) is based on the HMI Doppler- and magnetograms to estimate the average quiet-Sun velocity calculated from a region $\pm 15^{\prime \prime}$ around disk center. Strong magnetic concentrations are avoided by masking regions with $\left|B_{\mathrm{LOS}}\right|>500 \mathrm{G}$. The good agreement between the average quiet-Sun velocities and the velocity information taken from the header of the data files can be gleaned from Fig. 1a, which shows diurnal variations in the spacecraft velocity of up to $\pm 3 \mathrm{~km} \mathrm{~s}^{-1}$. The peak-to-peak amplitude and mean of the $v_{\mathrm{LOS}}^{\mathrm{SDO}}$ change over the course of the year.

The correction for the LSFs (effect 2) has two components: differential solar rotation $\left(v_{\text {rot }_{\odot}}\right)$ and the surface meridional flow $\left(v_{\text {mer }_{\odot}}\right)$. We calculated their contribution at every pixel in our maps based on its Stonyhurst heliographic coordinates latitude and longitude $(\Theta, \Phi)$. The LOS component of these LSFs, for $\theta_{\rho} \approx 0^{2}$, is given by

$$
\begin{aligned}
\left.v_{\mathrm{LSF}_{\odot}}(\Phi, \Theta)\right|_{\mathrm{LOS}}= & {\left[v_{\operatorname{med}_{\odot}}(\Theta)\right]\left[\sin B_{0} \cos \Theta-\cos B_{0} \cos \Phi \sin \Theta\right] } \\
& -\left[v_{\text {rot }_{\odot}}(\Theta)-v_{\text {Carrington }}+v_{\text {synodic }}\right] \cos B_{0} \sin \Phi,
\end{aligned}
$$

where $v_{\text {Carrington }}=14.184 \mathrm{deg} \mathrm{day}^{-1}$ is the Carrington rotation rate, corresponding to a velocity of $1994.21 \mathrm{~m} \mathrm{~s}^{-1}$ at the equator. The $v_{\text {synodic }}=0.986 \mathrm{deg} \mathrm{day}^{-1}$ is the mean orbital angular velocity of the observer to account for the synodic rotation between the Sun and Earth. At the equator, $v_{\text {synodic }}=138.63 \mathrm{~m} \mathrm{~s}^{-1}$. The differential solar rotation is given by

$v_{\text {rot }_{\odot}}(\Theta)=\left(a+b \sin ^{2} \Theta+c \sin ^{4} \Theta\right) \cos \Theta\left[\mathrm{m} \mathrm{s}^{-1}\right]$,

and the surface meridional flow is given by

$v_{\text {mer } \odot}(\Theta)=\left(\mathrm{d} \sin \Theta+e \sin ^{3} \Theta\right) \cos \Theta\left[\mathrm{m} \mathrm{s}^{-1}\right]$.

The coefficients in Eqs. (3) and (4) are $a=35.6 \mathrm{~m} \mathrm{~s}^{-1}, b=$ $-208.6 \mathrm{~m} \mathrm{~s}^{-1}, c=-420.6 \mathrm{~m} \mathrm{~s}^{-1}, d=29.7 \mathrm{~m} \mathrm{~s}^{-1}$, and $e=$ $-17.7 \mathrm{~m} \mathrm{~s}^{-1}$, as obtained by Hathaway \& Rightmire (2011). The

2 See Eqs. (39a)-(39c) in Schuck et al. (2016) for observations taken closer to the Sun (e.g., by Solar Orbiter), where the maximum value of $\theta_{\rho}$, which is obtained at the limb $\sin \theta_{\rho}=R_{\odot} / D_{\odot}$, is not negligible. 
contribution of the surface meridional flow is tiny and therefore negligible. Figure 1b shows examples of the correction for differential rotation for the bottom-left, center, and top-right pixels within the field-of-view (FoV) for AR 12489. For comparison, the green line shows the average quiet-Sun velocity within the FoV. It should be noticed that this average is influenced by the location of the sunspots within the AR inside the observed FoV, which may change as they evolve and change shape over multiple days.

To compensate for the center-to-limb variation in the convective blueshift, we reconstructed the profile from LöhnerBöttcher \& Schlichenmaier (2013, their Fig. 2c) and shifted the profile to match it with the convective blueshift value at disk center as measured by the Laser Absolute Reference Spectrograph (LARS; Doerr 2015; Löhner-Böttcher et al. 2017). LARS performed absolute wavelength-calibrated observations with a resolving power of $\sim 700000$ at $6173 \AA$, determining the convective blueshift for the FeI6173.3 $\AA$ line at disk center to be $-320 \mathrm{~m} \mathrm{~s}^{-1}$ (Stief et al. 2019). At HMI's resolving power of $\sim 81000$, this value reduces to $-275 \mathrm{~m} \mathrm{~s}^{-1}$ (Löhner-Böttcher et al. 2019). Similar to Stief et al. (2019), we fit the center-tolimb variation with a fifth-degree polynomial, given by

$$
\begin{aligned}
v_{\mathrm{CLV}}(\mu)= & 134-1179 \mu-2029 \mu^{2} \\
& +9112 \mu^{3}-10409 \mu^{4}+4096 \mu^{5}\left[\mathrm{~m} \mathrm{~s}^{-1}\right],
\end{aligned}
$$

where $\mu=\cos \theta$ and $\theta$ is the heliocentric angle. To correct for this effect, we obtained the $\mu$ values for all pixels inside the FoV and subtracted the result of Eq. (5) from the $v_{\text {LOS }}$. An example of this correction, taken at the same three pixels inside the FoV, is shown in Fig. 1c.

The gravitational redshift is a relativistic effect and a direct application of the equivalence principle for the light traveling from the Sun to the Earth. The gravitational Doppler shift is given by

$\Delta \lambda_{\mathrm{G}}=\lambda G m_{\odot} / R_{\odot} c^{2} \therefore v_{\mathrm{G}}=636.03\left[\mathrm{~m} \mathrm{~s}^{-1}\right]$,

where all the constants have their nominal meanings.

Continuum images were corrected for limb darkening following the procedure explained in Castellanos Durán \& Kleint (2020). To select the penumbra, we used the continuum images and set the commonly used limits $0.5 I_{\mathrm{qs}} \leq I_{\mathrm{penumbra}} \leq 0.97 I_{\mathrm{qs}}$, where $I_{\mathrm{qs}}$ is the mean quiet-Sun intensity.

Due to the Evershed flow, the limb-side penumbra is observed redshifted, while the center-side penumbra is blueshifted. This pattern is reversed if a sector of the penumbra is carrying a CEF. A CEF appears blueshifted if it is located on the limb-ward side and redshifted on the center side. When searching for candidate CEFs, we added the constraint that these events should appear in penumbrae and that the penumbral region should have an adjacent umbra. The second condition avoids orphan penumbrae that tend to show strong flows but for which it is nontrivial to establish whether the flow direction corresponds to the normal Evershed flow or not.

Candidate CEFs were found using the SOBEL edgeenhancement and CANNY edge-detection algorithms, both implemented in the Interactive Data Language IDL8. 3 (Exelis Visual Information Solutions, Boulder, Colorado), to detect breaks in the Dopplergrams within the penumbrae. In addition, we calculated the spatial gradient of $v_{\mathrm{LOS}}$, where the position of the maximum gradient often outlines the CEF region very well. However, this method sometimes failed, especially when the CEF was orientated closely parallel to the direction of the closest limb. It is

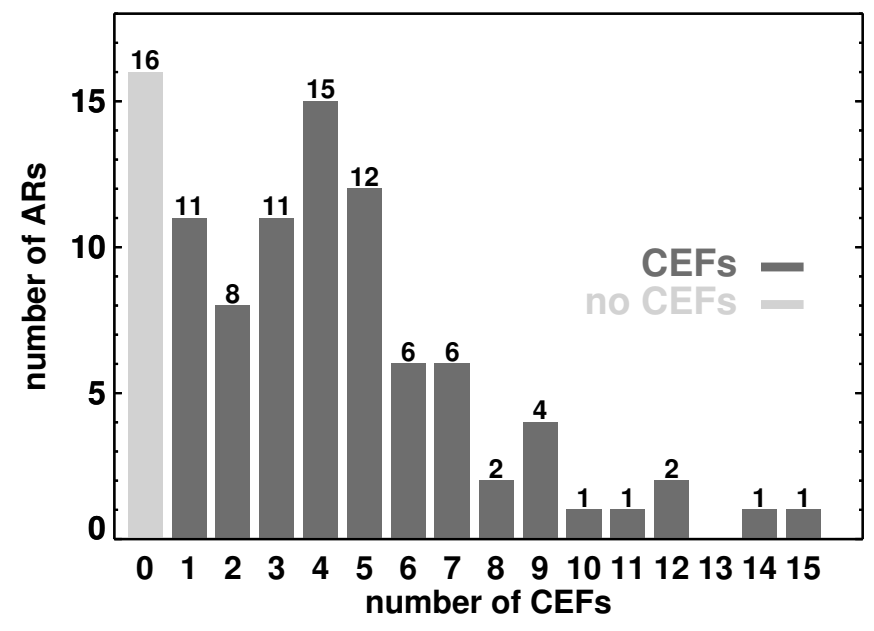

Fig. 2. Number of CEFs per AR. The median value of CEFs per AR is six. An overview of the 16 ARs without any CEF is presented in Appendix A.

difficult to detect CEFs that are aligned within $10-15^{\circ}$ of the direction of the nearest solar limb (these flows are perpendicular to the LOS and hence produce almost no Doppler shift). We, therefore, must have missed between $5 \%$ and $10 \%$ of all the CEFs in the studied ARs if we assume that CEFs are isotropically distributed. We additionally checked all sunspots within the ARs, by visual inspection of videos that showed their temporal evolution, for CEFs that were not identified by the method described above.

We used the Solar Region Summary (SRS) provided by the Space Weather Prediction Center $\left(\mathrm{SWPC}^{3}\right)$ to determine parameters such as the NOAA AR number, the heliographic latitude and longitude, the area of the AR, and the magnetic classification of the AR. We completed the records for a few ARs that were excluded from the SRS reports because they emerged on the same day, but after the SRS reports were issued at 00:30 UT.

\section{Results}

We found CEFs in 81 out of the 97 ARs studied between 2011 July 30 and 2017 August 24. Figure 2 shows the distribution of the numbers of CEFs per AR. The median number of CEFs detected per AR is six. While eleven ARs presented just one $\mathrm{CEF}$, the peak is found at four CEFs, with fifteen ARs harboring that number. There are six ARs with ten or more CEFs, with AR 11520 harboring fifteen CEFs (see Fig. B.69), the largest number for any AR in our sample. Some CEFs also appear in nests, that is to say, multiple CEFs are observed to form in the same part of the penumbra of a given sunspot. In a few cases, multiple CEFs were observed at the same time at different locations of the same sunspot. We observed that the occurrence of CEFs depends neither on the size nor on the number of sunspots belonging to an AR. Table C.1 summarizes the parameters of the observations and the general properties of the hosting ARs, such as the NOAA AR, the date and time, the total duration of the observation, the size of the FoV, the median latitude of the AR, the start and end longitude of the considered observations of the $\mathrm{AR}$, and the magnetic classification of the AR on all the days it was observed.

Four examples of CEFs are shown in Fig. 3 (from left to right): the continuum image of the whole AR and a zoomed

3 https://wWW.swpc.noaa.gov/ 


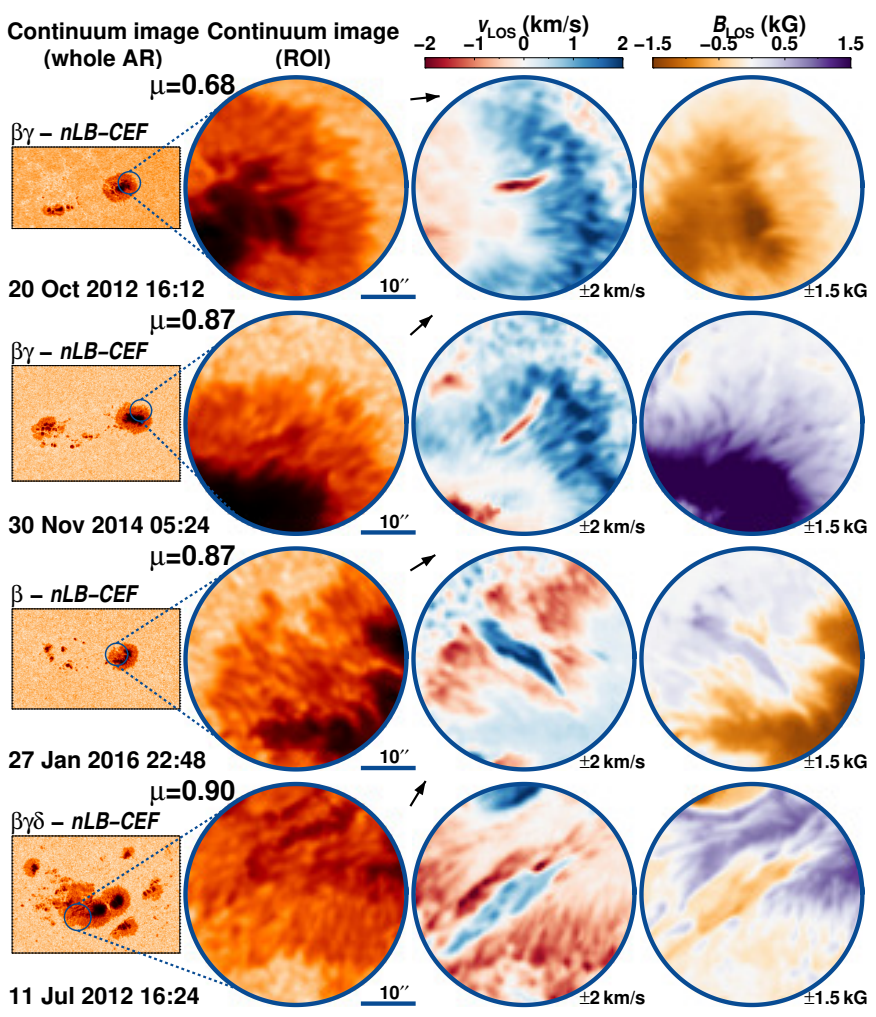

Fig. 3. Examples of CEFs not associated with light bridges (nLB-CEFs) found in four ARs. In every row, the left-most map is the continuum image of the whole AR, and the three next maps present a close-up of the region surrounding the CEF. From left to right they are: the continuum image, the Dopplergram clipped at $\pm 2 \mathrm{~km} \mathrm{~s}^{-1}$, and the magnetogram clipped at $\pm 1.5 \mathrm{kG}$. Arrows point toward disk center. The magnetic classification of the AR and the time of the observation are shown at the top-left and bottom-left of each row.

in view of three regions of interest showing three quantities, $I_{\mathrm{c}}, v_{\mathrm{LOS}}$, and $B_{\mathrm{LOS}}$. Counter Evershed flows generally appear as elongated structures that, depending on their location, are either redshifted (Fig. 3; two top rows) or blueshifted (Fig. 3; two bottom rows). All the CEFs found in this study are presented in Figs. B.1-B.81. Each figure groups all CEFs harbored within one AR. The layout of these figures is the same as in Fig. 3.

We find that CEFs can be categorized into two different groups. The first type are CEFs associated with light bridges: Such CEFs originate in a light bridge or in a penumbral intrusion (i.e., a partial light bridge) and extend into the penumbra with an opposite flow direction compared to that of the adjacent penumbra. We label these CEFs as "LB-CEFs." Four examples of such LB-CEFs are plotted in Fig. 4. The second type are CEFs that are completely embedded in the penumbra, labeled as "nLB-CEFs" (non-light bridge CEFs; Fig. 3). Both types of CEFs are almost equally common in our sample: $198(51.6 \%)$ nLB-CEFs versus 186 (48.4\%) LB-CEFs.

Counter Evershed flows appeared in all magnetic types of ARs: ranging from simple $\alpha$ ARs to the more complex $\delta$ ARs. Similarly, the few ARs not harboring any CEF also show many complexity categories. The examples of CEFs presented in Figs. 3 and 4 indicate the magnetic classification just above the leftmost image of each row, illustrating the large variety of ARs where CEFs appeared. There is no clear dependence of the occurrence of CEFs on the complexity of the AR. Both types of CEFs appear in all magnetic classes.

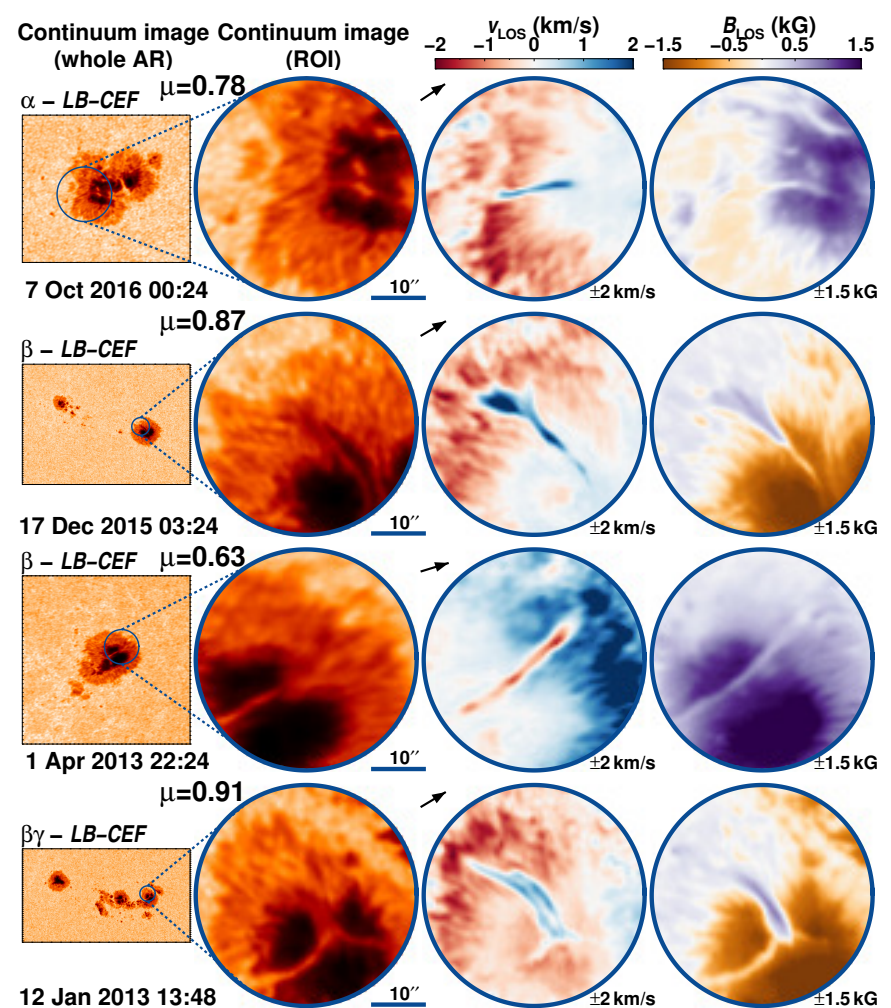

Fig. 4. Examples of four CEFs associated with light bridges (LB-CEFs); same layout as Fig. 3.

The 81 ARs that hosted CEFs were observed for a total of $\sim 1000$ days. The percentage of days on which CEFs are observed does seem to depend on the magnetic complexity of an AR. For the simplest regions (i.e., $\alpha$ regions), CEFs are visible on only $8 \%$ of all days. This increases to roughly one-third of all days for $\beta$ and $\beta \gamma$ regions and finally becomes slightly more than $50 \%$ for the $\beta \gamma \delta$ regions.

We did not observe CEFs in 16 ARs (16.5\%), all but one of which contained only a single sunspot. The magnetic classification of these ARs is dominated by the simple class, $\alpha$ ( $51.8 \%$ of all days these regions were observed). In $39.3 \%$ of those observed days, the ARs without CEFs appear in $\beta$ configuration, $7.1 \%$ in $\beta \gamma$, and $1.8 \%$ in $\beta \delta$. Figure A.1 shows the continuum images of all the ARs without CEFs. The sunspots within these ARs share a similar roundish morphology and are all rather small, with a diameter smaller than $40^{\prime \prime}$. The details of these ARs, as well as their evolution through different magnetic classes, are summarized in the top part of Table C. 1 .

The lifetimes of the CEFs found in the literature range from $1 \mathrm{~h}$ to approximately $12 \mathrm{~h}$ (e.g., Louis et al. 2014, 2020; SiuTapia et al. 2018). Figure 5 presents the histogram of the lifetimes of the CEFs from our study. The median and average lifetime of CEFs are $10.6_{-6.0}^{+12.4} \mathrm{~h}$ and $13.5 \mathrm{~h}$, respectively. The total lifetime distribution is reasonably well reproduced by a lognormal function given by

$f_{\log -\text { normal }}(t)=\frac{\eta}{\xi \sqrt{2 \pi} t} \exp \left[-\frac{[\ln (t / \zeta)]^{2}}{2 \xi^{2}}\right]$,

where $\eta=780.6 \pm 0.6, \zeta=11.6 \pm 0.4$, and $\xi=0.8 \pm 0.03$. The peak in the lifetime distribution is found at around $\sim 7 \mathrm{~h}$, and $85.1 \%$ of CEFs have a lifetime shorter than 1 day. The previously reported lifetimes of CEFs fall close to the median value, including the MHD-simulated CEF that vanishes after $\sim 10 \mathrm{~h}$ 


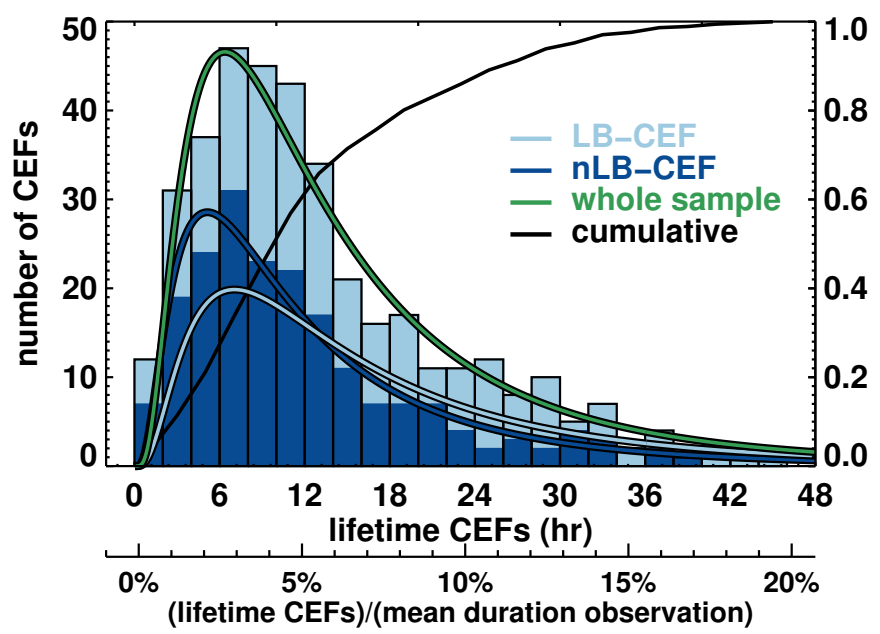

Fig. 5. Lifetime of CEFs. The maximum is $\sim 7 \mathrm{~h}$, and the median is $10.6_{-6.0}^{+12.4} \mathrm{~h}$. Colors mark nLB-CEFs (dark blue) and LB-CEFs (light blue). Thick lines show the best fit of a log-normal function (Eq. (7)) to the distributions of nLB-CEFs (dark blue), LB-CEFs (light blue), and the whole sample (green line). The cumulative curve is shown in black.

(Siu-Tapia et al. 2018). On average, we found a CEF in $5.9 \%$ of the mean total duration of the observations of an AR (see the bottom $x$-axis in Fig. 5).

In Fig. 5 we differentiate between nLB-CEFs (dark blue) and LB-CEFs (light blue). The mean lifetime of LB-CEFs is $3.5 \mathrm{~h}$ longer $(15.4 \pm 10.1 \mathrm{~h}$ compared to $11.9 \pm 9.3 \mathrm{~h})$. In addition, the lifetime of CEFs does not depend on the magnetic classification of the AR (not shown).

We found a slight positive trend between the lifetime of CEFs and the total area of the AR but with a large scatter, so the trend is not statistically significant at the $1 \sigma$ confidence level. In addition, ARs are usually formed by a group of many sunspots (e.g., Mandal et al. 2021), while CEFs often appear in just one of the sunspots within the AR. Therefore, we divided the total area of the AR by the number of visible sunspots belonging to the AR. The scatter remains large. The slight positive trend is enhanced but still not statistically significant.

Most of the CEFs appear as elongated structures, with the $B_{\mathrm{LOS}}$ values being lower than in the surrounding penumbra and sometimes even of opposite magnetic polarity (see for example Fig. 4, cf. Lim et al. 2020). As we used LOS magnetograms for this study, this polarity statement is only reliable for CEFs located at $\mu$ values larger than $\approx 0.8$. From the $181 \mathrm{CEFs}$ fulfilling this criterion, $\sim 42 \%$ are of opposite polarity compared to the surrounding penumbra (38/91 nLB-CEF versus 38/90 LB-CEF).

\section{Summary and conclusions}

We have presented the first statistical survey of CEFs in a sample of 97 ARs. We tracked the ARs for $9.6 \pm 1.4$ days on average using the continuous observations by SDO/HMI. We report the appearance of 384 CEFs in $83.5 \%$ of the ARs. Counter Evershed flows were observed in all magnetic types of the ARs cataloged, from $\alpha$ and $\beta$ to $\beta \gamma \delta$. The number of CEFs produced by an AR ranges from one to fifteen, with a median value of six CEFs per AR.

We distinguished two populations of CEFs, one associated with light bridges and the other not. The two populations are almost equal in size, and approximately two-fifths of the CEFs within these two populations are of opposite polarity with respect to the surrounding penumbra. Given that not all sunspots have light bridges and that the light bridges take up only a small part of the sunspot, we conclude that light bridges provide favorable conditions for the formation of CEFs. The LB-CEFs have slightly longer lifetimes, but the separation of these two populations based on their lifetimes is not statistically significant.

Almost all the CEFs in our sample are long and narrow. We could not find another example as broad (i.e., covering such a large penumbral segment) as the CEF reported by Siu-Tapia et al. (2017). These results enhance the uniqueness of AR 11930 and its CEF (Siu-Tapia et al. 2019).

The paucity of reports in the literature of ARs associated with CEFs suggests that this might be a rather rare phenomenon. However, in this survey we have shown that almost all ARs of all magnetic types harbor CEFs. The reason for this seeming discrepancy most likely lies in the fact that CEFs are narrow and can easily be missed. Additionally, the average lifetime of CEFs is rather short compared to the total duration of the observation. The ratio between these two quantities highlights the observational difficulty of detecting CEFs (see the bottom $x$-axis in Fig. 5): we needed to continuously track the ARs for, on average, $9.6 \pm 1.4$ days to detect six CEFs in an AR (median value). From our findings, we expect to only see a CEF during an average of $5.9 \%$ of the mean total duration of the observation of an AR. Therefore, to detect CEFs, uninterrupted and stable time series of continuum images and Doppler velocity observations are required. These requirements had not been met until observations became available from space, in particular by HMI.

In this survey we have shown that CEFs are far more common than the few cases reported in the literature would suggest. We find that $\sim 85 \%$ of ARs harbor at least one CEF, and typically six, over the course of their lifetimes. However, only HMI provides the uninterrupted, stable, and relatively high-resolution velocity measurements needed to identify a high fraction of CEFs. Based on the probability found in this study, the Japanese solar mission Hinode (Kosugi et al. 2007) should have observed more CEFs than those found in the current literature. A quick and simple search in the archive of the Spectro-Polarimeter (SP) instrument (Ichimoto et al. 2008) revealed 18 CEFs, indicating that the estimates for the occurrence of CEFs derived in this study are reasonable. The regions harboring CEFs observed by Hinode/SP were classified from $\alpha$ to $\delta$, and the CEFs belonging to the two categories (LB-CEFs and nLB-CEFs) are roughly equal in number, consistent with the results reported in this Letter.

The Polarimetric and Helioseismic Imager (PHI; Solanki et al. 2020) on board Solar Orbiter (Müller et al. 2020) will allow us to track individual ARs for longer periods of time thanks to the partial corotation of the spacecraft with the Sun near its perihelion and the possibility of combining measurements from PHI, Hinode/SP, and HMI. Such measurements will allow us to accurately measure the flow velocity and the magnetic and thermodynamic conditions inside CEFs.

Acknowledgements. We thank P.-L. Poulier and H.-P. Doerr for fruitful discussions on the different effects when calibrating HMI's Dopplergrams. J. S. Castellanos Durán would like to show his gratitude to Y. Katsukawa for all the support and invaluable discussions during the visits to the National Astronomical Observatory of Japan (NAOJ). We thank Zhi-Chao Liang and R. Burston for their help with the NetDRMS data management system at MPS. J. S. Castellanos Durán was funded by the Deutscher Akademischer Austauschdienst (DAAD) and the International Max Planck Research School (IMPRS) for Solar System Science at the University of Göttingen. This project has received funding from the European Research Council (ERC) under the European Union's Horizon 2020 research and innovation program (grant agreement No. 695075). The 
data were processed at the German Data Center for SDO (GDC-SDO), funded by the German Aerospace Center (DLR). The HMI data used are courtesy of NASA/SDO and the HMI science team.

\section{References}

Beck, C., \& Choudhary, D. P. 2019, ApJ, 874, 6

Castellanos Durán, J. S., \& Kleint, L. 2020, ApJ, 904, 96

Choudhary, D. P., \& Beck, C. 2018, ApJ, 859, 139

Doerr, H. P. 2015, Ph.D. Thesis, University of Freiburg, Germany

Evershed, J. 1909, MNRAS, 69, 454

Guglielmino, S. L., Romano, P., \& Zuccarello, F. 2017, ApJ, 846, L16

Guglielmino, S. L., Romano, P., Ruiz Cobo, B., Zuccarello, F., \& Murabito, M. 2019, ApJ, 880, 34

Hale, G. E., Ellerman, F., Nicholson, S. B., \& Joy, A. H. 1919, ApJ, 49, 153

Hathaway, D. H., \& Rightmire, L. 2011, ApJ, 729, 80

Ichimoto, K., Lites, B., Elmore, D., et al. 2008, Sol. Phys., 249, 233

Jaeggli, S. A., \& Norton, A. A. 2016, ApJ, 820, L11

Kleint, L., \& Sainz Dalda, A. 2013, ApJ, 770, 74

Kosugi, T., Matsuzaki, K., Sakao, T., et al. 2007, Sol. Phys., 243, 3

Künzel, H. 1960, Astron. Nachr., 285, 271

Künzel, H. 1965, Astron. Nachr., 288, 177

Lim, E.-K., Yang, H., Yurchyshyn, V., et al. 2020, ApJ, 904, 84

Löhner-Böttcher, J., \& Schlichenmaier, R. 2013, A\&A, 551, A105

Löhner-Böttcher, J., Schmidt, W., Doerr, H. P., et al. 2017, A\&A, 607, A12
Löhner-Böttcher, J., Schmidt, W., Schlichenmaier, R., Steinmetz, T., \& Holzwarth, R. 2019, A\&A, 624, A57

Louis, R. E., Beck, C., Mathew, S. K., \& Venkatakrishnan, P. 2014, A\&A, 570, A92

Louis, R. E., Beck, C., \& Choudhary, D. P. 2020, ApJ, 905, 153

Maltby, P. 1975, Sol. Phys., 43, 91

Mandal, S., Krivova, N. A., Cameron, R., \& Solanki, S. K. 2021, A\&A, in press, https://doi.org/10.1051/0004-6361/202140621

Müller, D., St. Cyr, O. C., Zouganelis, I., et al. 2020, A\&A, 642, A1

Pesnell, W. D., Thompson, B. J., \& Chamberlin, P. C. 2012, Sol. Phys., 275, 3

Scherrer, P. H., Schou, J., Bush, R. I., et al. 2012, Sol. Phys., 275, 207

Schou, J., Scherrer, P. H., Bush, R. I., et al. 2012, Sol. Phys., 275, 229

Schuck, P. W., Antiochos, S. K., Leka, K. D., \& Barnes, G. 2016, ApJ, 823, 101

Siu-Tapia, A., Lagg, A., Solanki, S. K., van Noort, M., \& Jurčák, J. 2017, A\&A, 607, A36

Siu-Tapia, A. L., Rempel, M., Lagg, A., \& Solanki, S. K. 2018, ApJ, 852, 66 Siu-Tapia, A., Lagg, A., van Noort, M., Rempel, M., \& Solanki, S. K. 2019, A\&A, 631, A99

Solanki, S. K. 2003, A\&ARv., 11, 153

Solanki, S. K., del Toro Iniesta, J. C., Woch, J., et al. 2020, A\&A, 642, A11

St. John, C. E. 1911a, ApJ, 34, 131

St. John, C. E. 1911b, ApJ, 34, 57

Stief, F., Löhner-Böttcher, J., Schmidt, W., Steinmetz, T., \& Holzwarth, R. 2019, A\&A, 622, A34

Thomas, J. H. 1988, ApJ, 333, 407

Thompson, W. T. 2006, A\&A, 449, 791 


\section{Appendix A: ARs without CEFs}

Counter Evershed flows appear to be a more common phenomenon than previously conceived. We were able to detect CEFs in all but $16.5 \%$ of the ARs in our sample. Continuum images of the 16 ARs without CEFs are presented in Fig. A.1. Most of these ARs are composed of just one sunspot. The top part of Table C.1 summarizes the general characteristics of the ARs without CEFs.

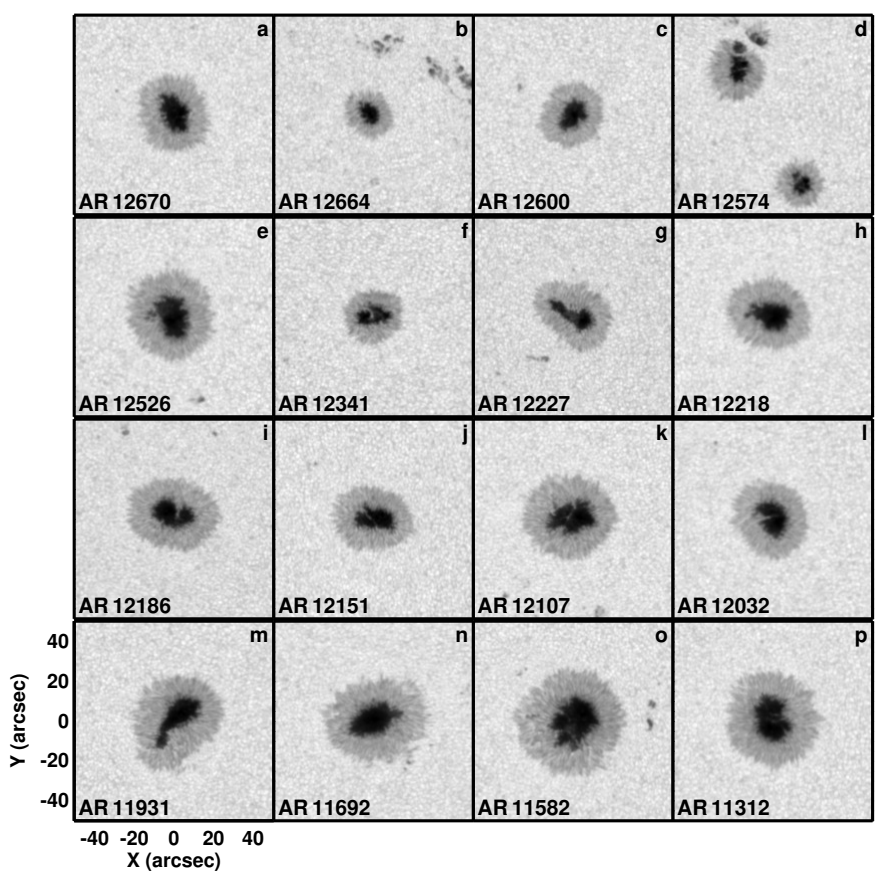

Fig. A.1. Continuum images of the ARs without CEFs. Images were taken when the ARs were close to disk center. 


\section{Appendix B: All CEFs}

All the CEFs found in this study are presented in Figs. B.1-B.81. Each figure groups all the CEFs found per AR. Figures are in reverse chronological order, going from 2017 to 2011. The layout is the same as in Fig. 3. We remark that CEFs are displayed at the time closest to their maximum contrast with respect to their surroundings in the LOS velocity maps. Hence, for a few cases, the association with light bridges and the LB-CEFs are buried.

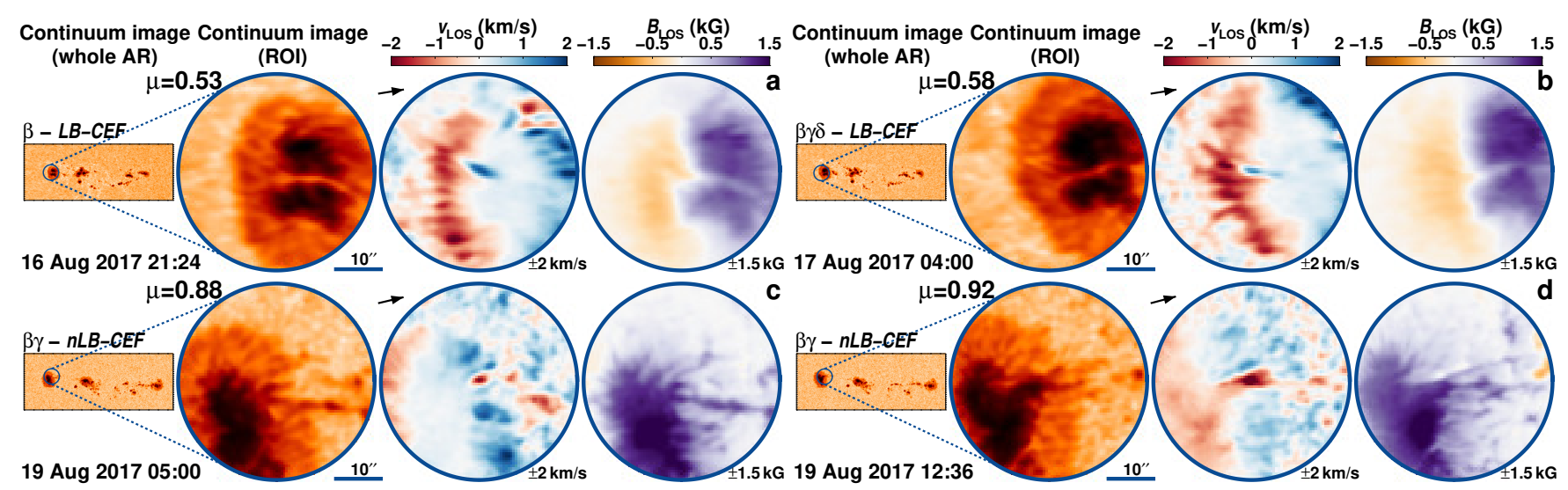

Fig. B.1. AR 12671 followed for 8.6 days from 16-Aug-2017 starting at 06:12 UT.

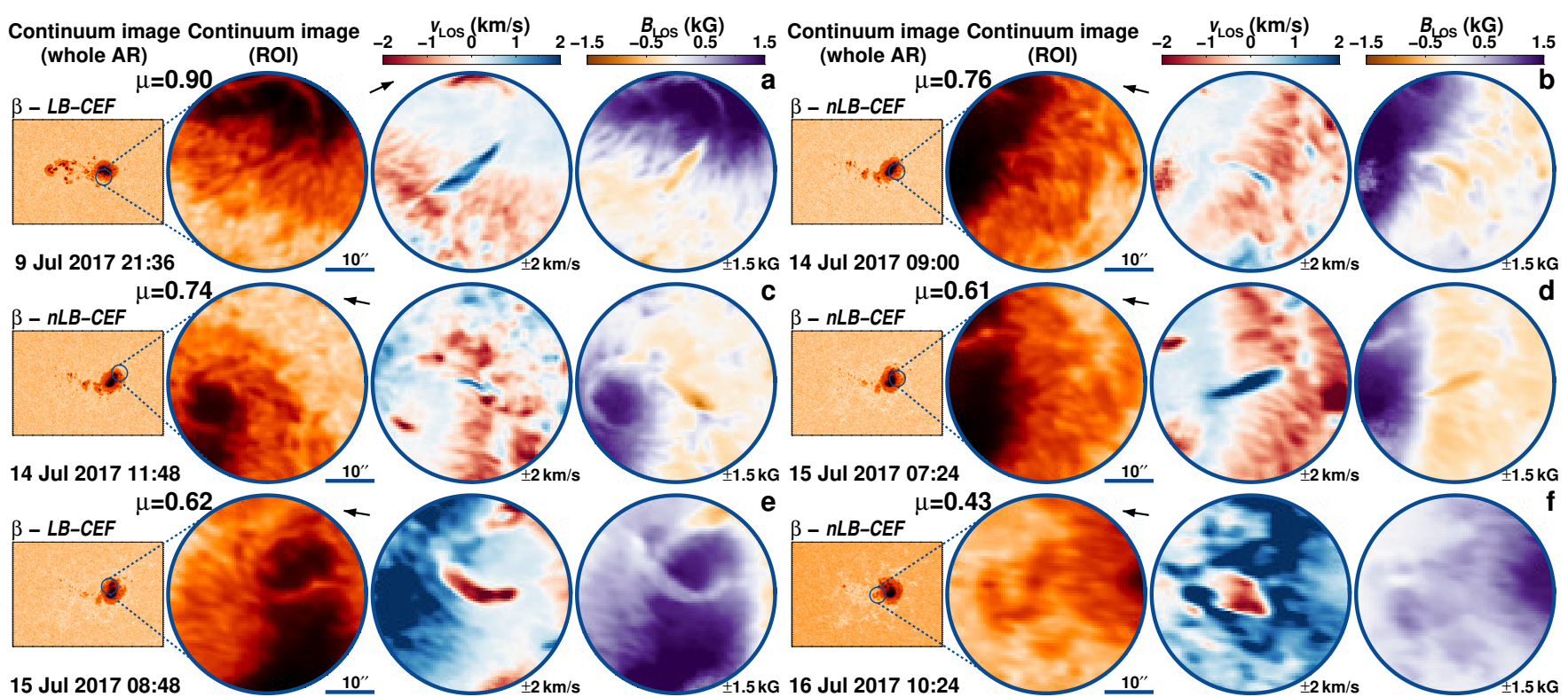

Fig. B.2. AR 12665 followed for 9.4 days from 7-Jul-2017 starting at 12:00 UT. 
J. S. Castellanos Durán et al.: How rare are counter Evershed flows?

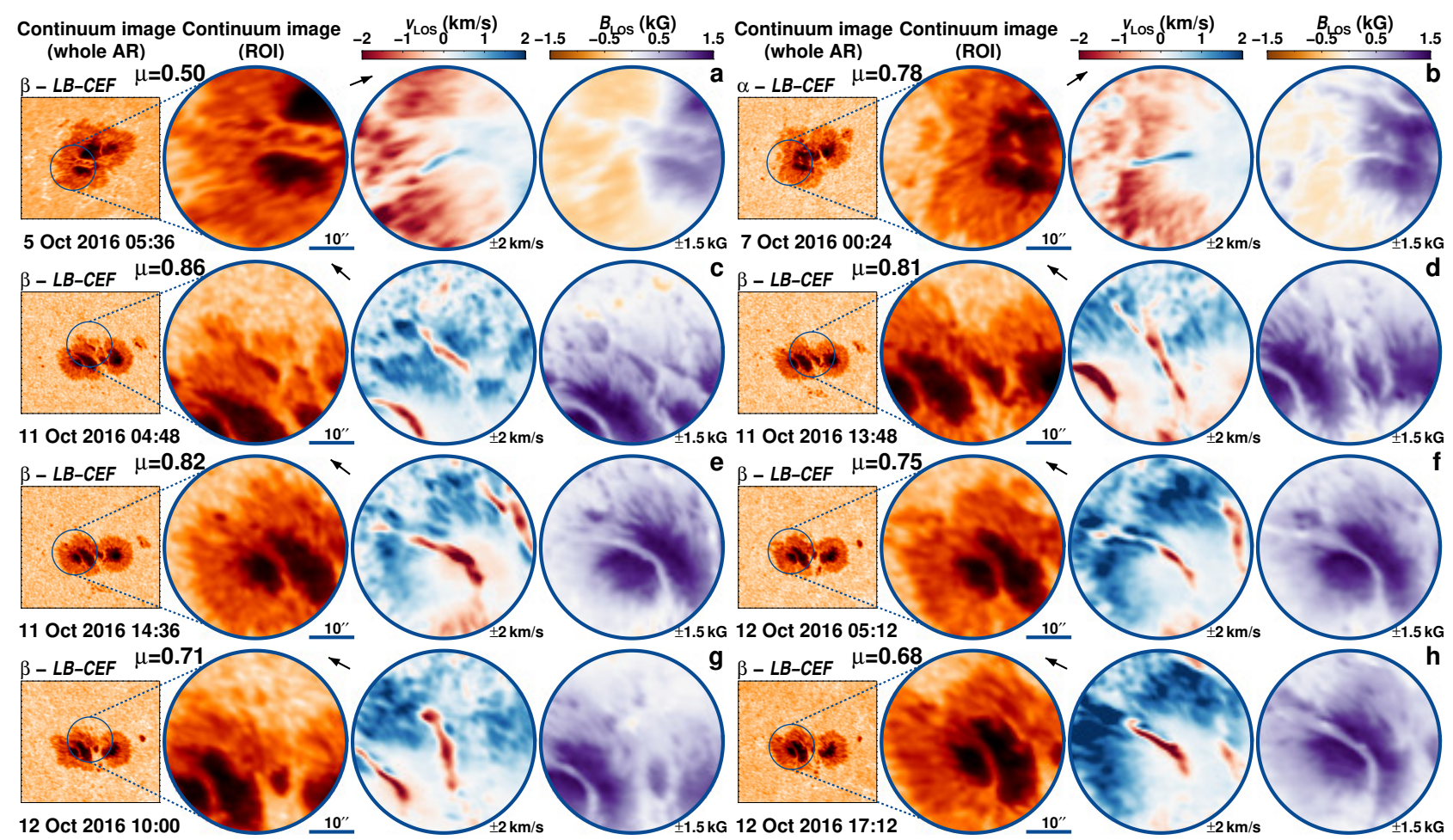

Fig. B.3. AR 12599 followed for 9.2 days from 5-Oct-2016 starting at 00:00 UT.

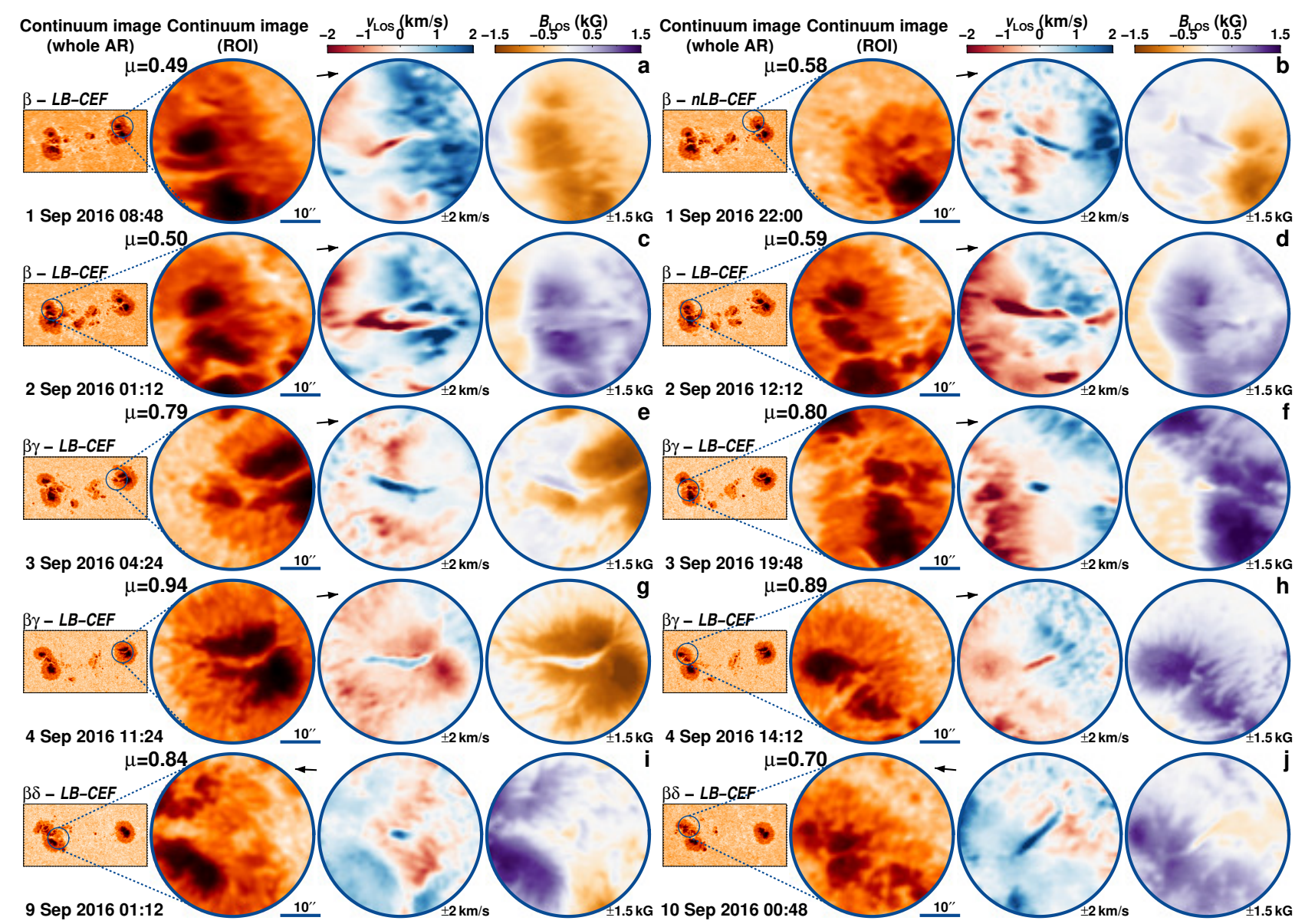

Fig. B.4. AR 12585 followed for 9.4 days from 1-Sep-2016 starting at 00:36 UT. 


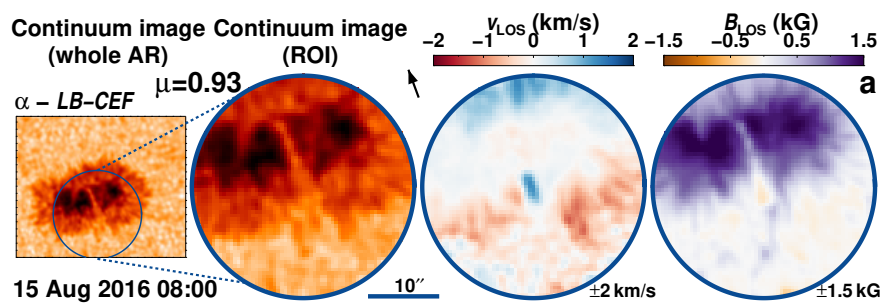

Fig. B.5. AR 12576 followed for 6.4 days from 10-Aug-2016 starting at 14:36 UT.

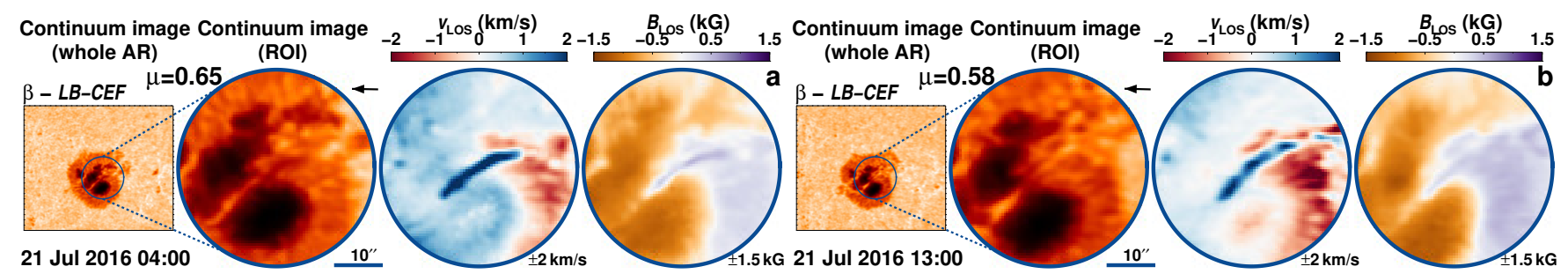

Fig. B.6. AR 12565 followed for 8.7 days from 14-Jul-2016 starting at 17:00 UT.
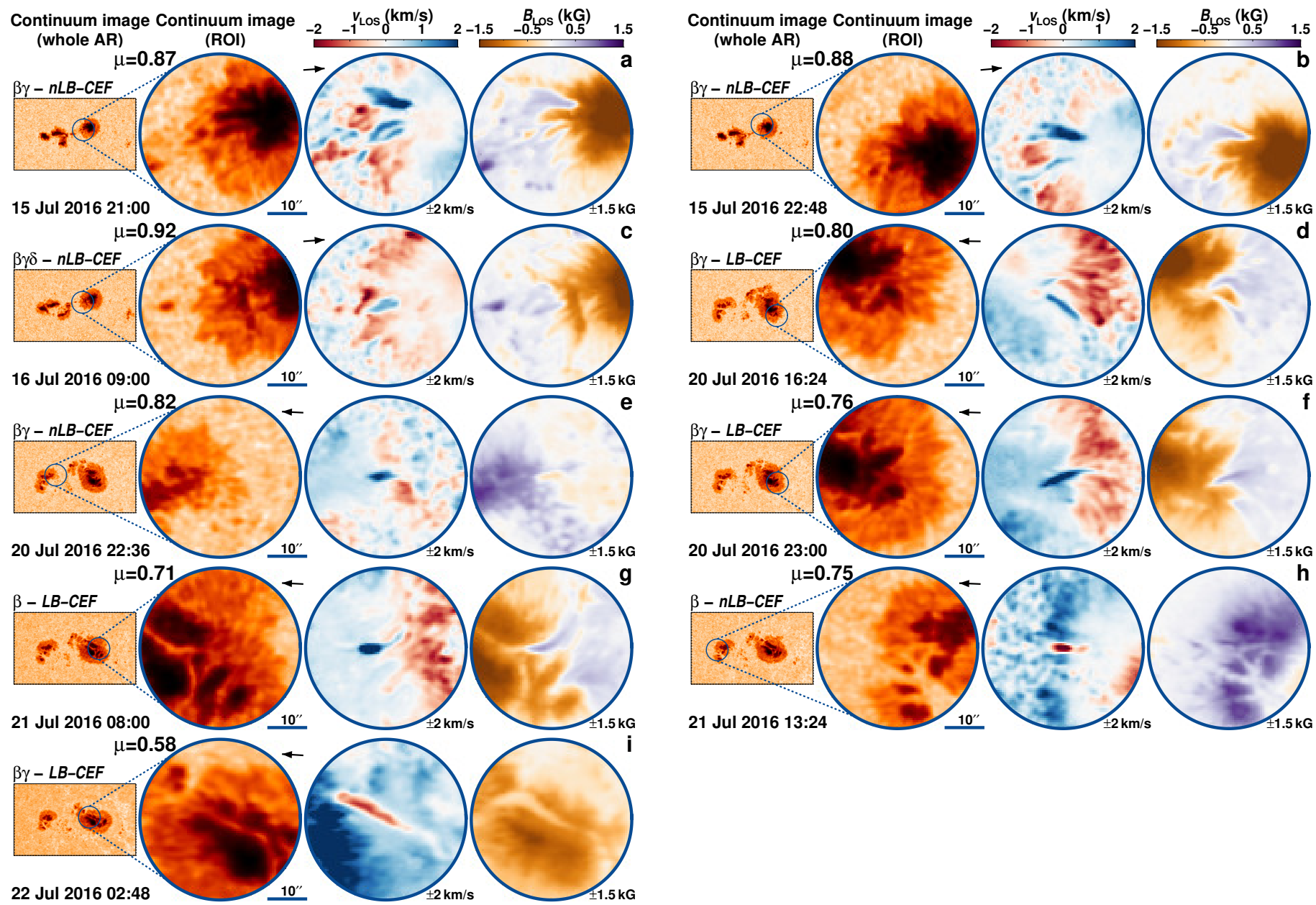

Fig. B.7. AR 12567 followed for 10.6 days from 12-Jul-2016 starting at 19:00 UT. 


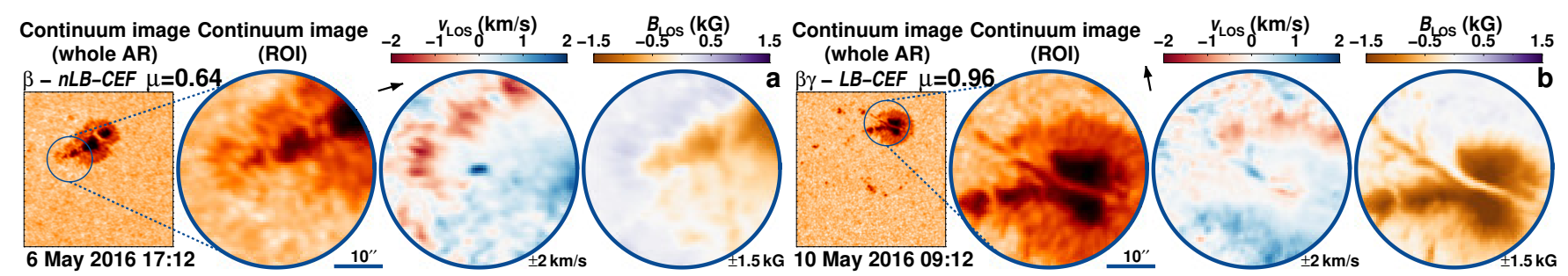

Fig. B.8. AR 12542 followed for 8.1 days from 5-May-2016 starting at 21:00 UT.

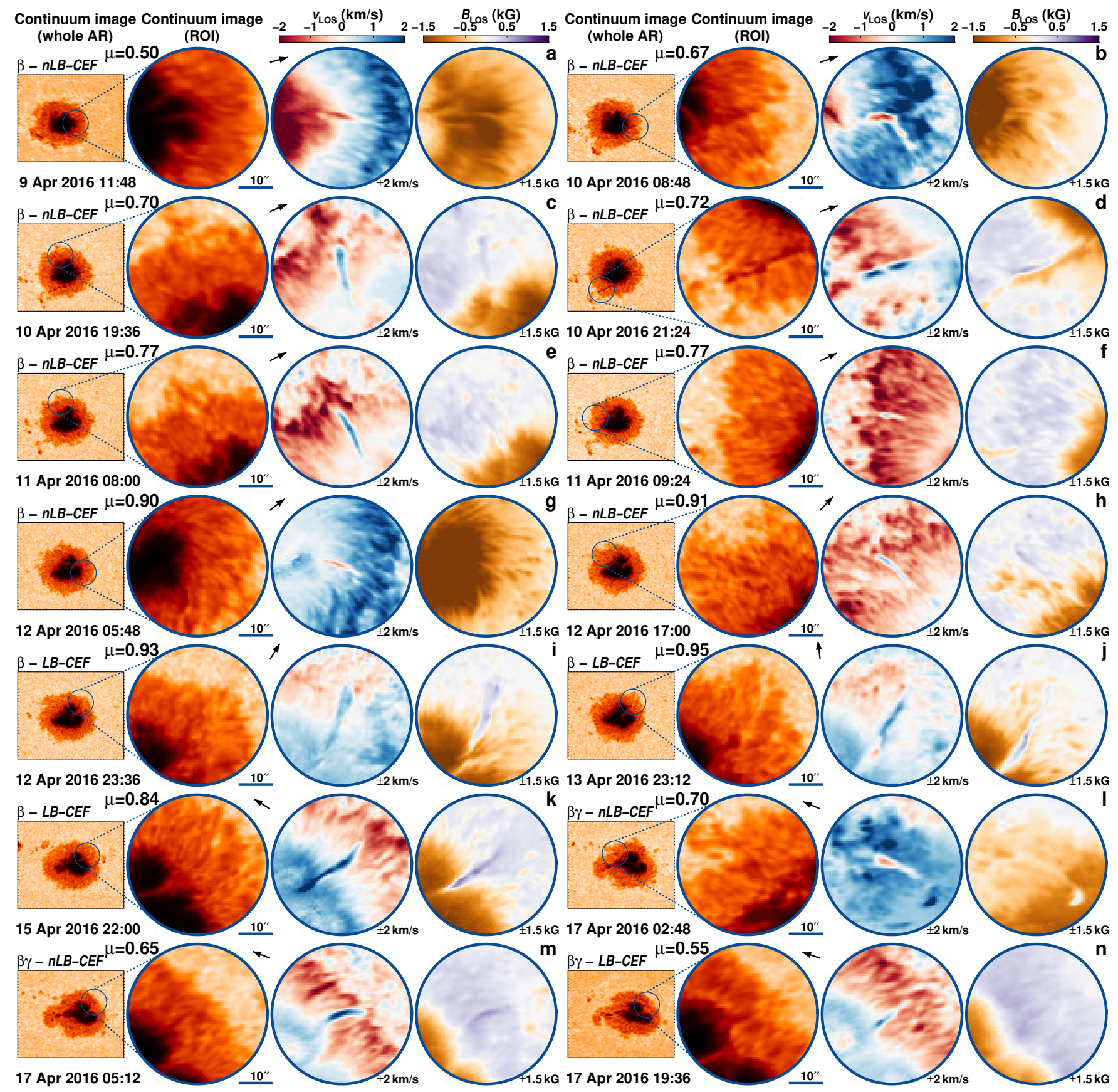

Fig. B.9. AR 12529 followed for 9.2 days from 9-Apr-2016 starting at 08:00 UT. 


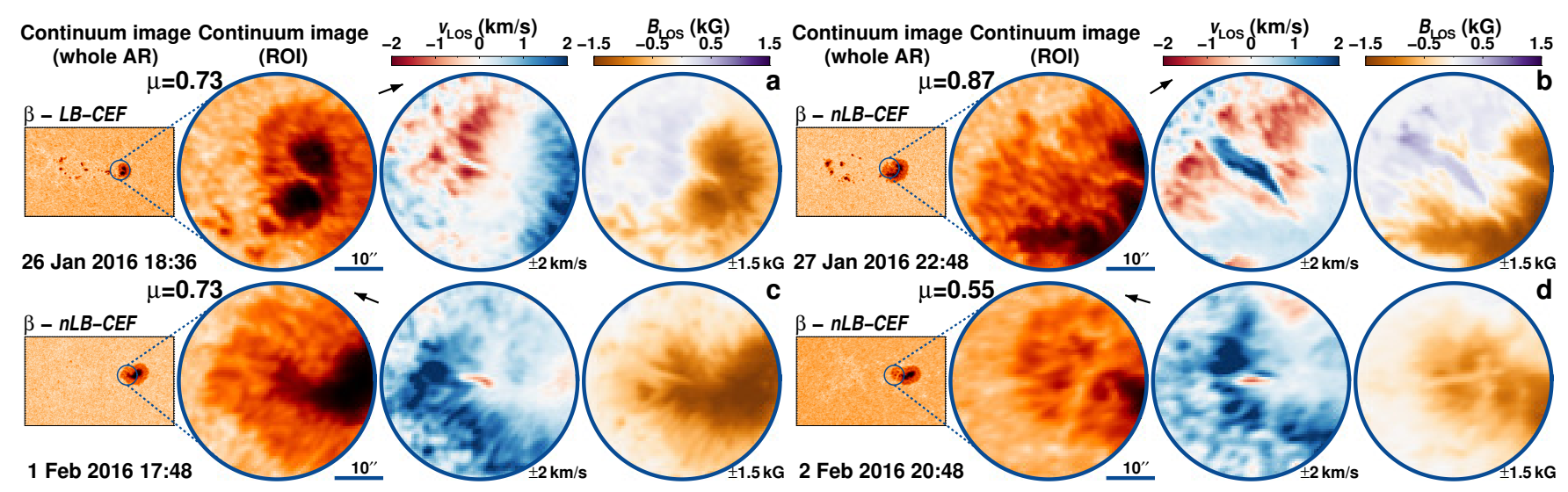

Fig. B.10. AR 12489 followed for 8.9 days from 26-Jan-2016 starting at 00:00 UT.

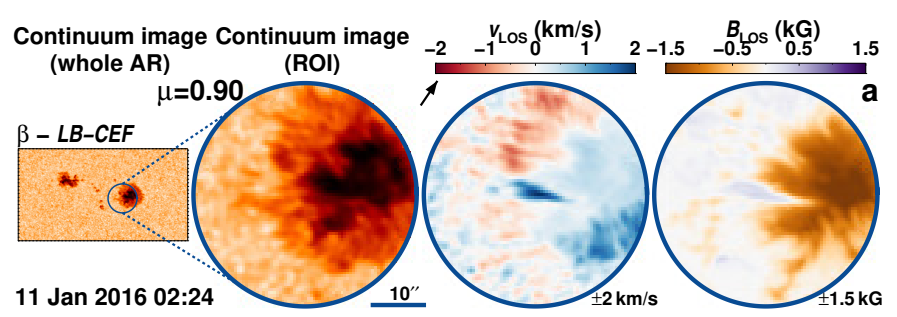

Fig. B.11. AR 12483 followed for 8.6 days from 8-Jan-2016 starting at 10:36 UT.

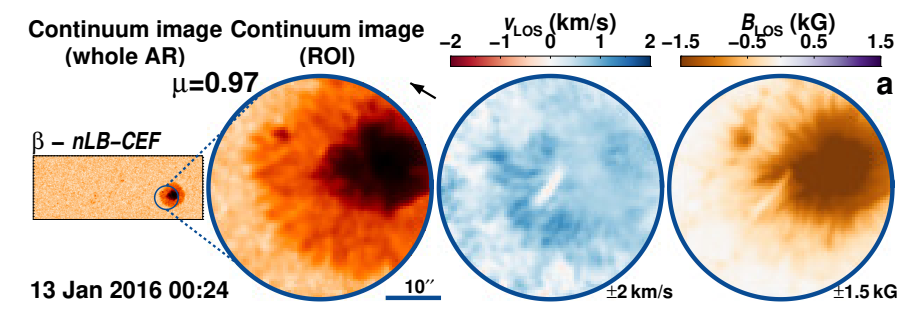

Fig. B.12. AR 12480 followed for 9.4 days from 7-Jan-2016 starting at 13:36 UT.

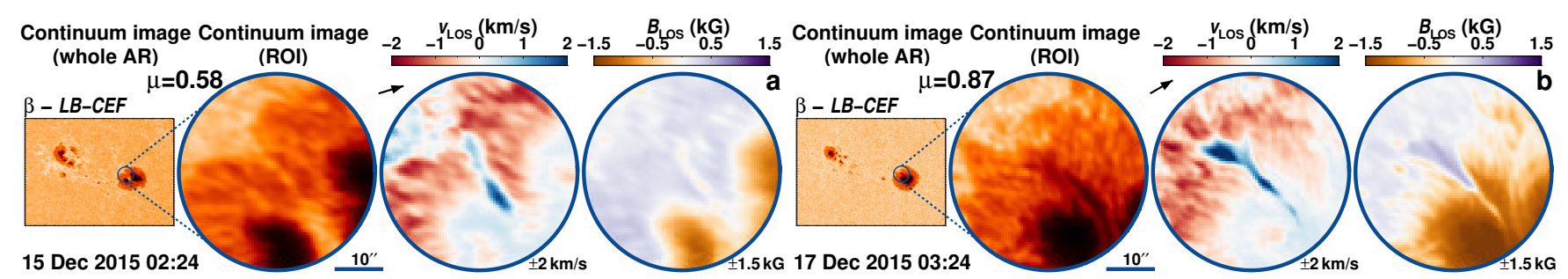

Fig. B.13. AR 12470 followed for 10.9 days from 13-Dec-2015 starting at 21:36 UT. 
J. S. Castellanos Durán et al.: How rare are counter Evershed flows?
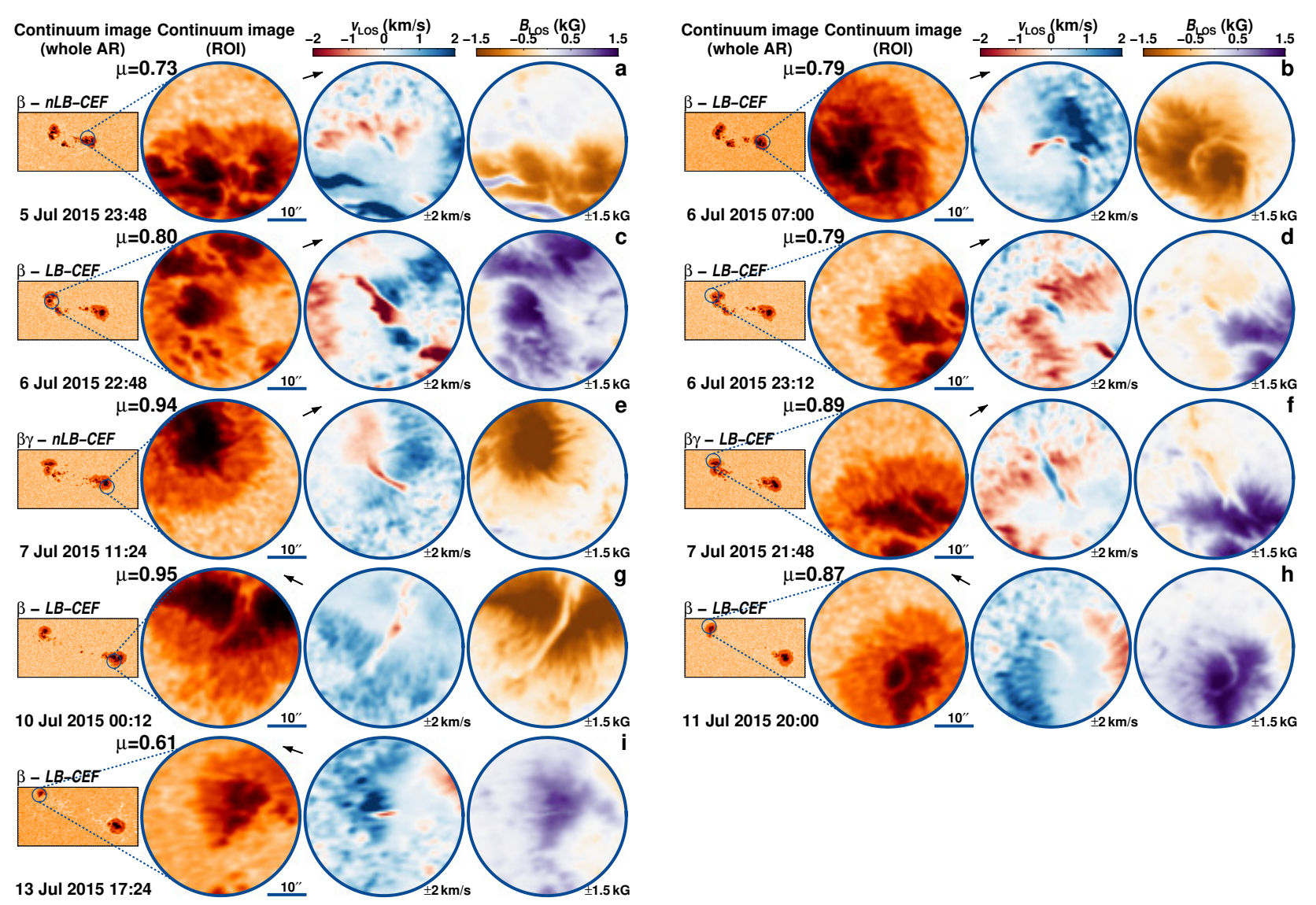

Fig. B.14. AR 12381 followed for 10.7 days from 5-Jul-2015 starting at 06:12 UT.
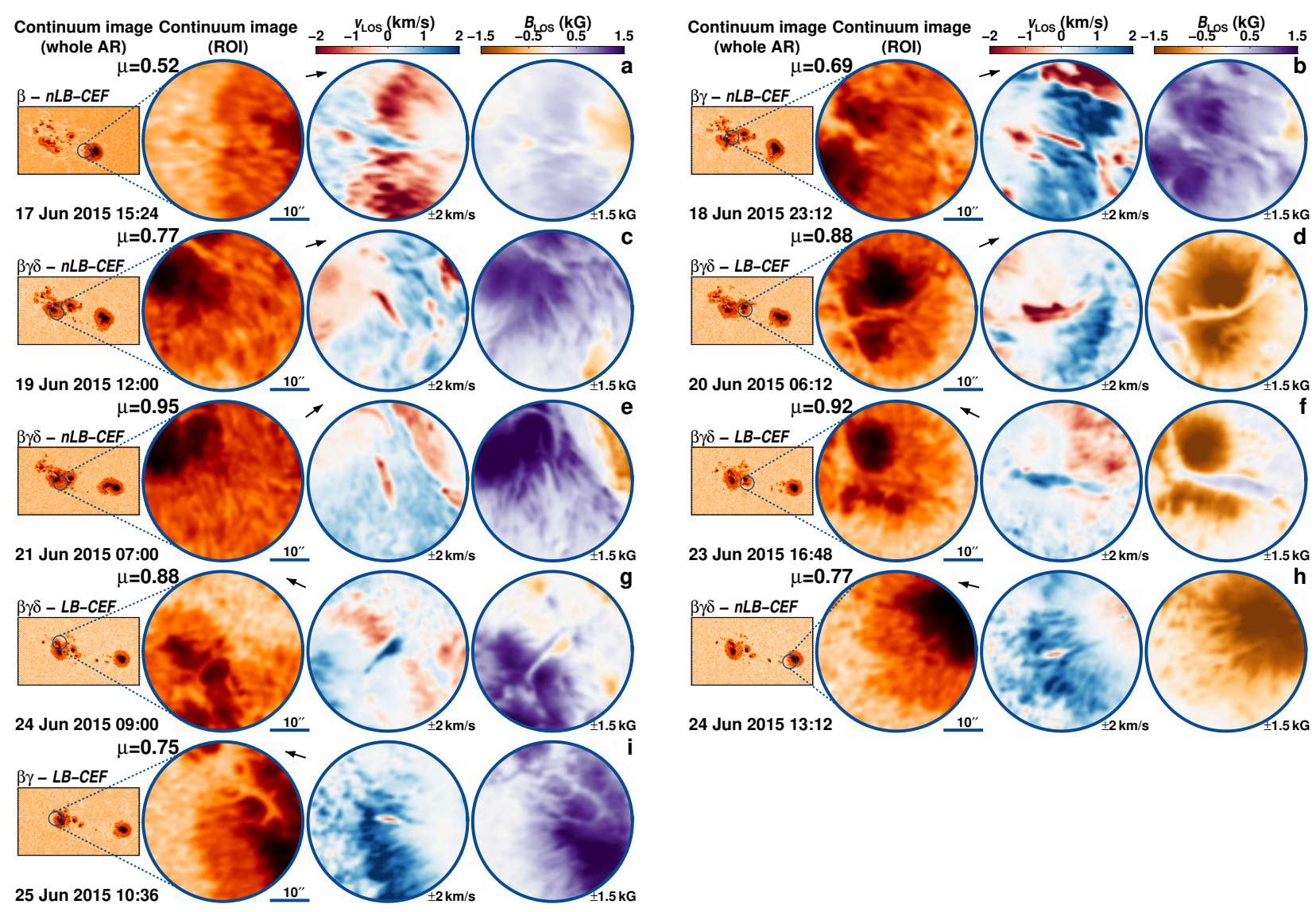

Fig. B.15. AR 12371 followed for 9.6 days from 17-Jun-2015 starting at 05:00 UT. 


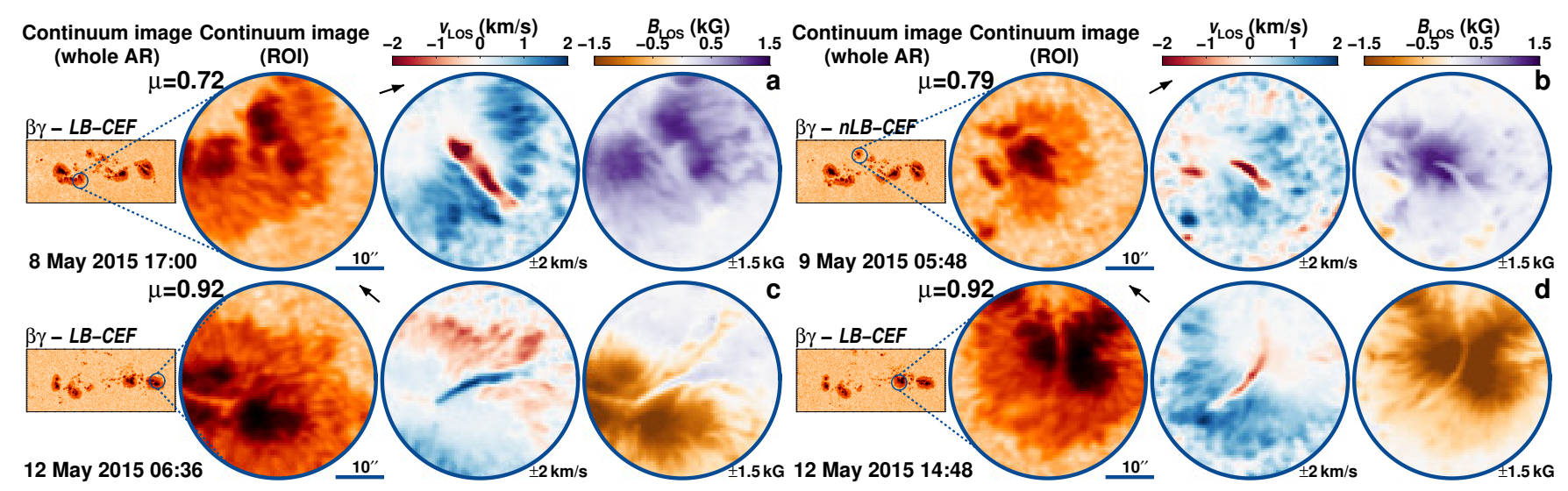

Fig. B.16. AR 12339 followed for 10.3 days from 6-May-2015 starting at 08:12 UT.
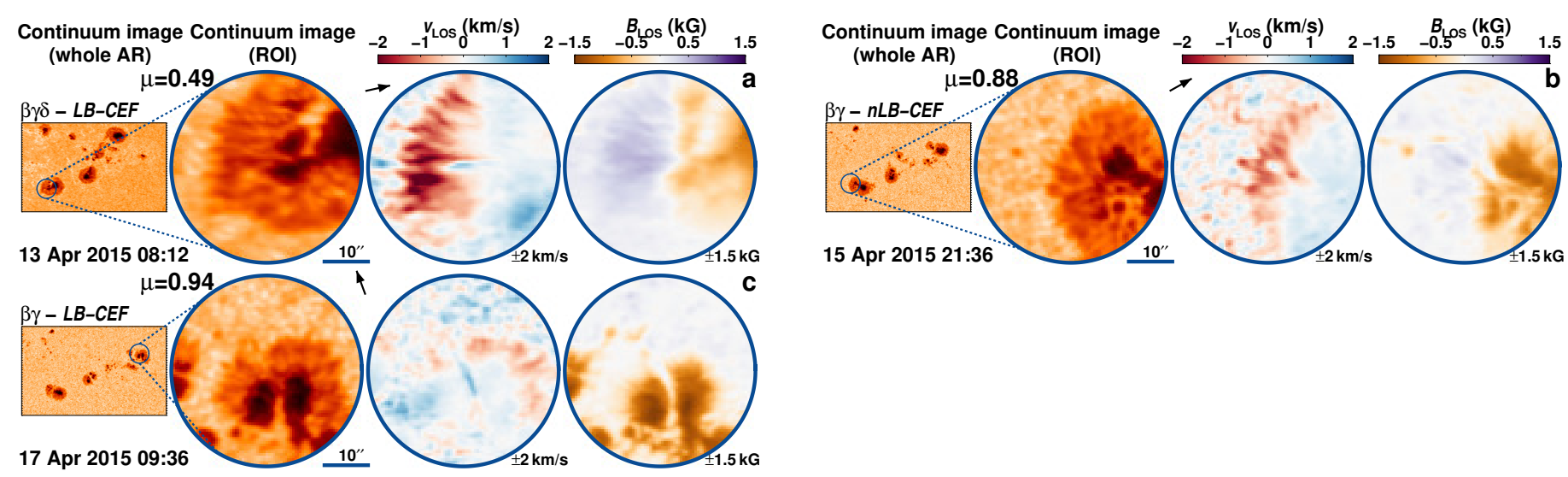

Fig. B.17. AR 12321 followed for 9.1 days from 13-Apr-2015 starting at 05:12 UT.
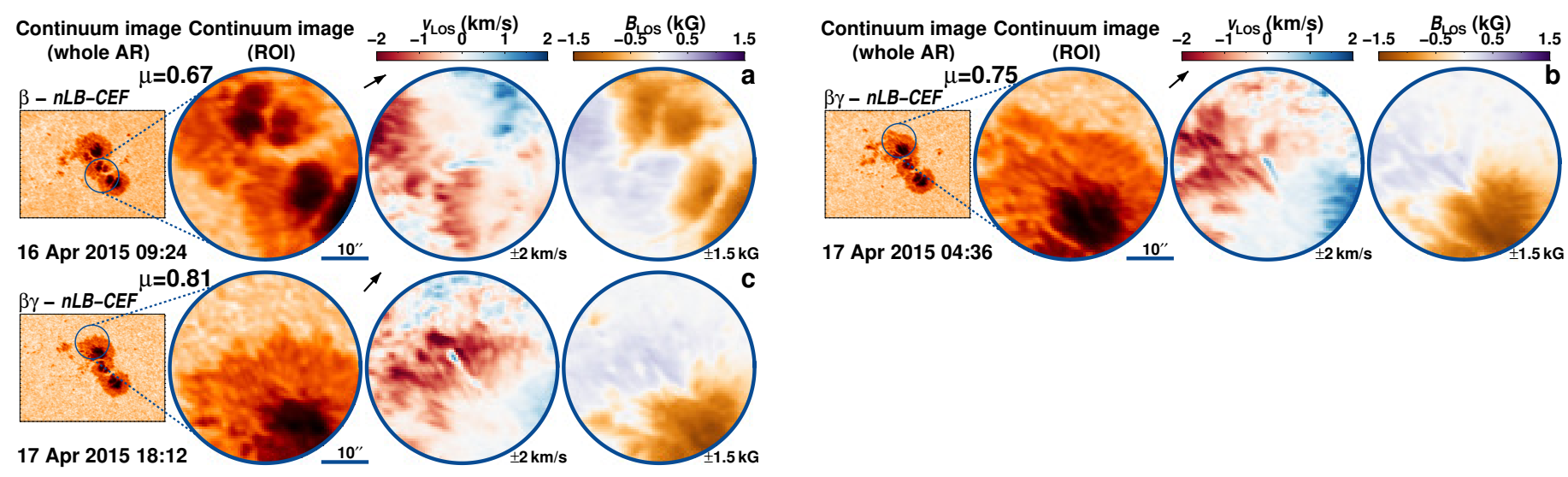

Fig. B.18. AR 12324 followed for 9.0 days from 14-Apr-2015 starting at 23:12 UT. 


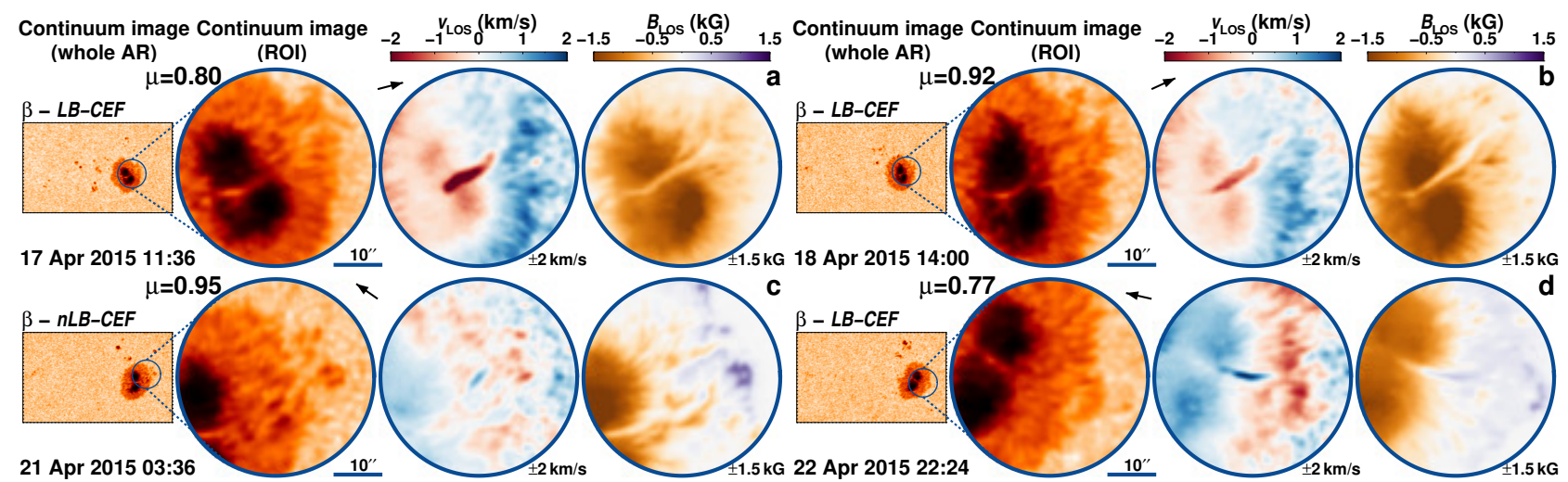

Fig. B.19. AR 12325 followed for 9.4 days from 15-Apr-2015 starting at 06:24 UT.
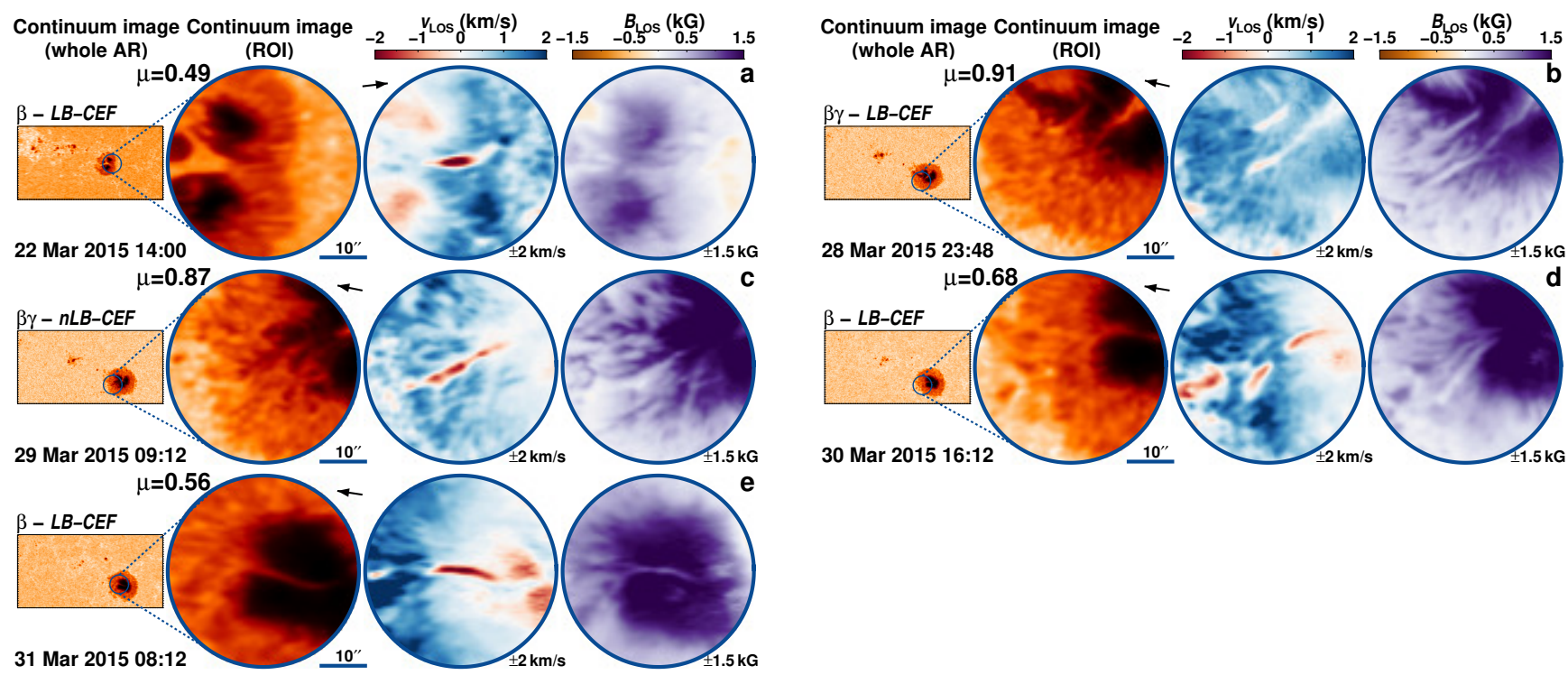

Fig. B.20. AR 12305 followed for 9.9 days from 22-Mar-2015 starting at 07:00 UT.
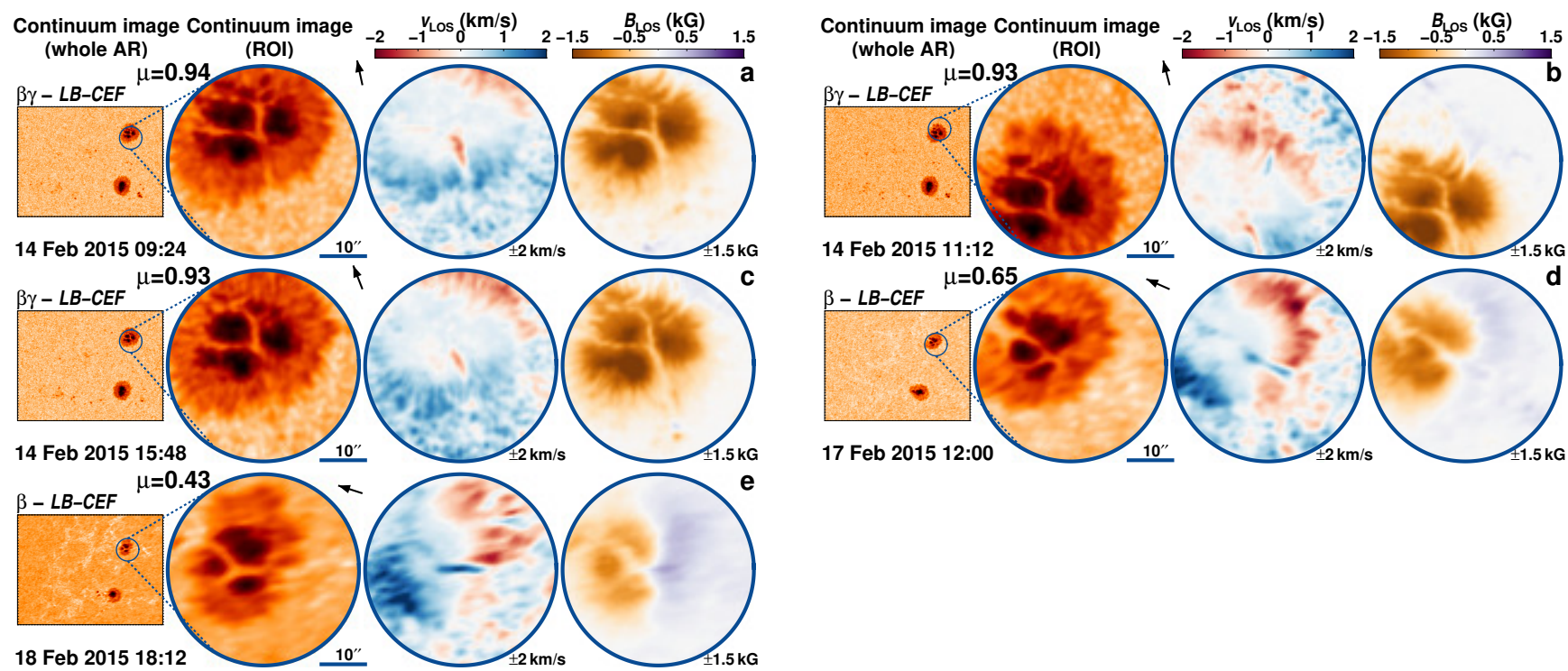

Fig. B.21. AR 12282 followed for 10.5 days from 8-Feb-2015 starting at 23:24 UT. 


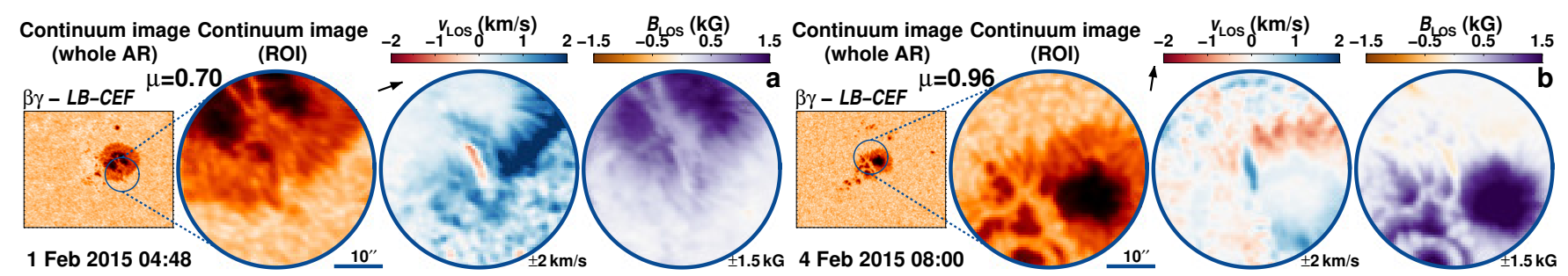

Fig. B.22. AR 12277 followed for 10.2 days from 30-Jan-2015 starting at 02:48 UT.

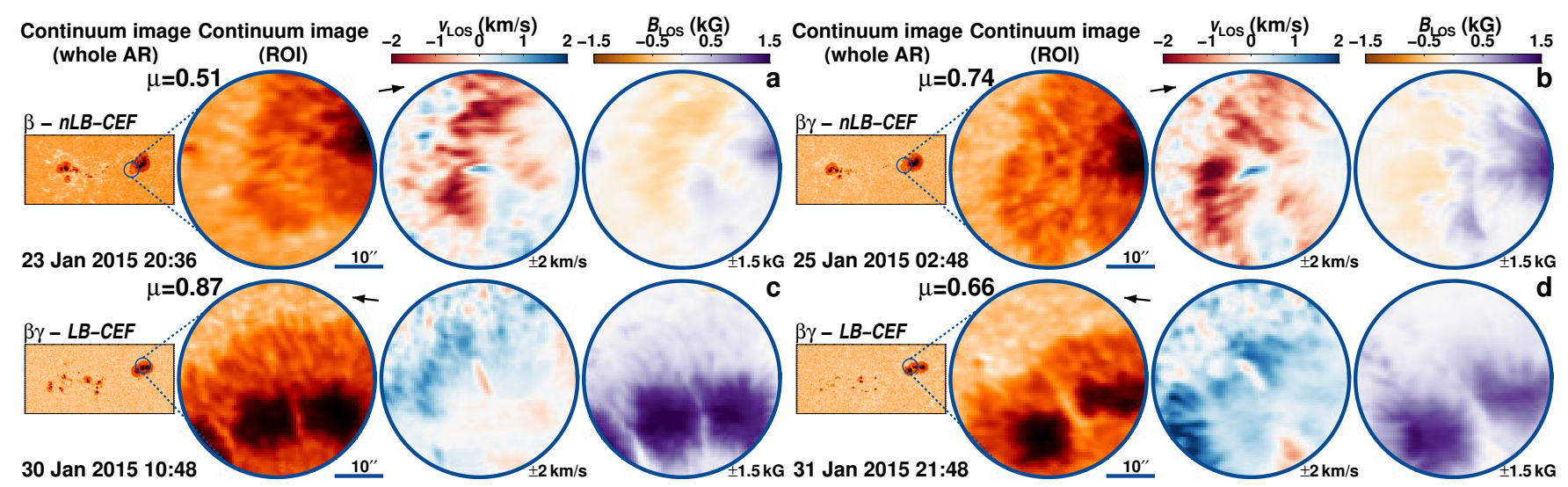

Fig. B.23. AR 12268 followed for 10.6 days from 22-Jan-2015 starting at 21:48 UT.

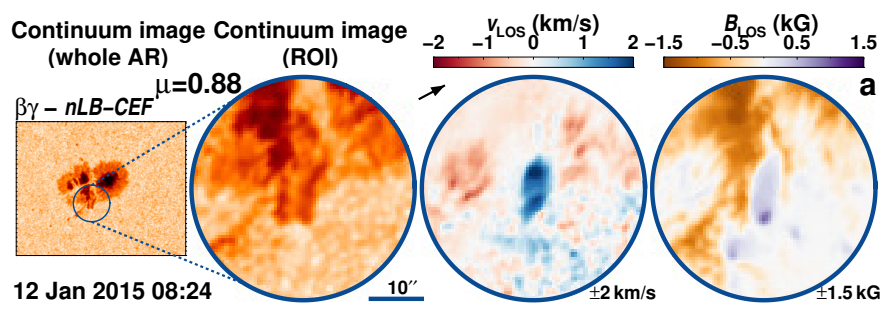

Fig. B.24. AR 12259 followed for 11.6 days from 8-Jan-2015 starting at 13:24 UT.

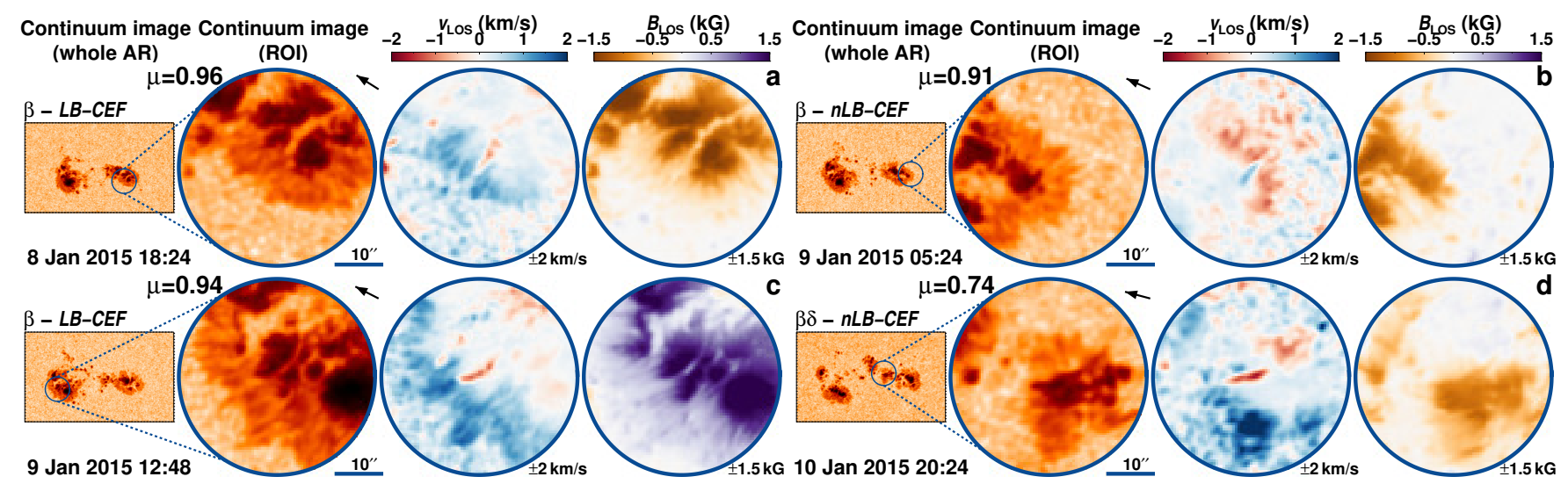

Fig. B.25. AR 12257 followed for 6.8 days from 6-Jan-2015 starting at 13:36 UT. 
J. S. Castellanos Durán et al.: How rare are counter Evershed flows?
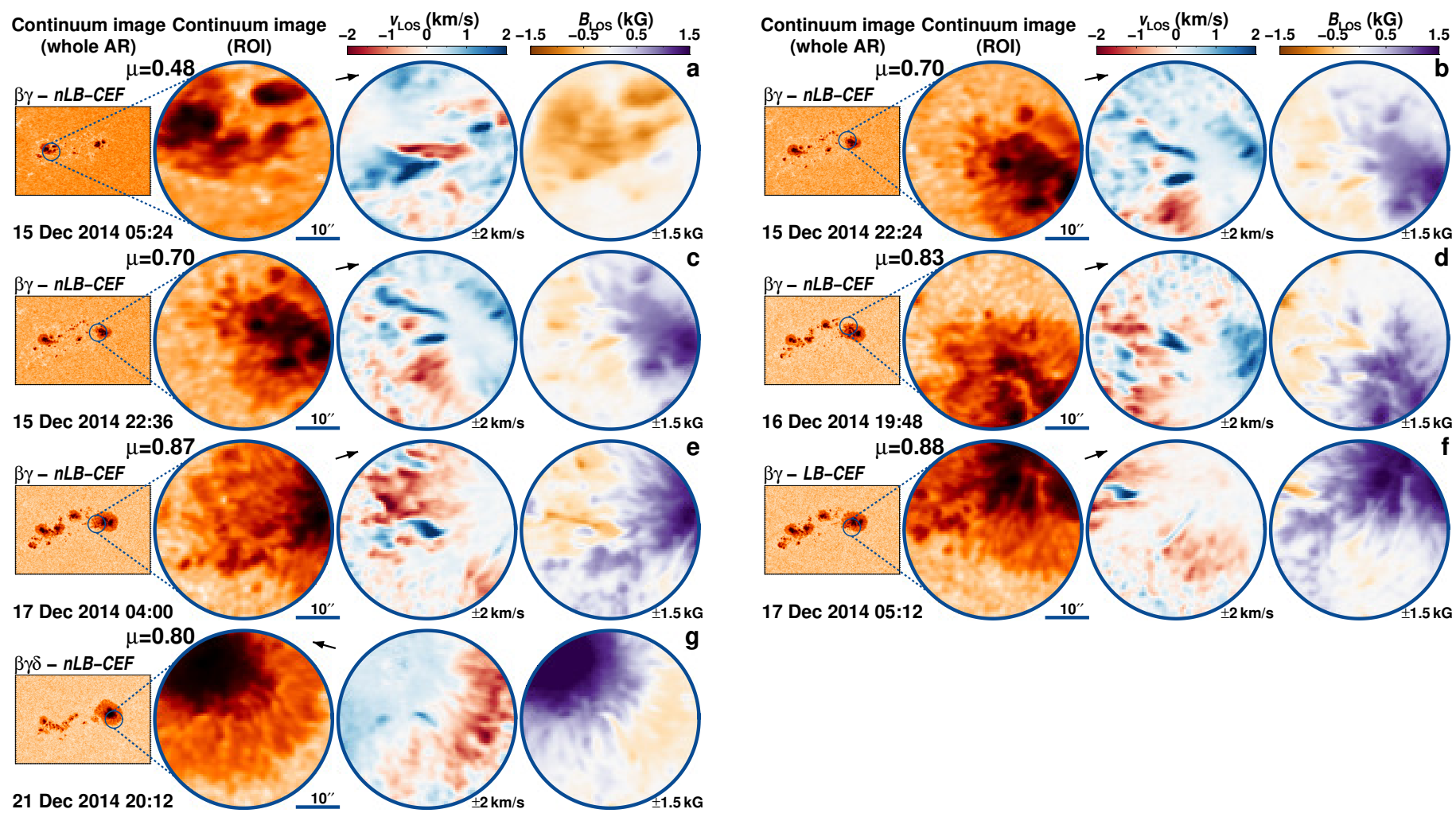

Fig. B.26. AR 12241 followed for 8.8 days from 15-Dec-2014 starting at 03:12 UT.
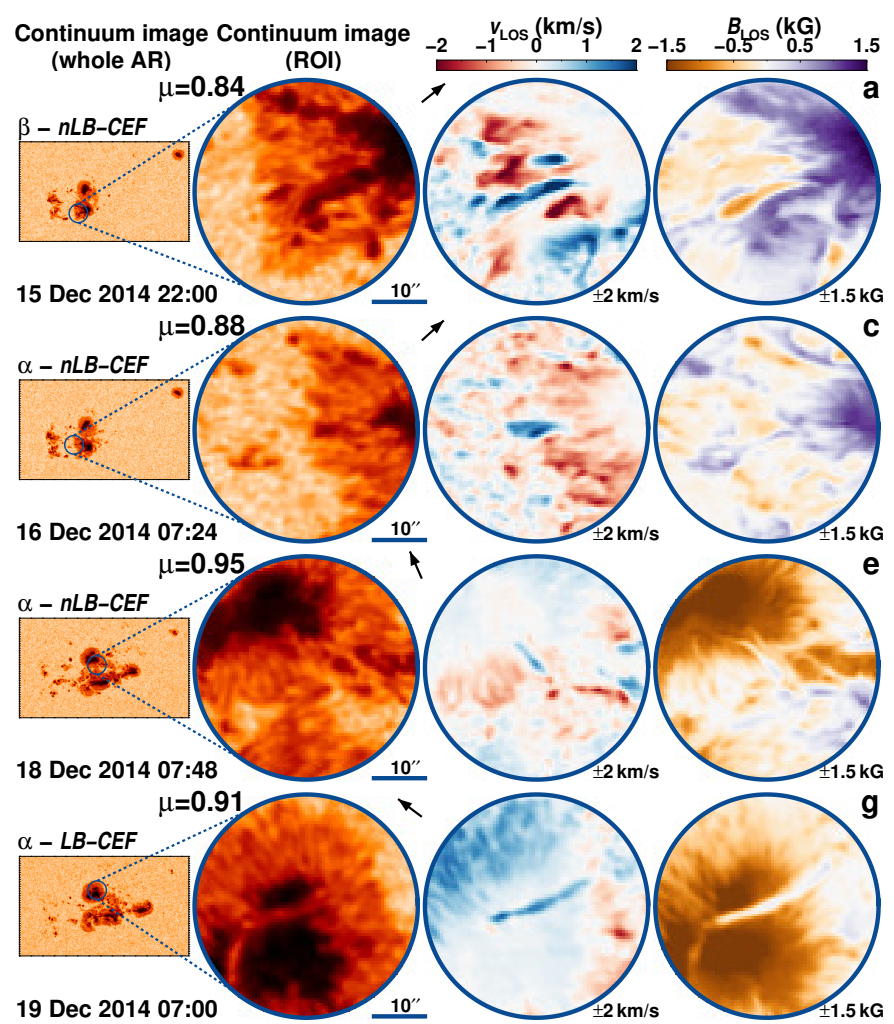
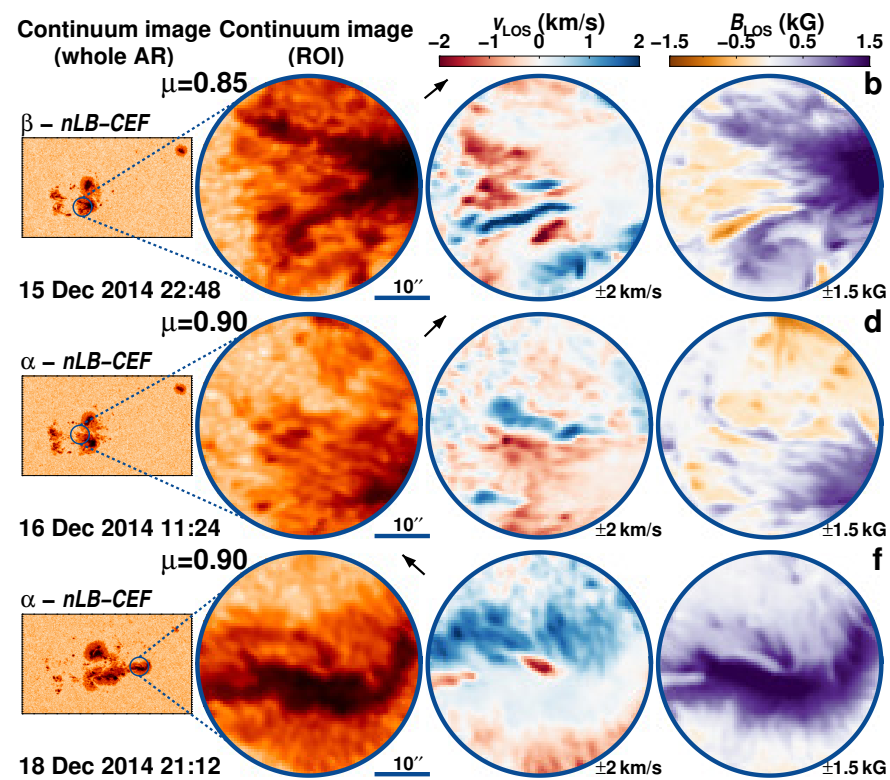
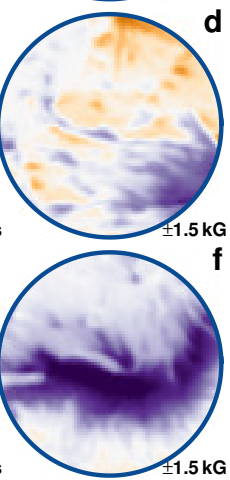

Fig. B.27. AR 12237 followed for 8.2 days from 13-Dec-2014 starting at 23:00 UT. 


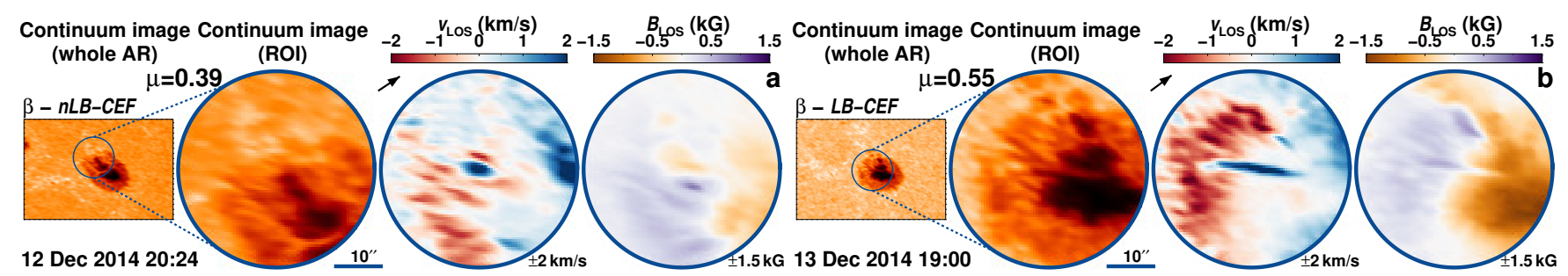

Fig. B.28. AR 12236 followed for 10.0 days from 12-Dec-2014 starting at 02:00 UT.

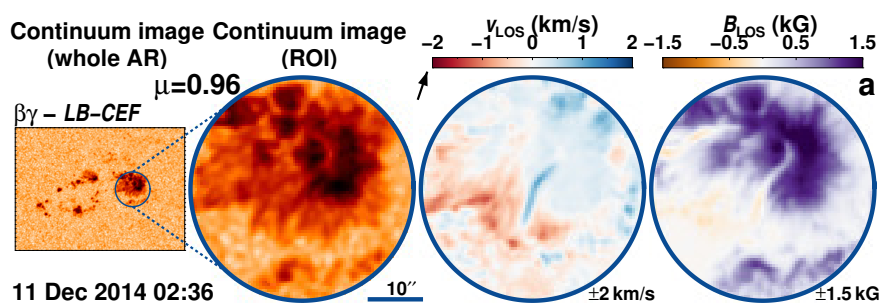

Fig. B.29. AR 12230 followed for 7.4 days from 8-Dec-2014 starting at 15:00 UT.

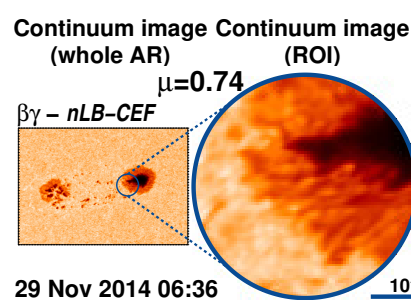

$$
\begin{gathered}
\mu=0.87 \\
\beta \gamma-n L B-C E F \\
4
\end{gathered}
$$

\section{Nov 2014 05:24}

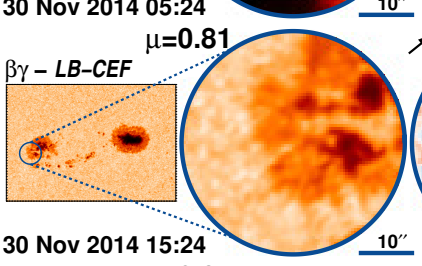

30 Nov 2014 15:24

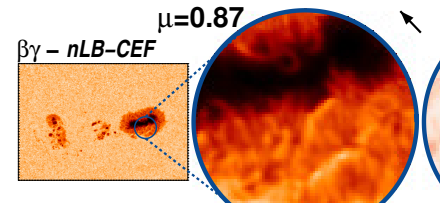

3 Dec 2014 14:12

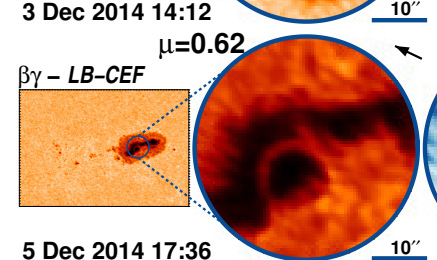

5 Dec 2014 17:36

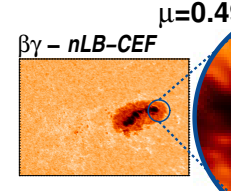

6 Dec 2014 07:24
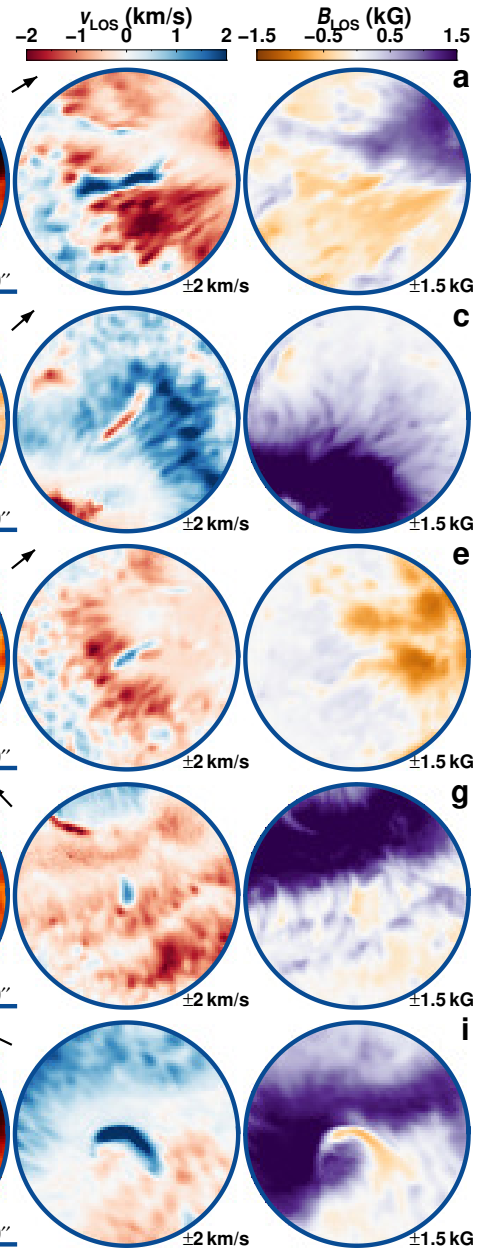

$1.5 \mathrm{kG}$

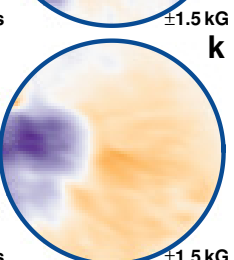

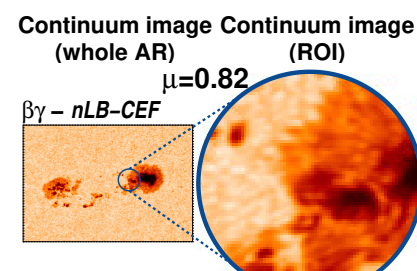

29 Nov 2014 23:24 10"
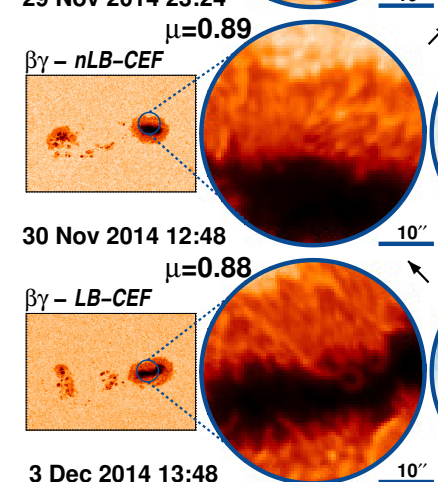

3 Dec 2014 13:48

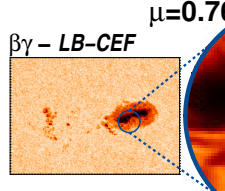

4 Dec 2014 17.24

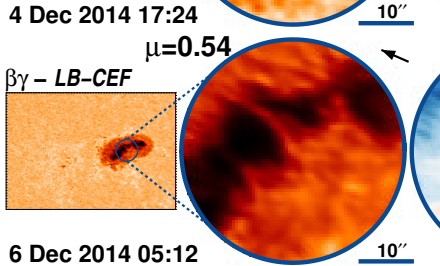

6 Dec 2014 05:12
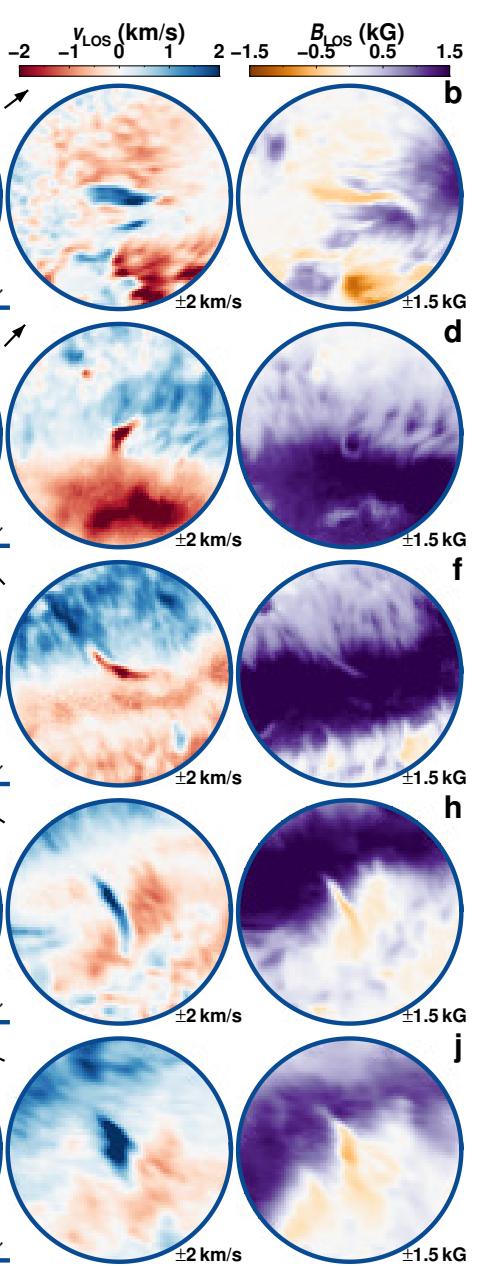

Fig. B.30. AR 12222 followed for 8.8 days from 27-Nov-2014 starting at 20:00 UT. 
J. S. Castellanos Durán et al.: How rare are counter Evershed flows?

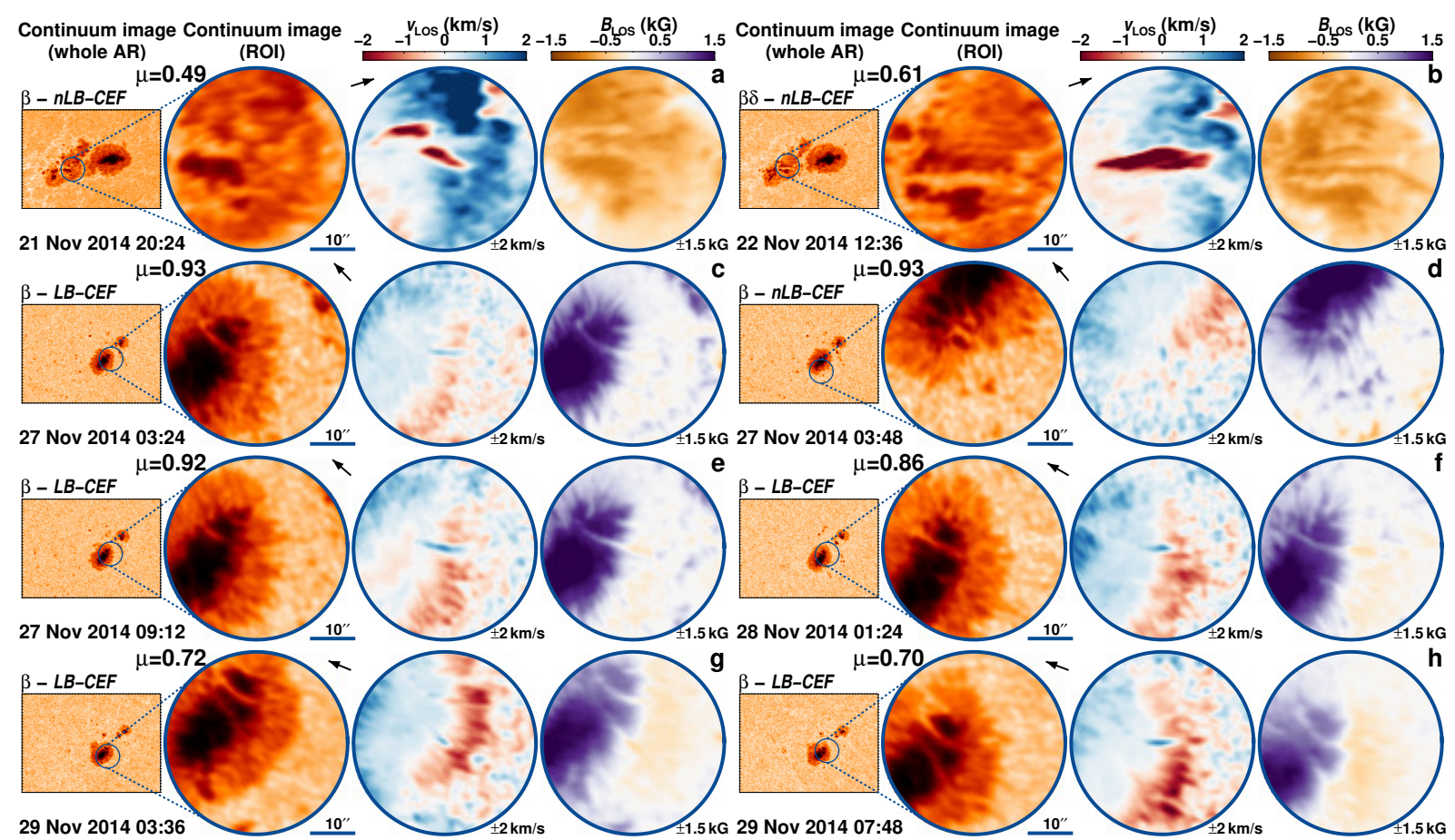

Fig. B.31. AR 12216 followed for 9.6 days from 21-Nov-2014 starting at 00:24 UT.

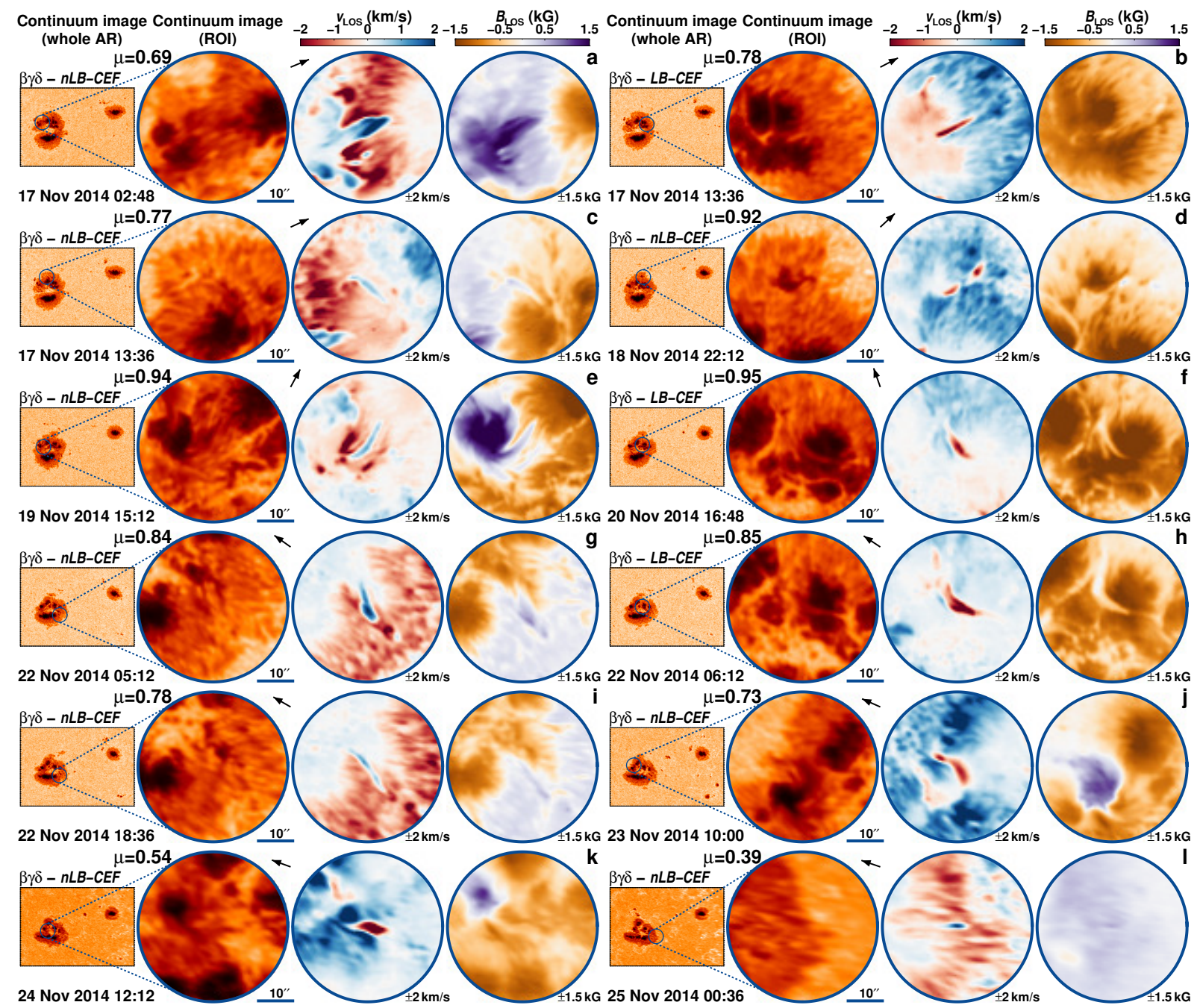

Fig. B.32. AR 12209 followed for 9.9 days from 15-Nov-2014 starting at 18:12 UT. 


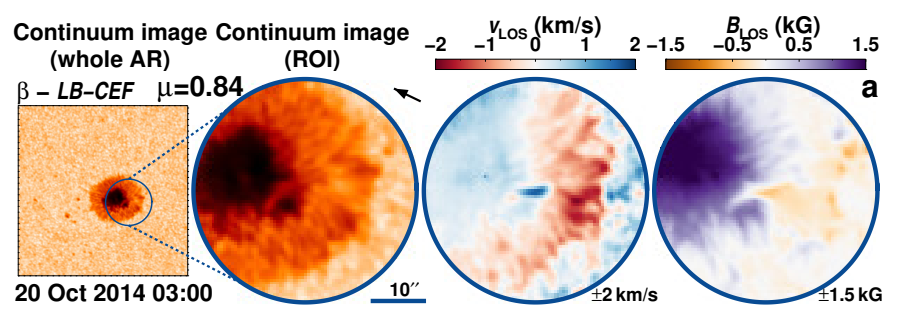

Fig. B.33. AR 12187 followed for 10.2 days from 12-Oct-2014 starting at 21:00 UT.

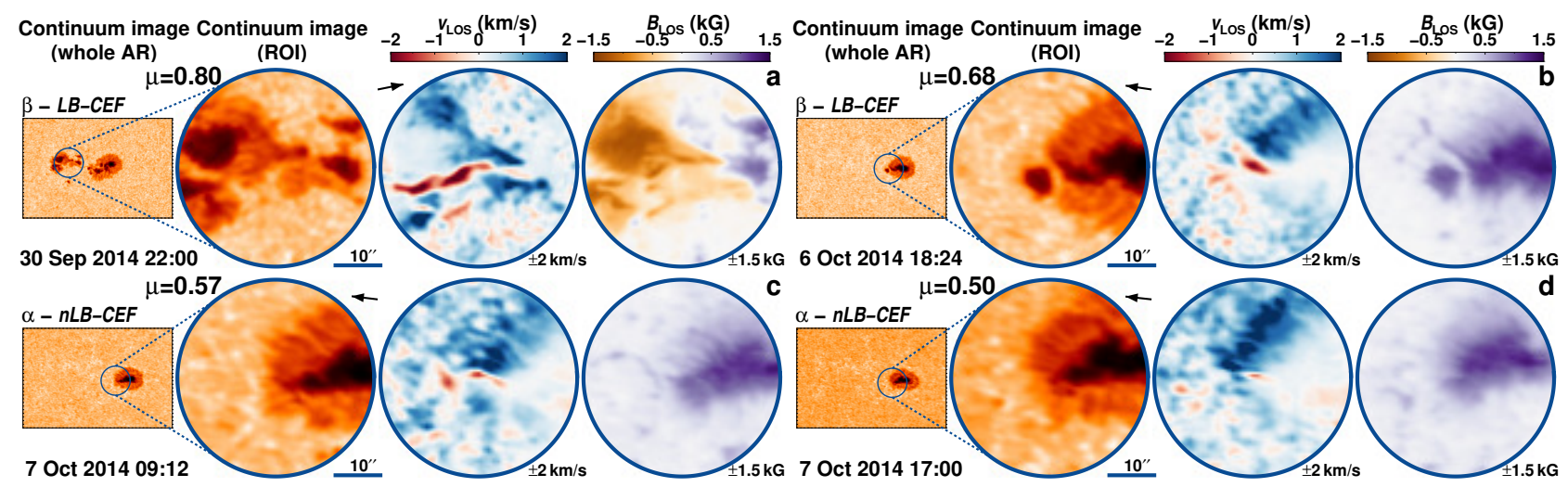

Fig. B.34. AR 12178 followed for 10.4 days from 28-Sep-2014 starting at 02:36 UT.

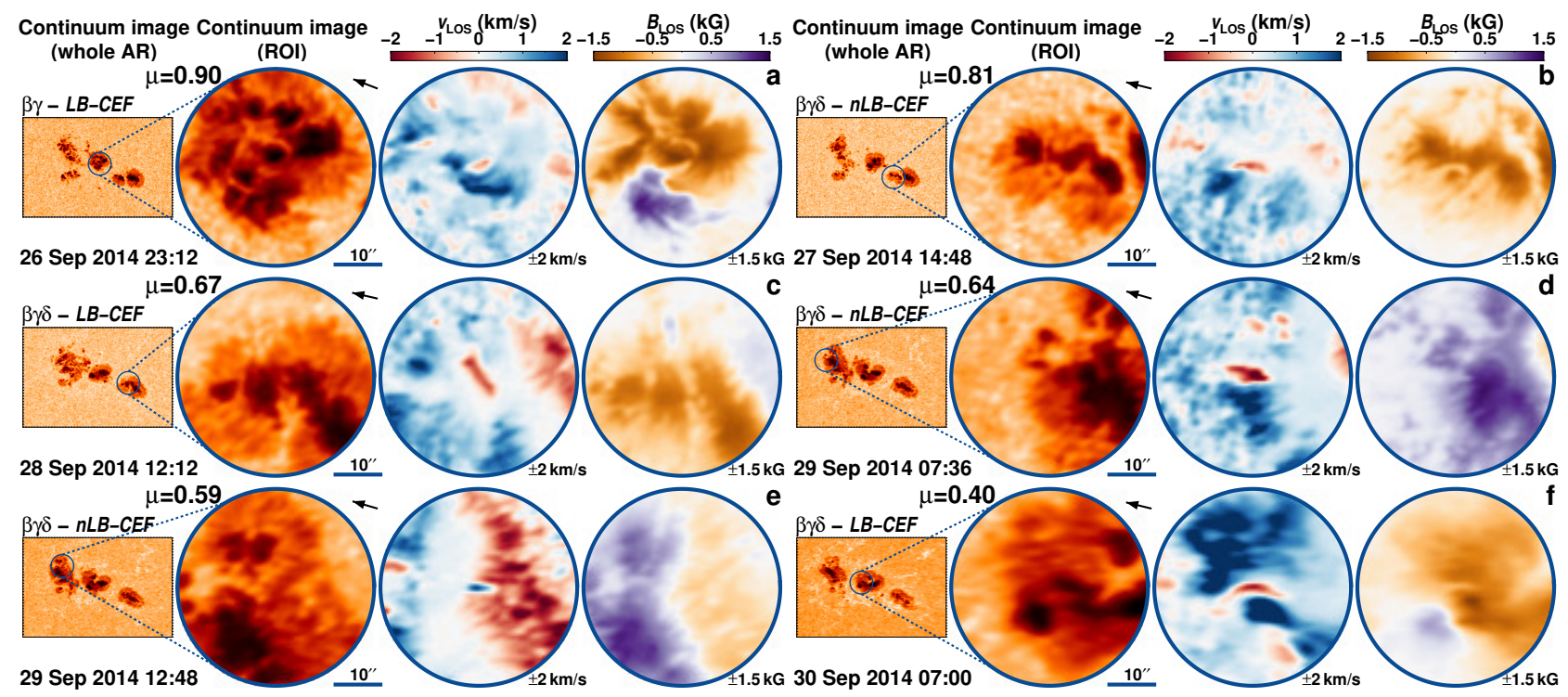

Fig. B.35. AR 12175 followed for 6.2 days from 24-Sep-2014 starting at 19:48 UT.

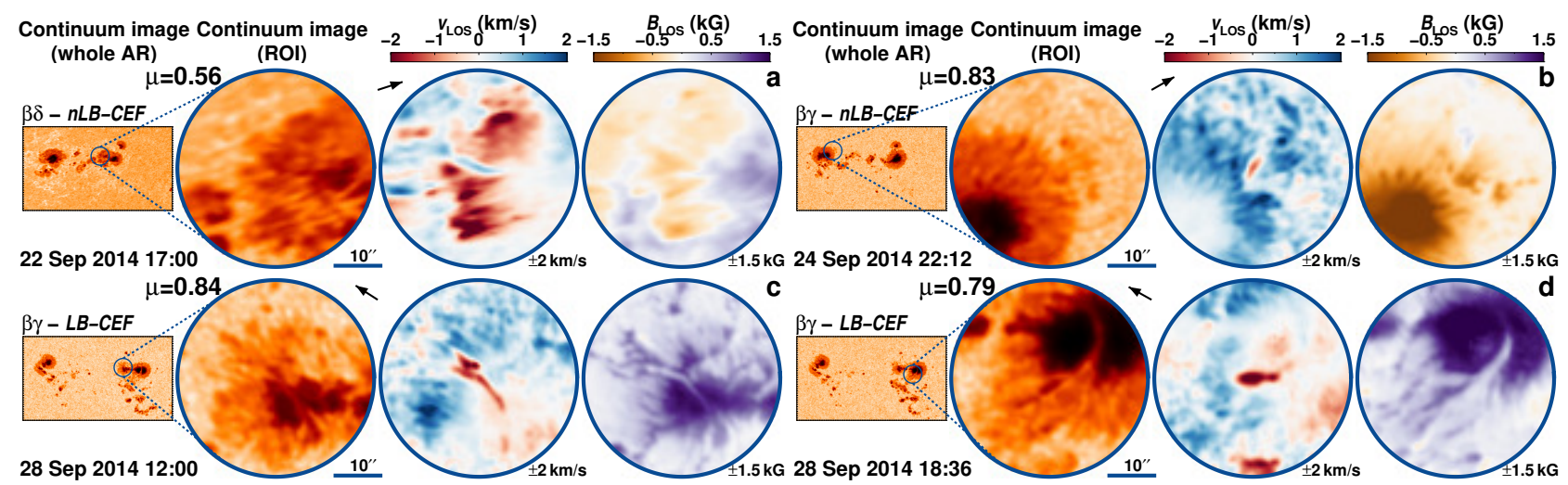

Fig. B.36. AR 12172 followed for 8.3 days from 22-Sep-2014 starting at 12:00 UT. 

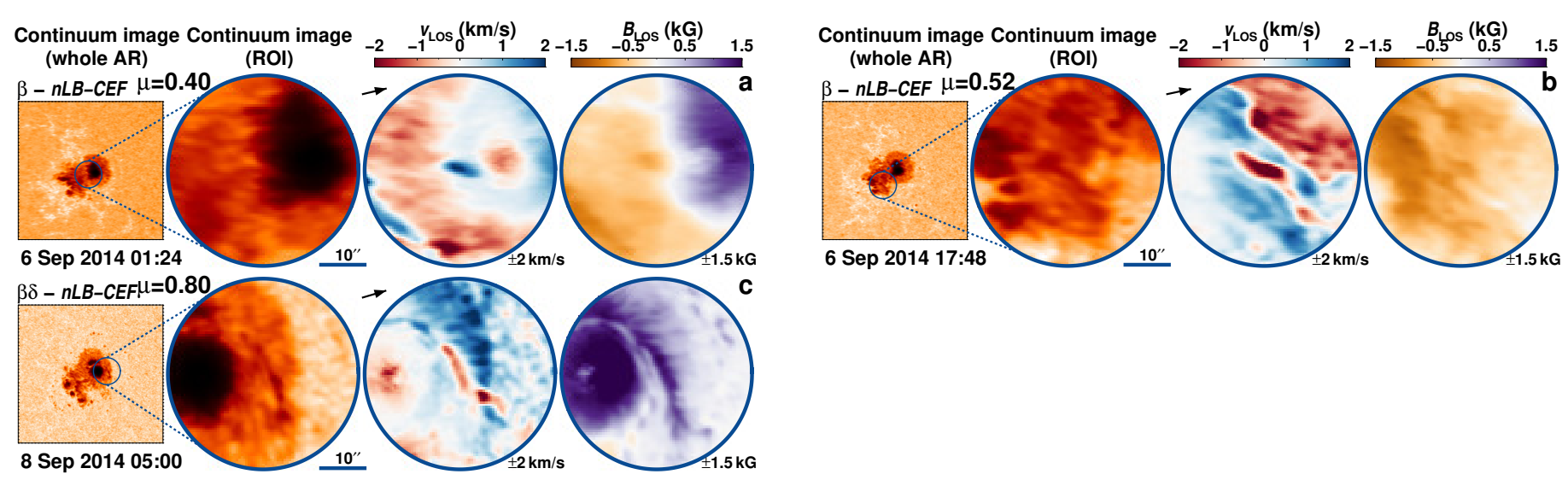

Fig. B.37. AR 12158 followed for 10.6 days from 5-Sep-2014 starting at 18:36 UT.

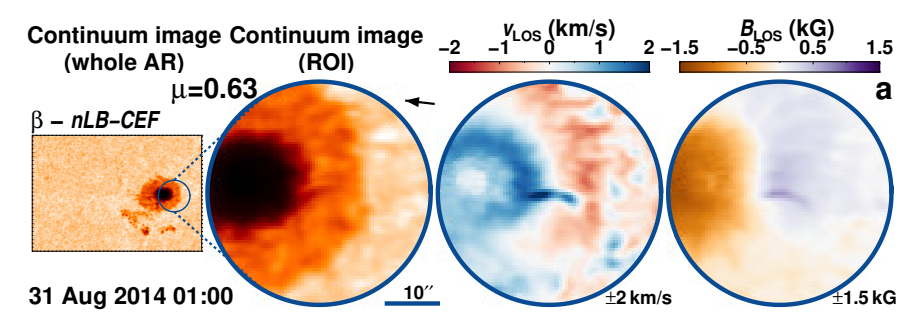

Fig. B.38. AR 12149 followed for 10.5 days from 22-Aug-2014 starting at 07:12 UT.

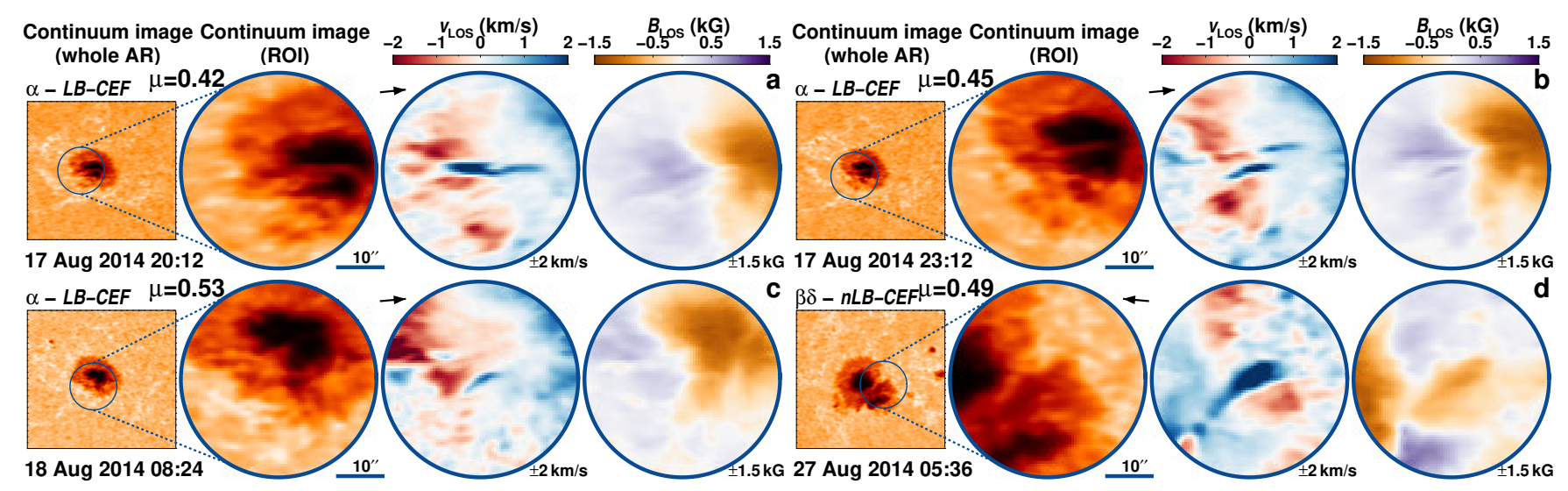

Fig. B.39. AR 12146 followed for 10.6 days from 17-Aug-2014 starting at 12:00 UT.

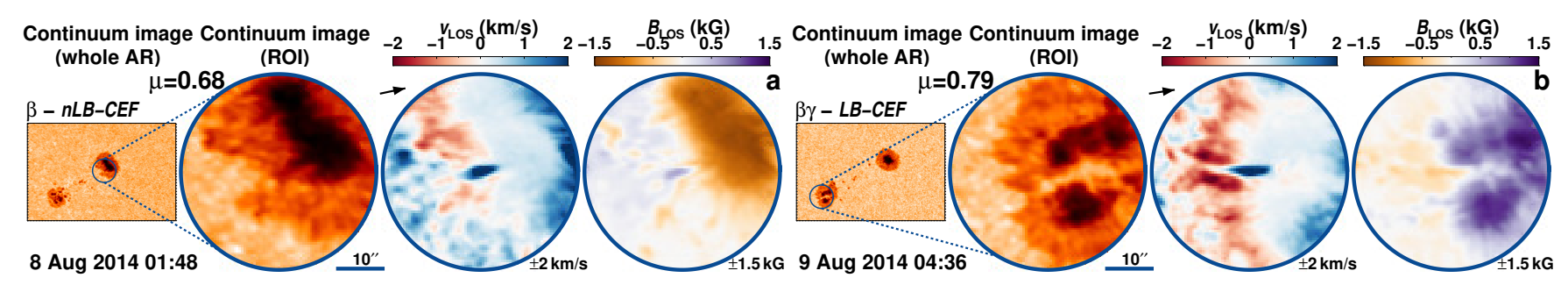

Fig. B.40. AR 12135 followed for 10.4 days from 6-Aug-2014 starting at 10:48 UT. 

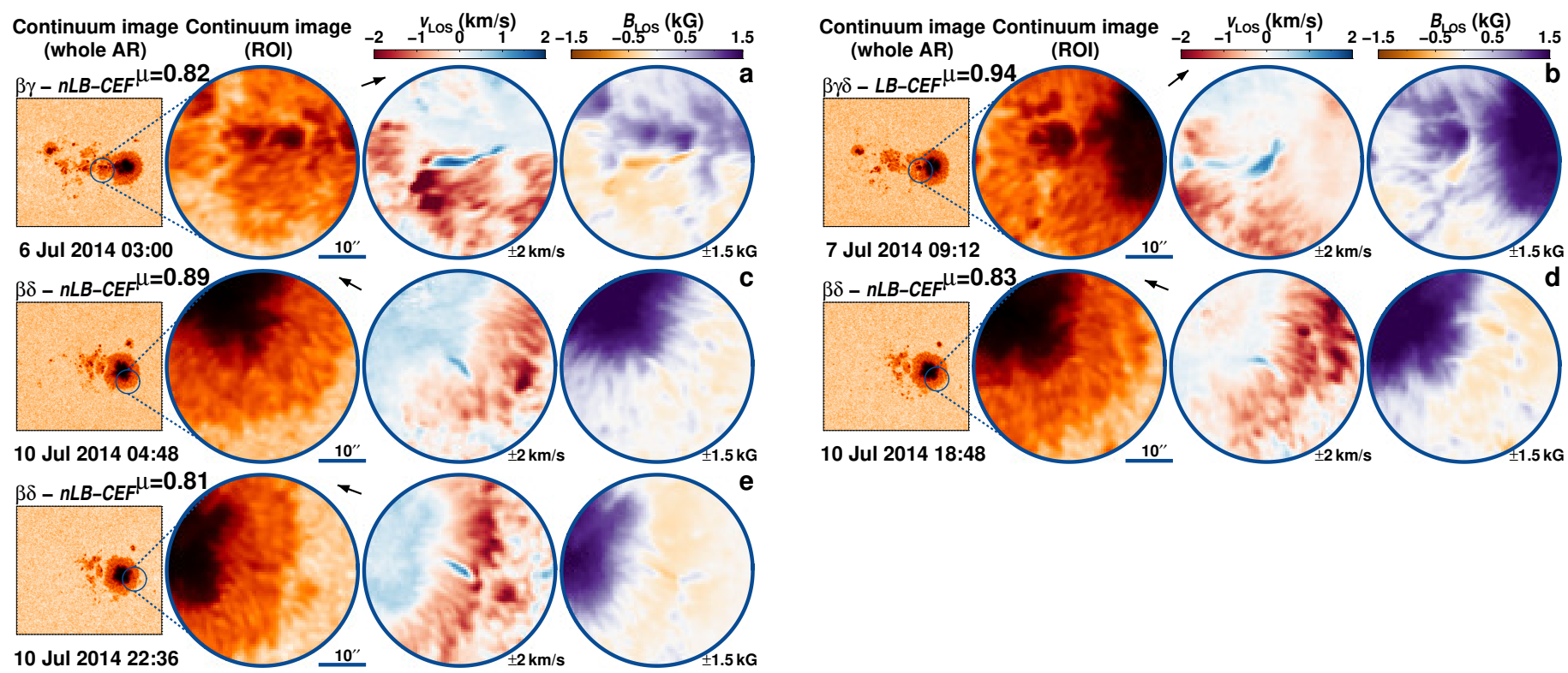

Fig. B.41. AR 12109 followed for 11.1 days from 3-Jul-2014 starting at 03:24 UT.
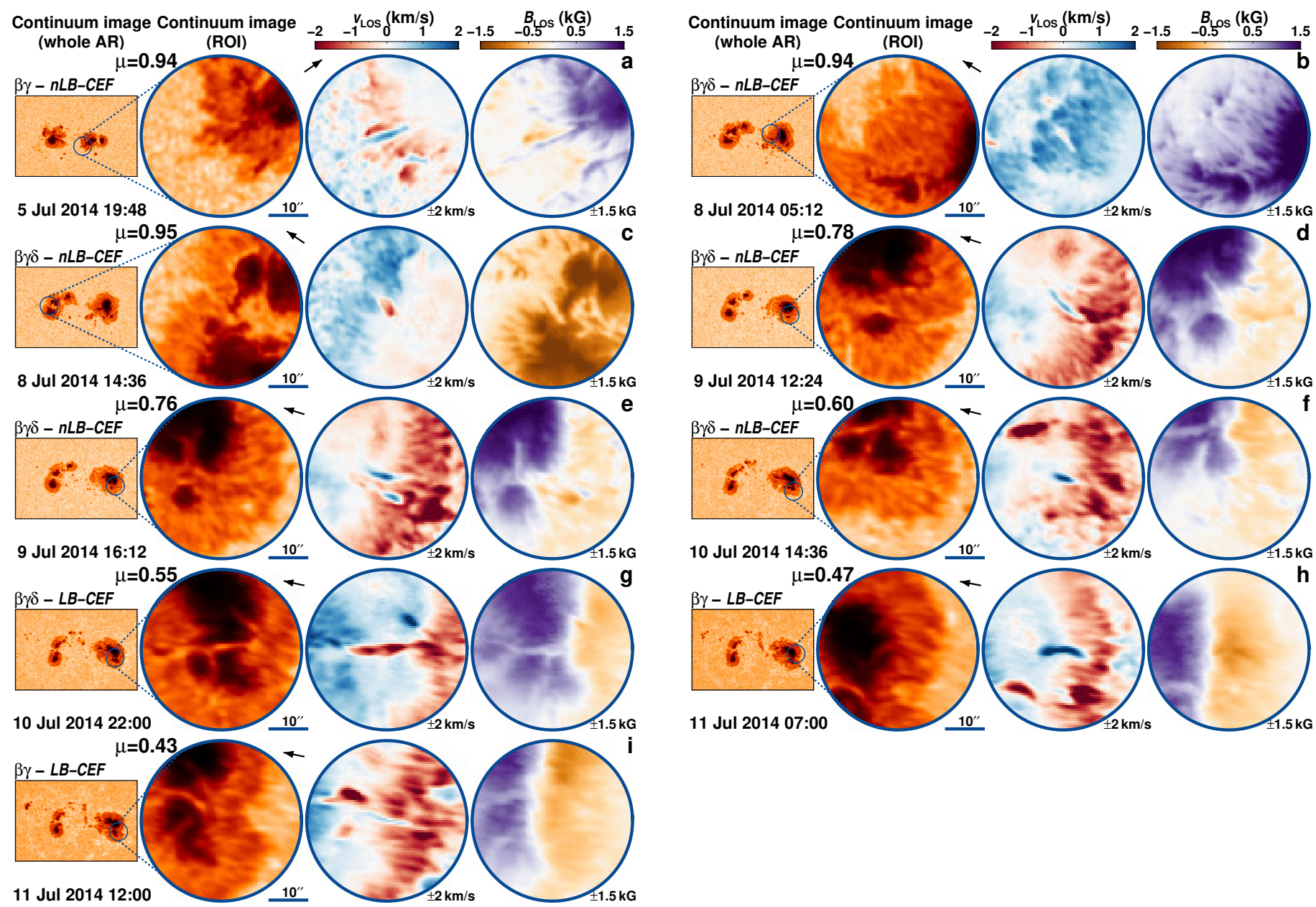

Fig. B.42. AR 12108 followed for 9.9 days from 2-Jul-2014 starting at 06:24 UT. 
J. S. Castellanos Durán et al.: How rare are counter Evershed flows?

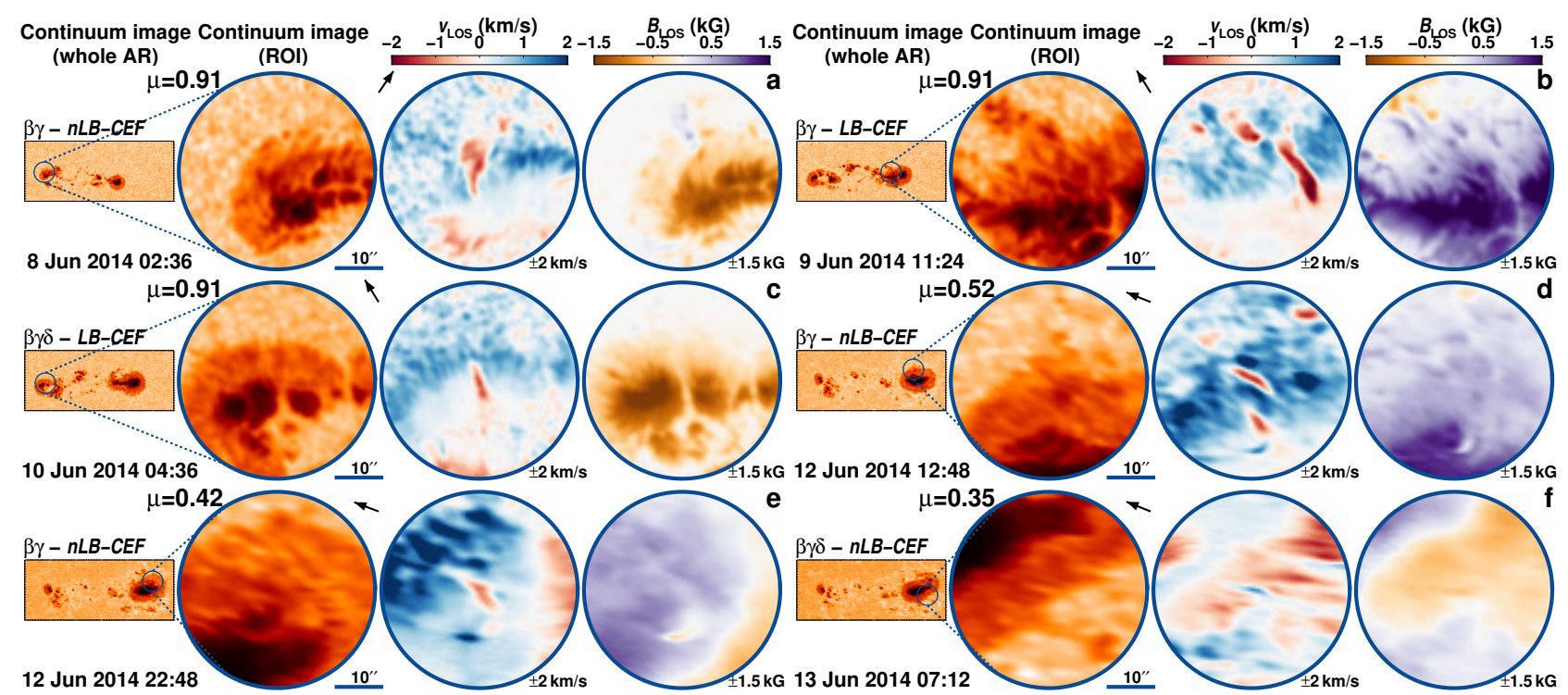

Fig. B.43. AR 12085 followed for 7.1 days from 6-Jun-2014 starting at 11:24 UT.

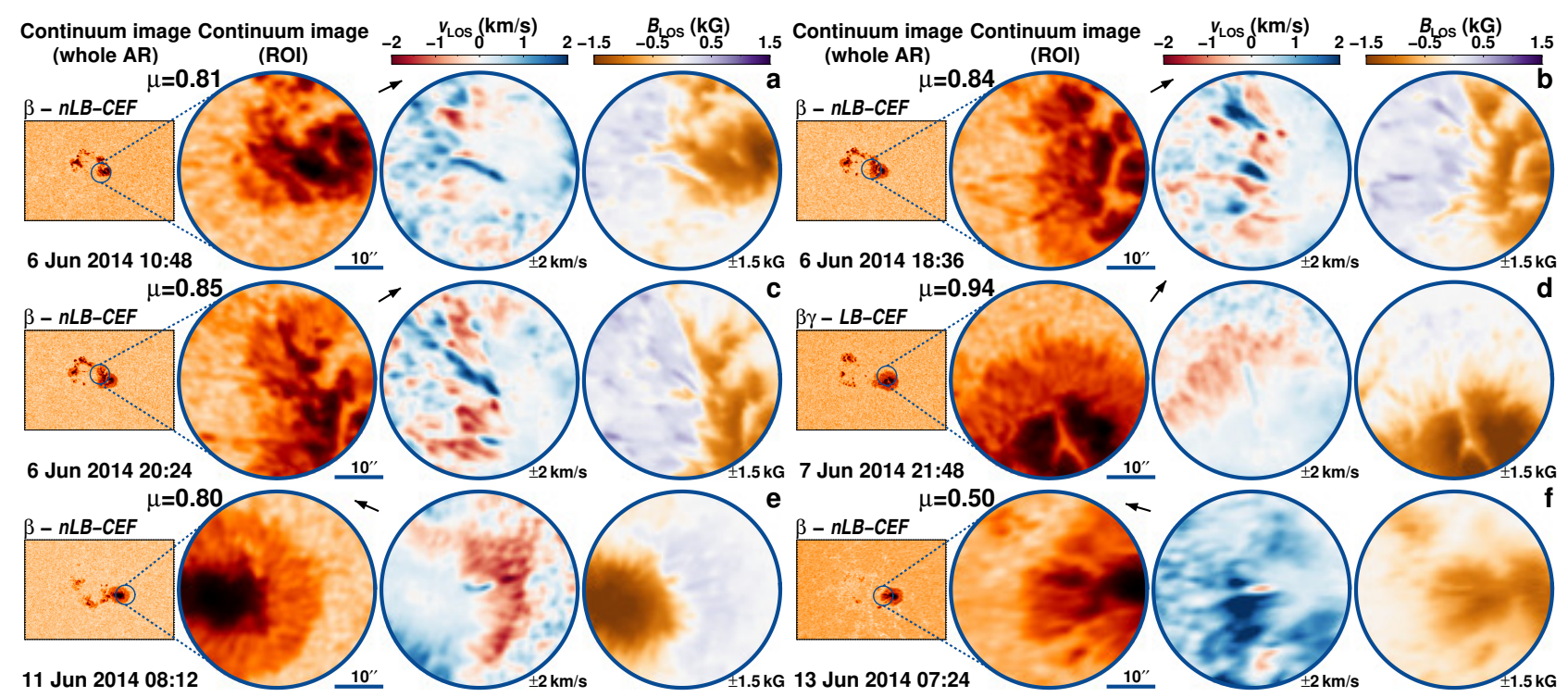

Fig. B.44. AR 12082 followed for 8.2 days from 5-Jun-2014 starting at 08:24 UT.
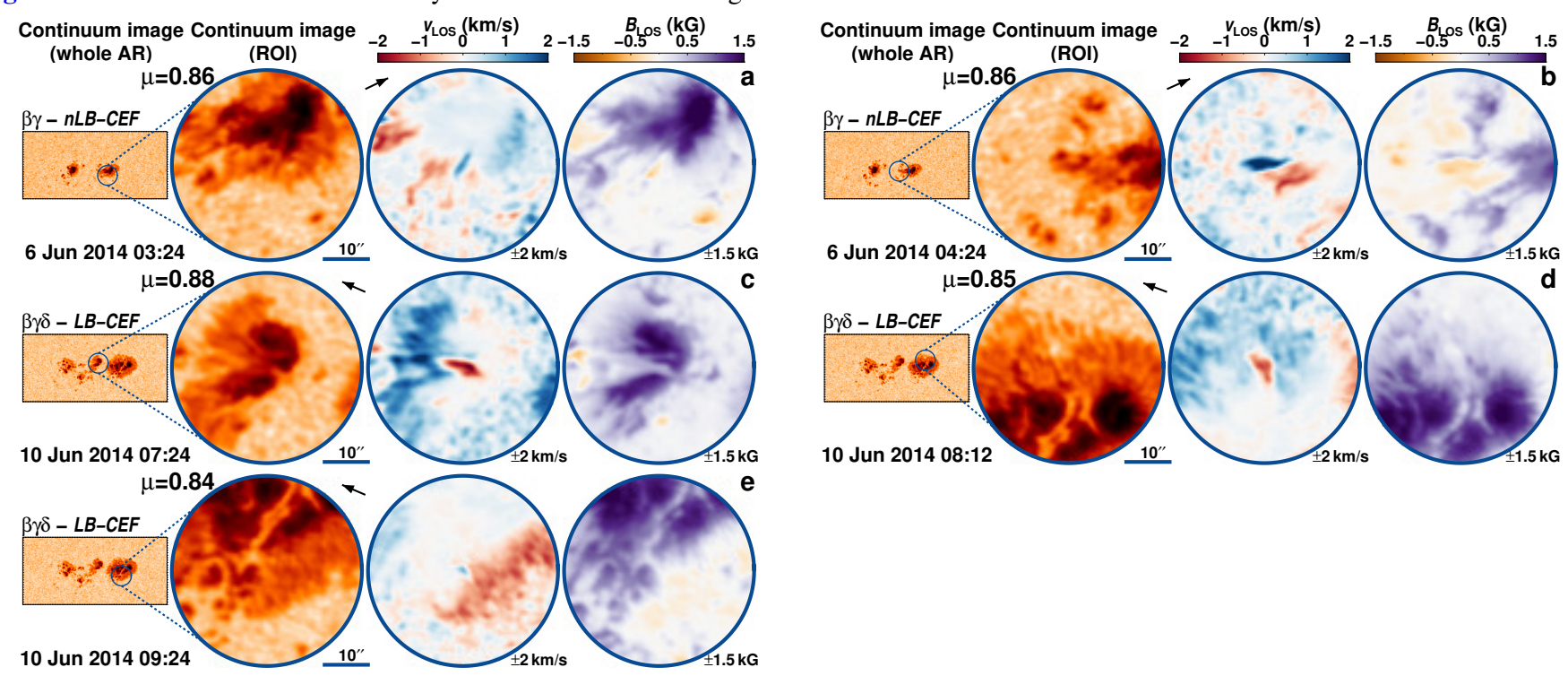

Fig. B.45. AR 12080 followed for 8.8 days from 4-Jun-2014 starting at 19:24 UT. 


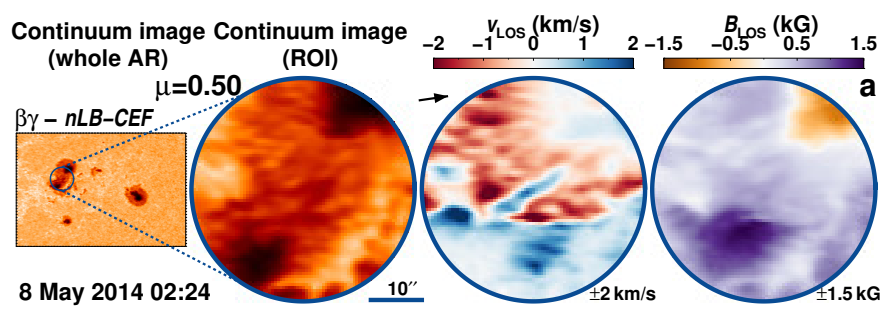

Fig. B.46. AR 12056 followed for 10.5 days from 6-May-2014 starting at 21:48 UT.
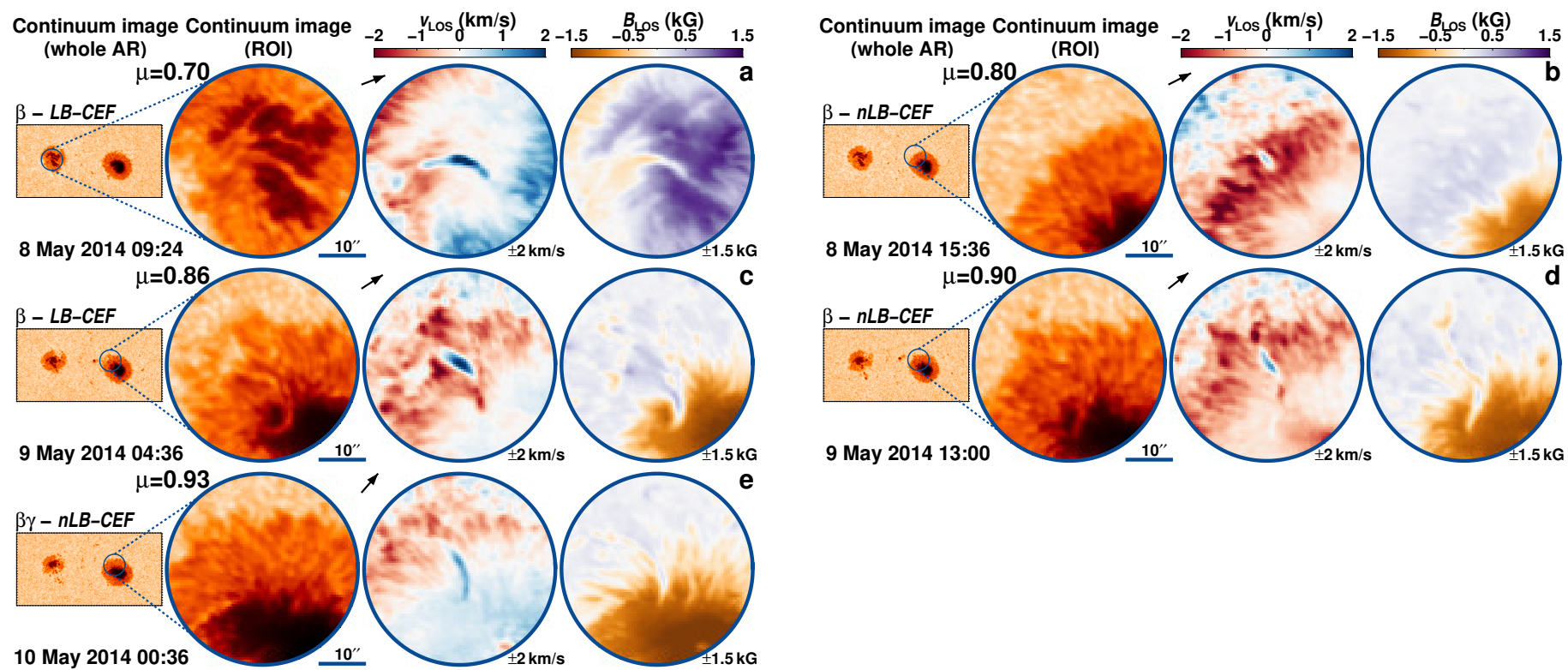

Fig. B.47. AR 12055 followed for 10.2 days from 6-May-2014 starting at 01:48 UT.

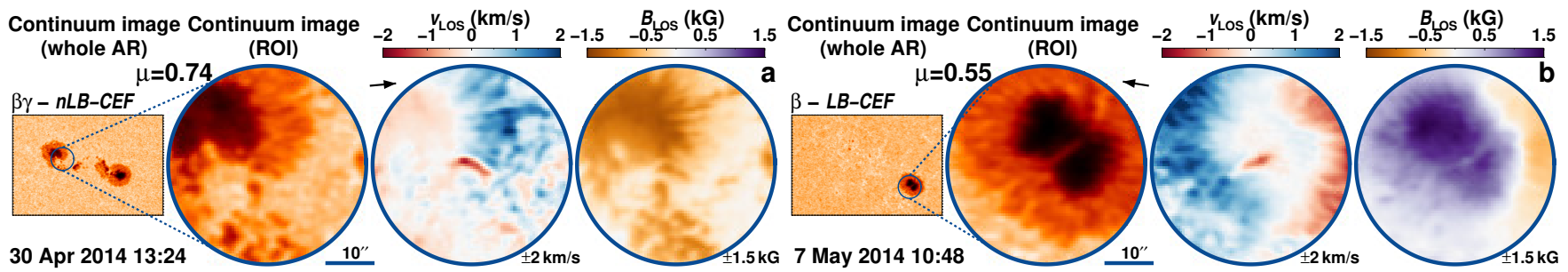

Fig. B.48. AR 12049 followed for 10.9 days from 27-Apr-2014 starting at 22:12 UT.
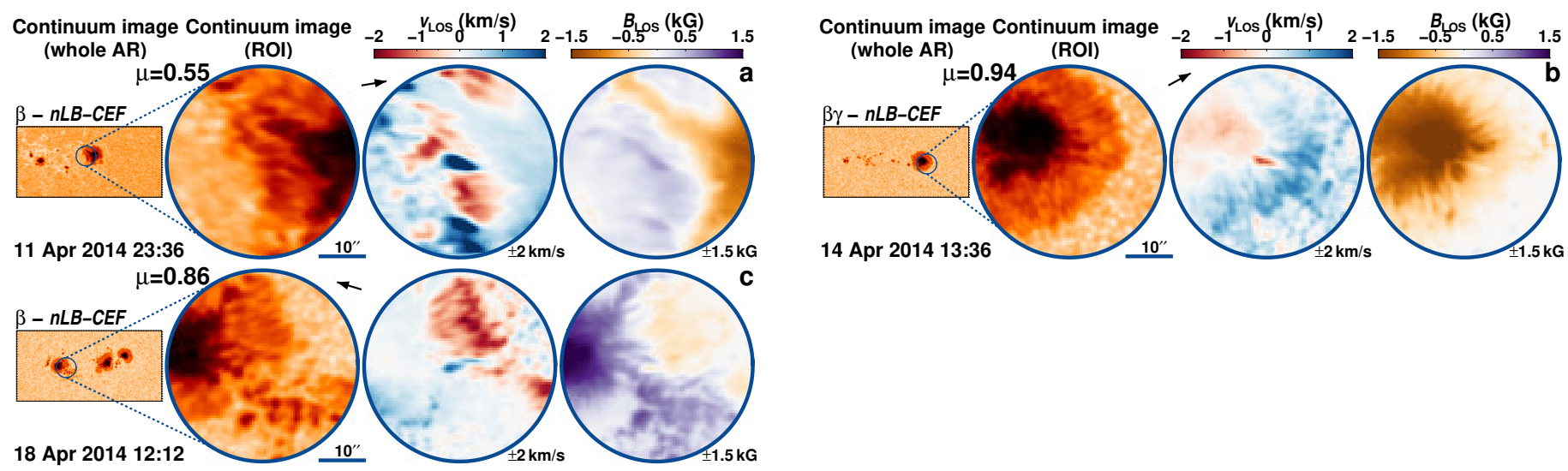

Fig. B.49. AR 12034 followed for 9.6 days from 11-Apr-2014 starting at 09:00 UT. 


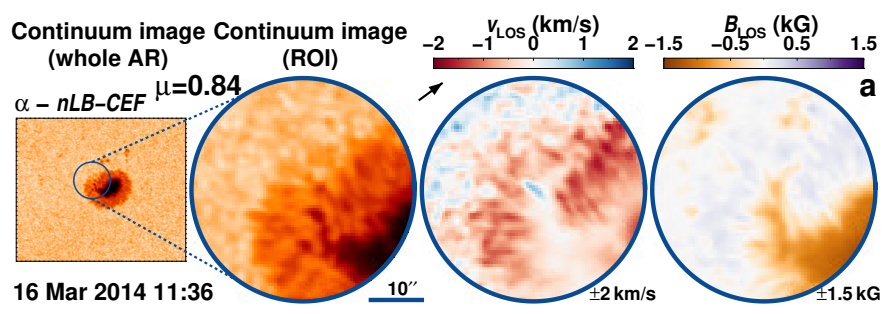

Fig. B.50. AR 12005 followed for 10.5 days from 13-Mar-2014 starting at 02:00 UT.

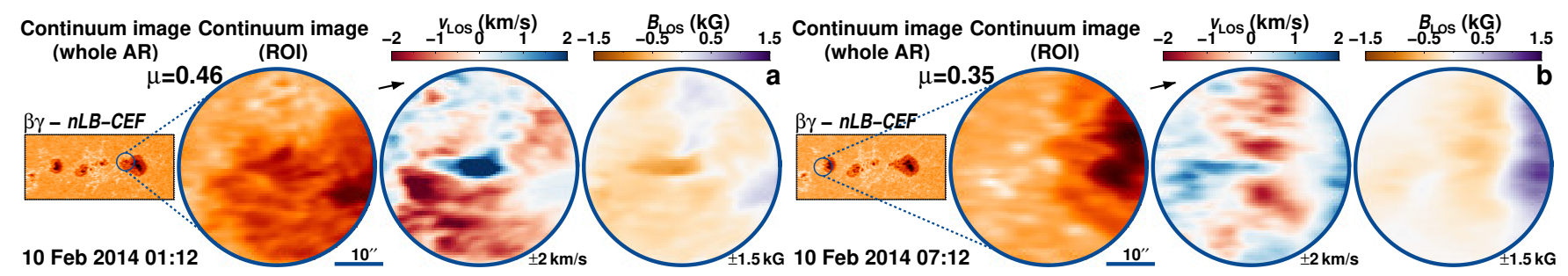

Fig. B.51. AR 11976 followed for 11.4 days from 9-Feb-2014 starting at 03:12 UT.
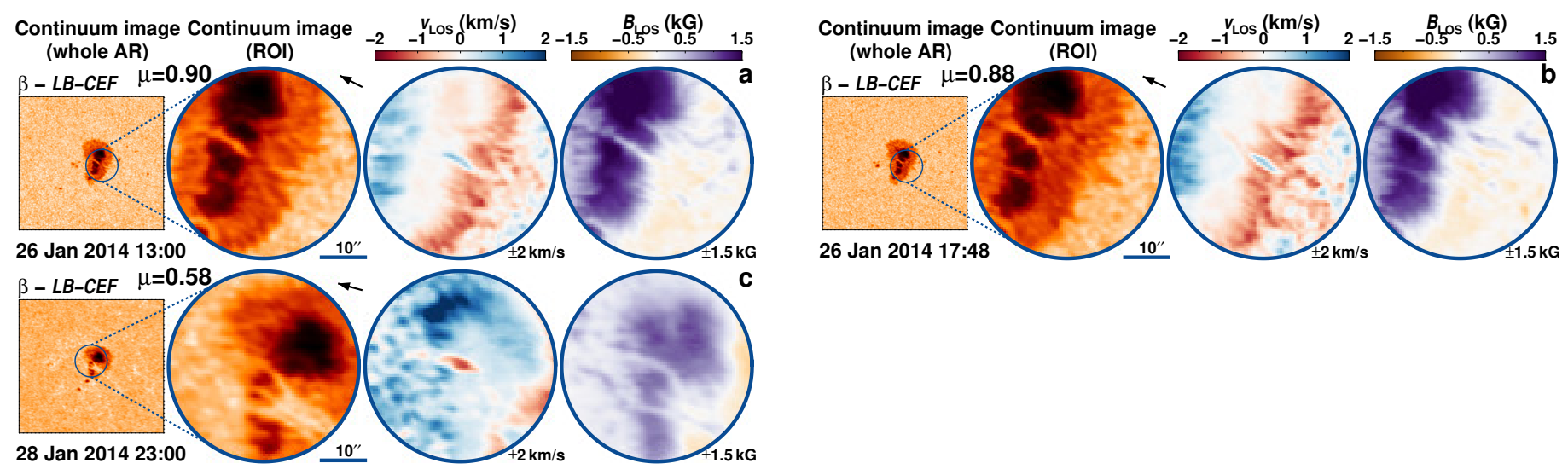

Fig. B.52. AR 11960 followed for 11.4 days from 19-Jan-2014 starting at 03:00 UT. 


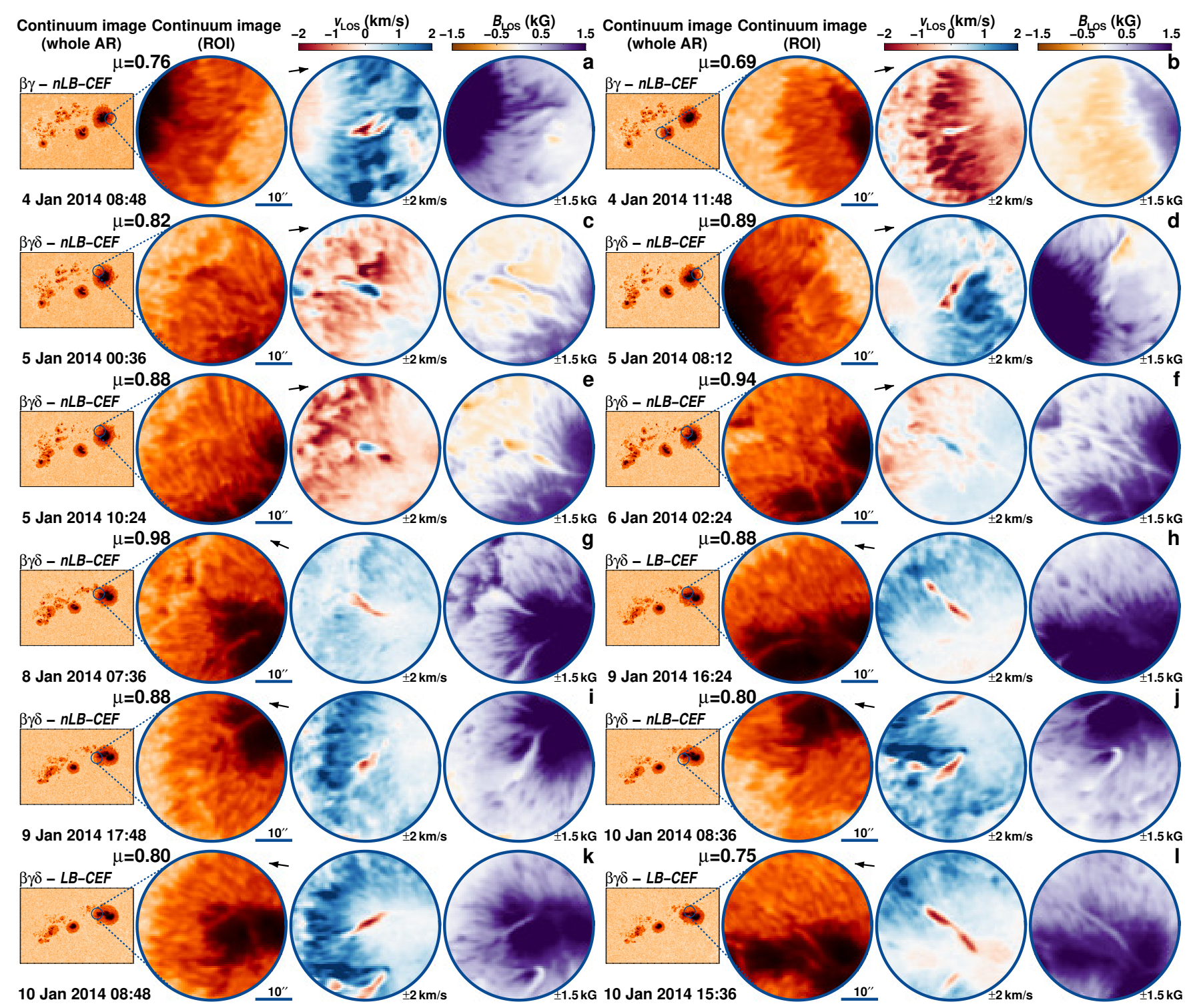

Fig. B.53. AR 11944 followed for 8.3 days from 3-Jan-2014 starting at 22:12 UT.
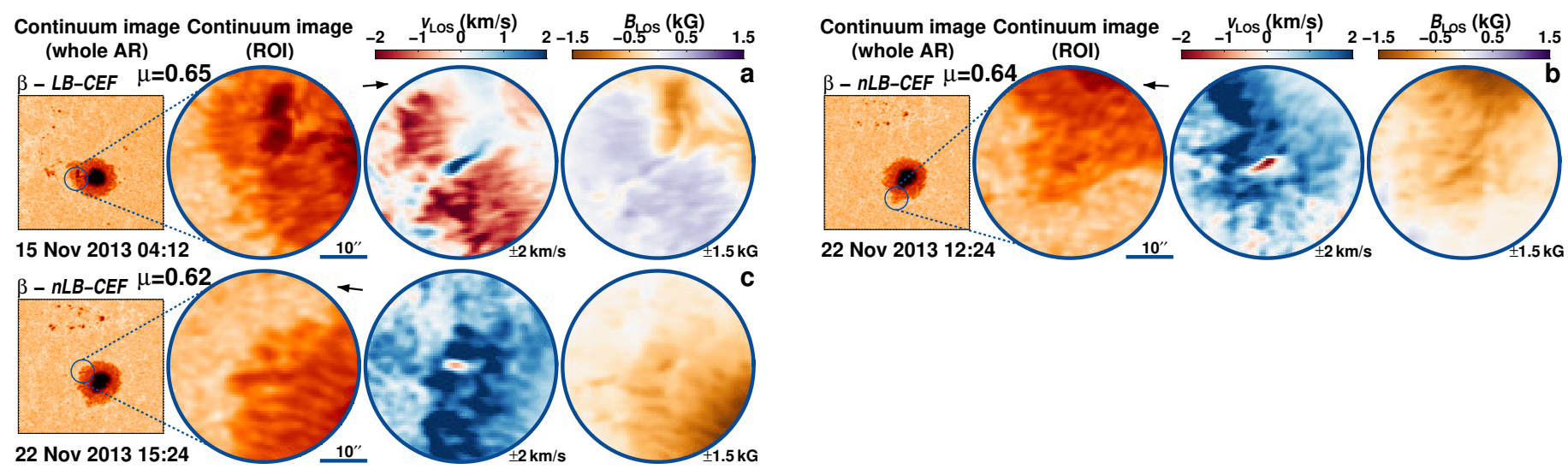

Fig. B.54. AR 11899 followed for 10.8 days from 13-Nov-2013 starting at 07:48 UT. 

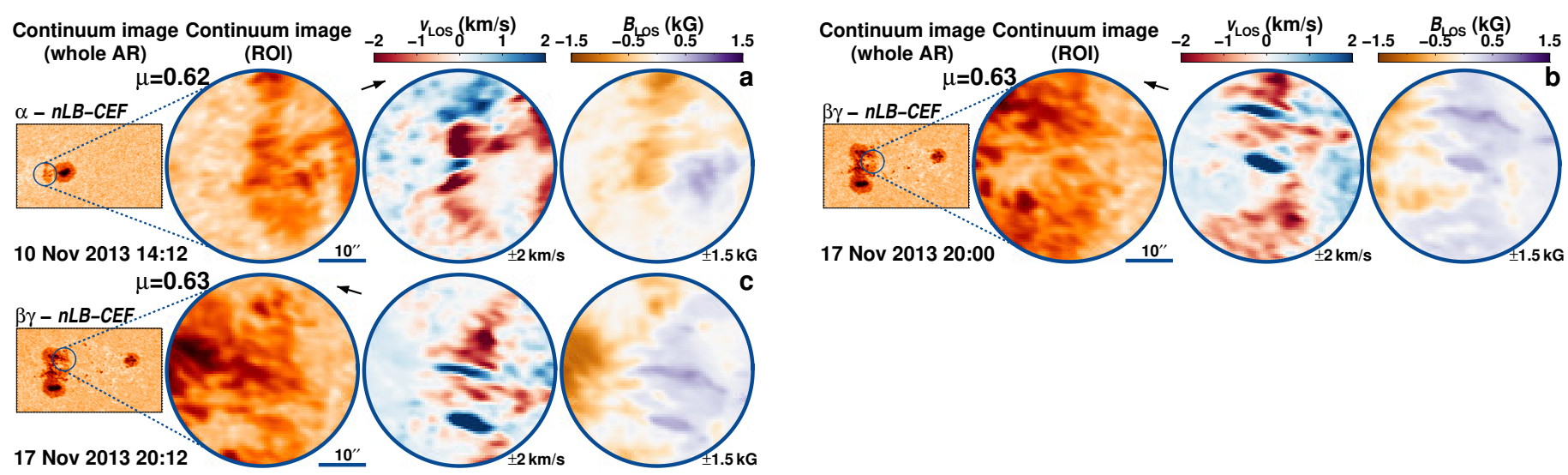

Fig. B.55. AR 11893 followed for 10.5 days from 8-Nov-2013 starting at 12:36 UT.
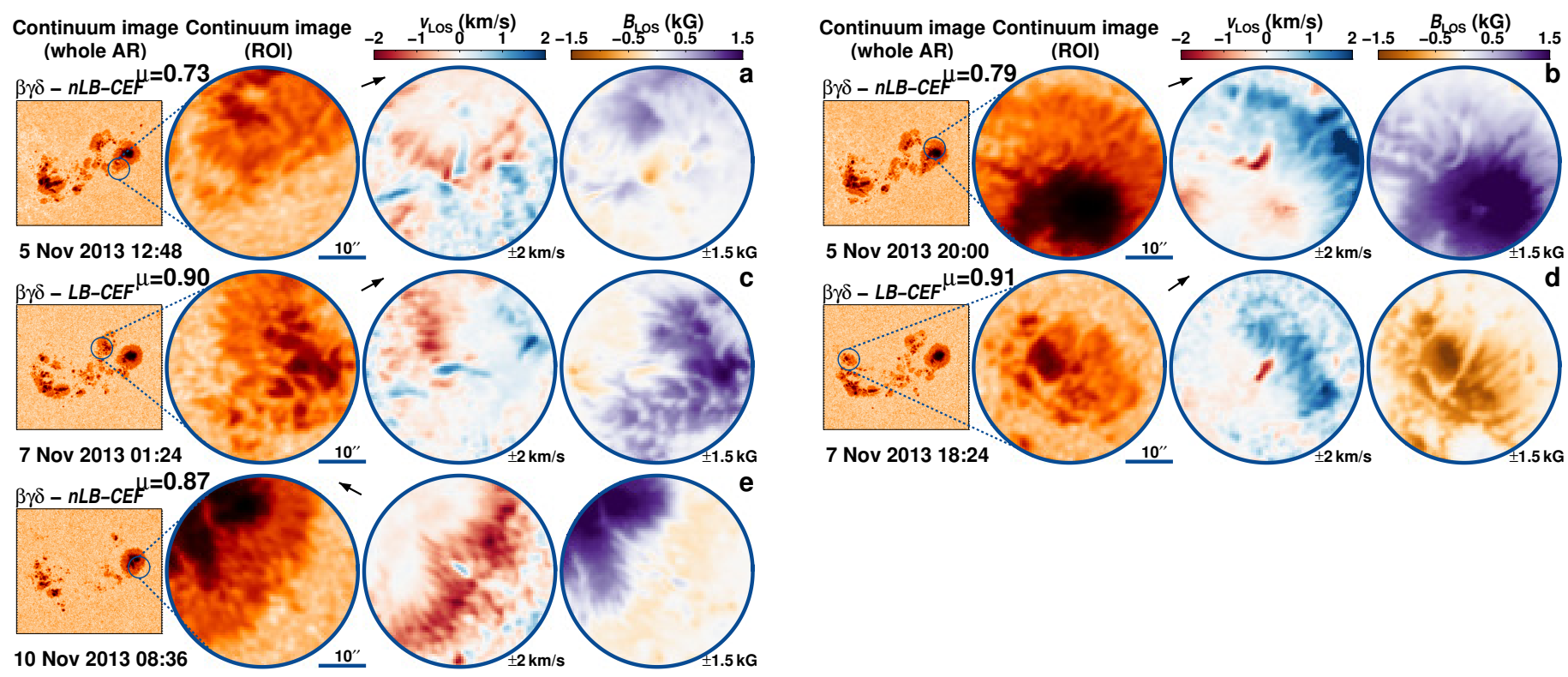

Fig. B.56. AR 11890 followed for 6.5 days from 4-Nov-2013 starting at 12:00 UT.
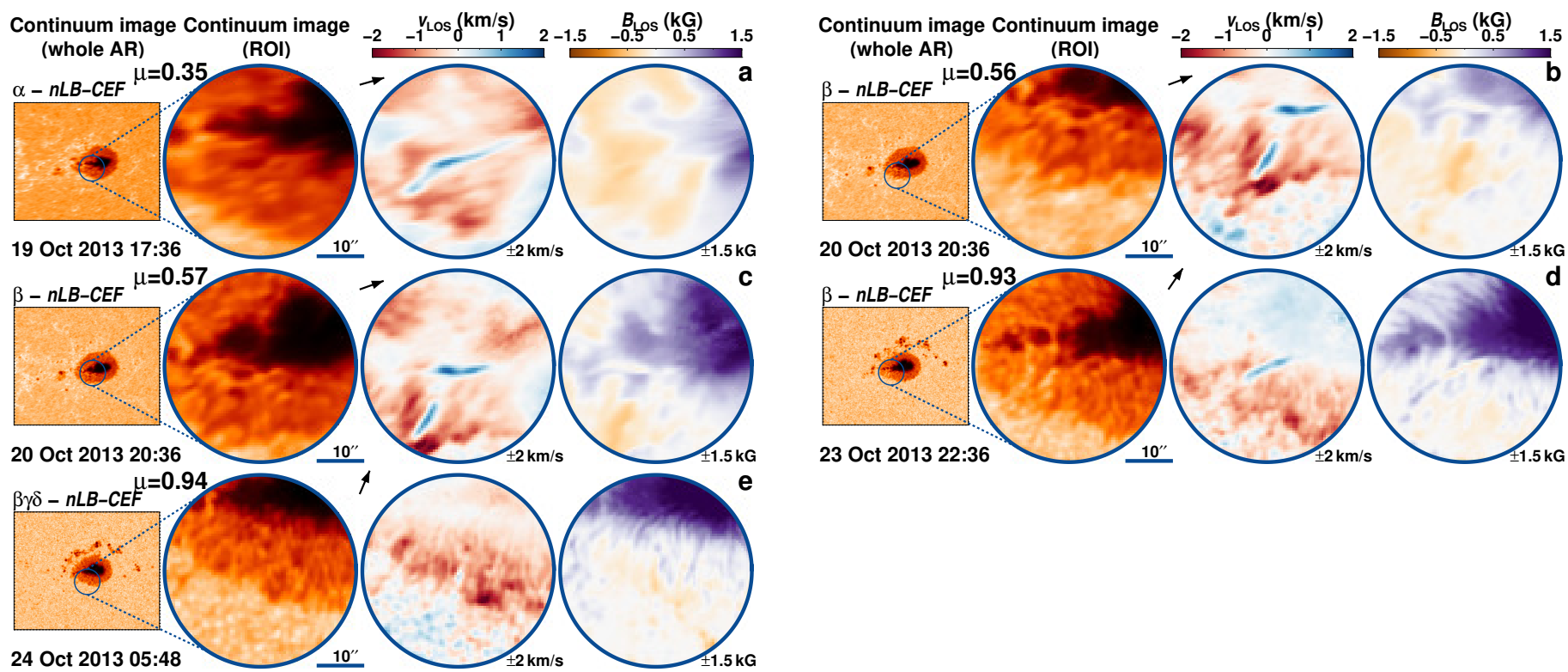

Fig. B.57. AR 11877 followed for 10.5 days from 19-Oct-2013 starting at 15:00 UT. 


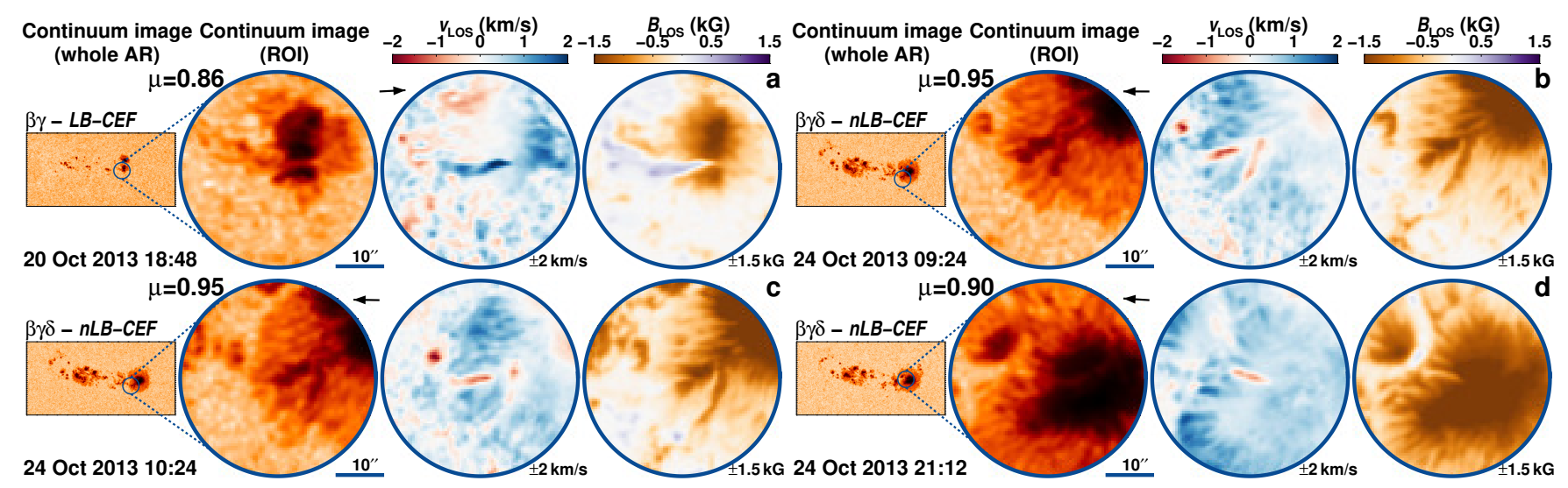

Fig. B.58. AR 11875 followed for 10.7 days from 18-Oct-2013 starting at 00:00 UT.
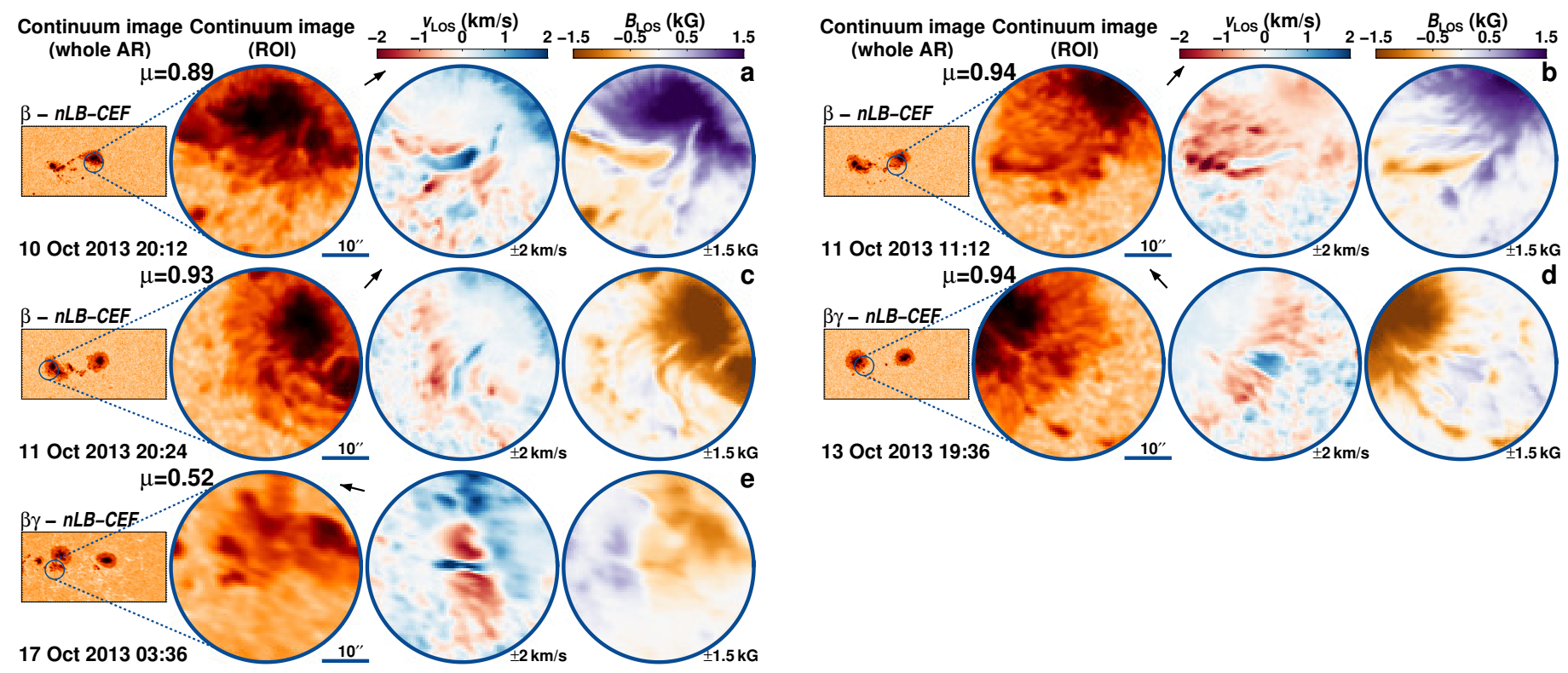

Fig. B.59. AR 11861 followed for 8.1 days from 9-Oct-2013 starting at 22:48 UT.
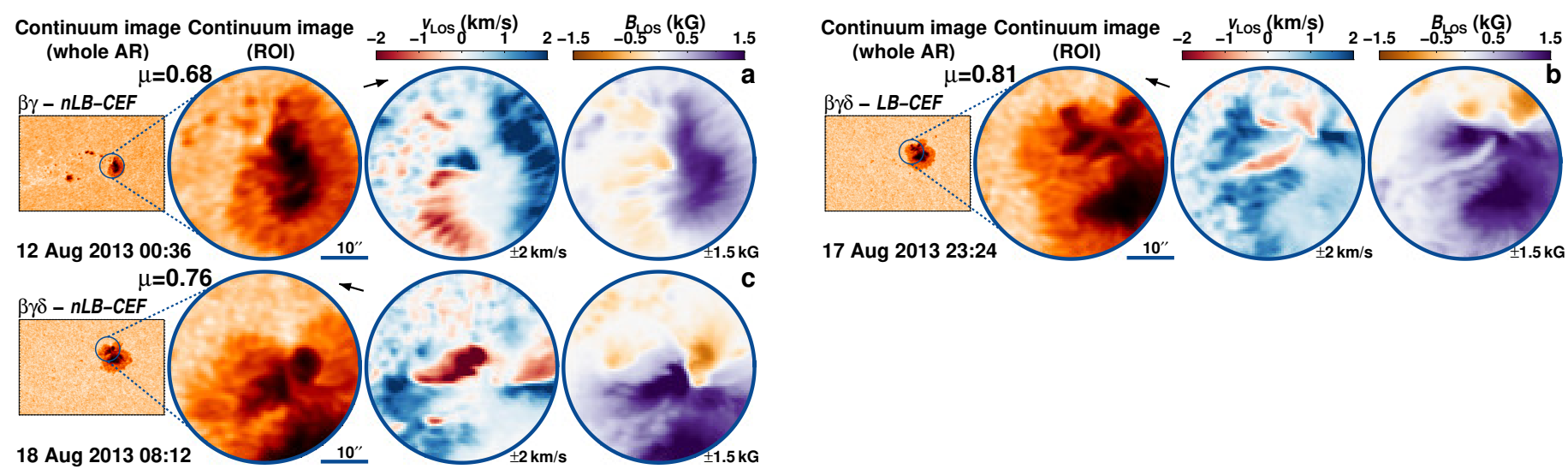

Fig. B.60. AR 11818 followed for 9.2 days from 11-Aug-2013 starting at 10:00 UT. 


\section{J. S. Castellanos Durán et al.: How rare are counter Evershed flows?}

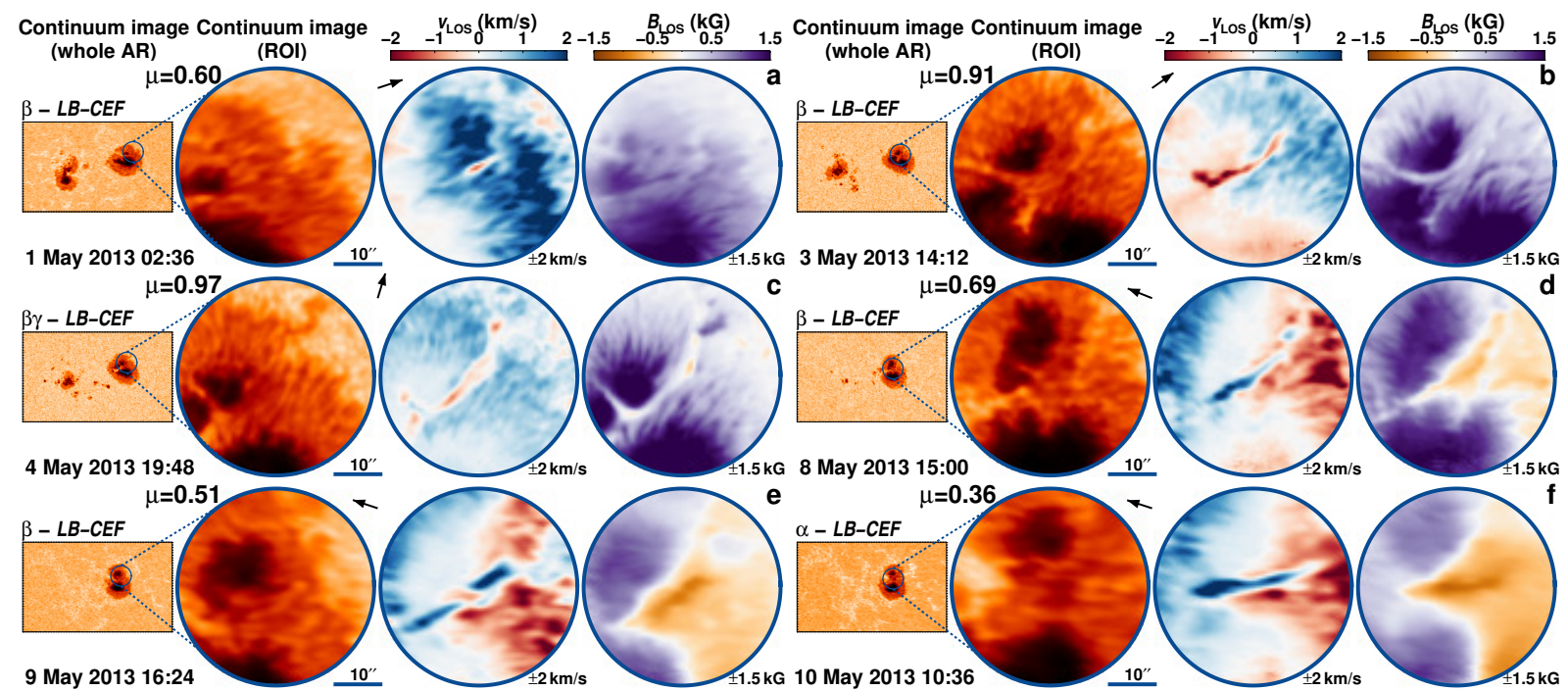

Fig. B.61. AR 11734 followed for 10.0 days from 1-May-2013 starting at 01:00 UT.
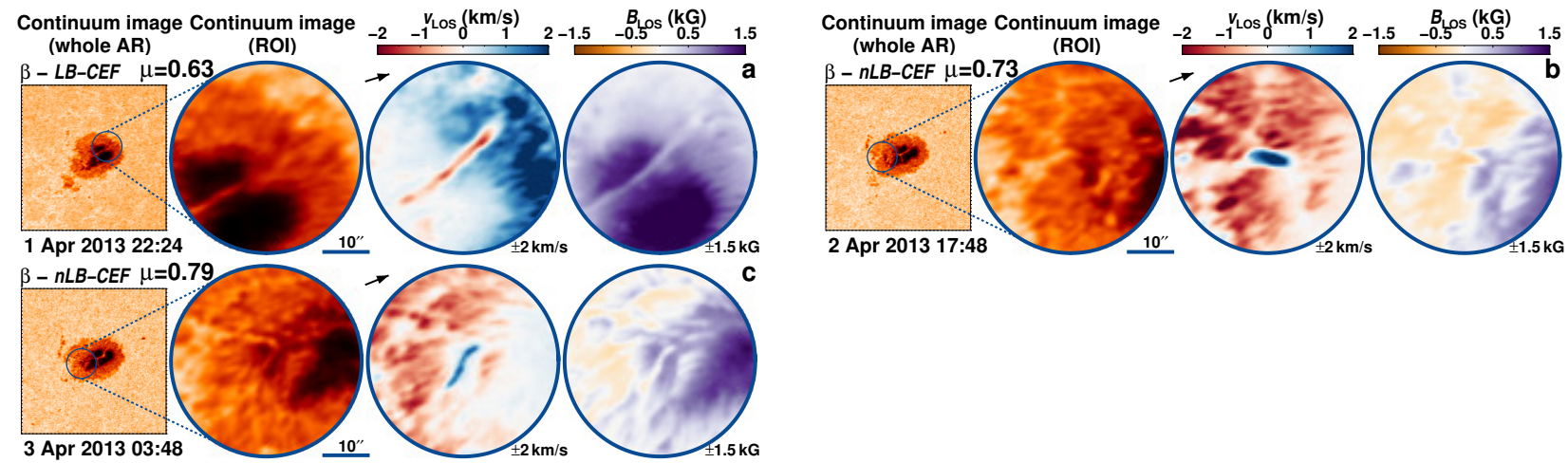

Fig. B.62. AR 11711 followed for 10.7 days from 31-Mar-2013 starting at 01:48 UT.
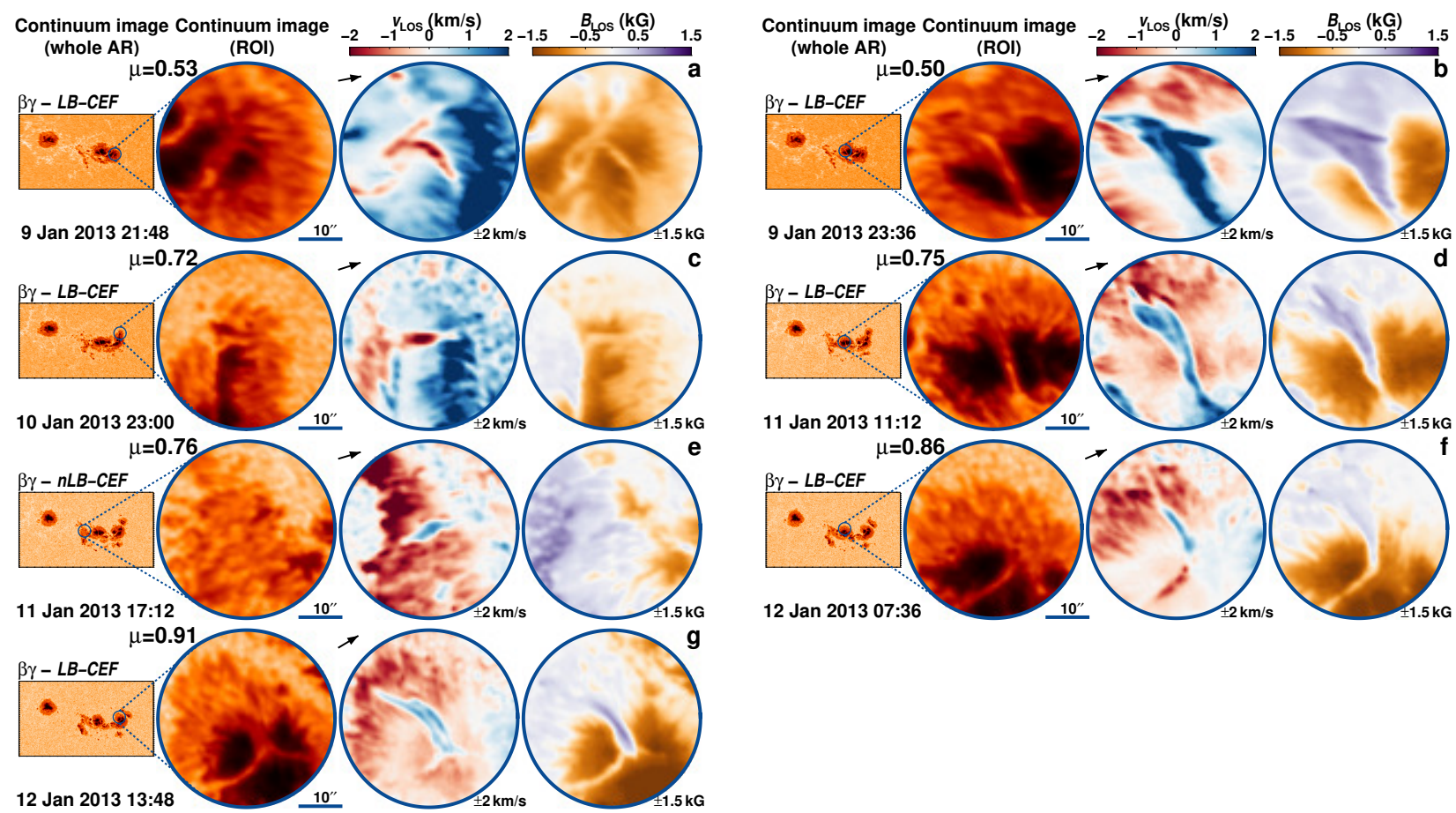

Fig. B.63. AR 11654 followed for 10.0 days from 8-Jan-2013 starting at 15:00 UT. 


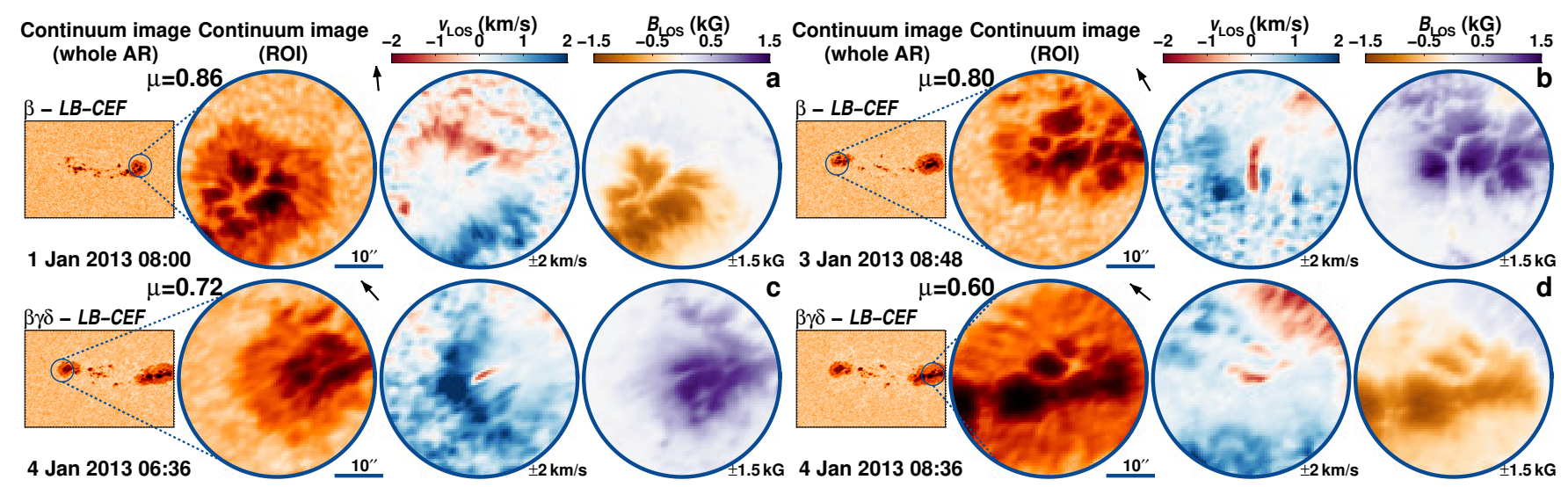

Fig. B.64. AR 11640 followed for 7.7 days from 30-Dec-2012 starting at 02:00 UT.

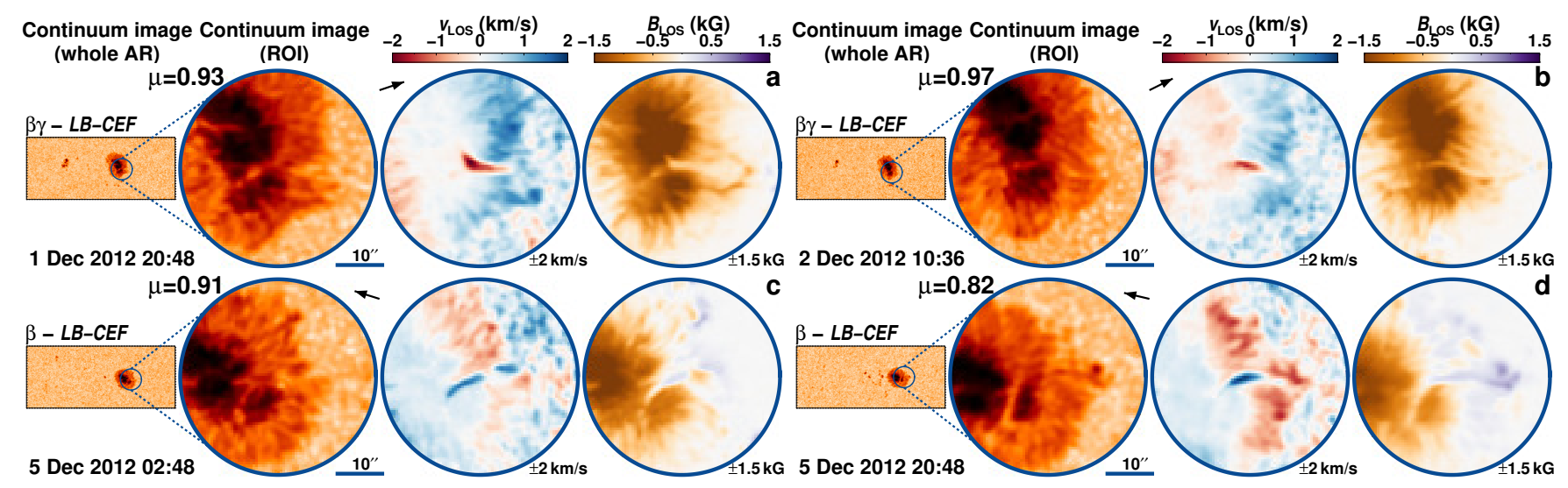

Fig. B.65. AR 11623 followed for 11.1 days from 28-Nov-2012 starting at 00:12 UT.
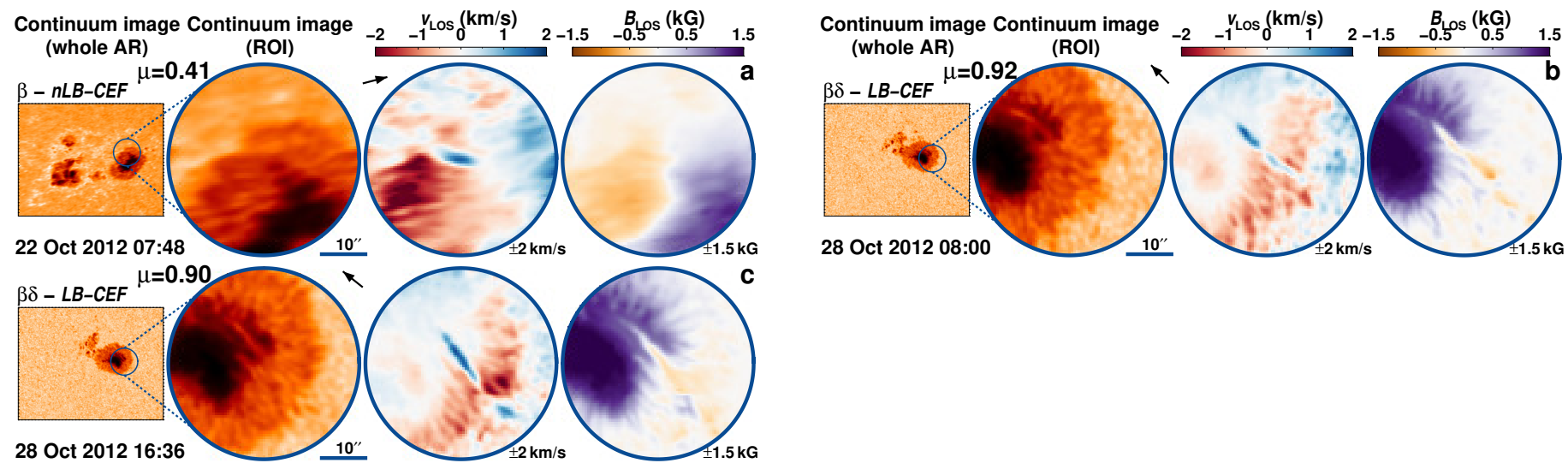

Fig. B.66. AR 11598 followed for 11.1 days from 22-Oct-2012 starting at 00:00 UT.

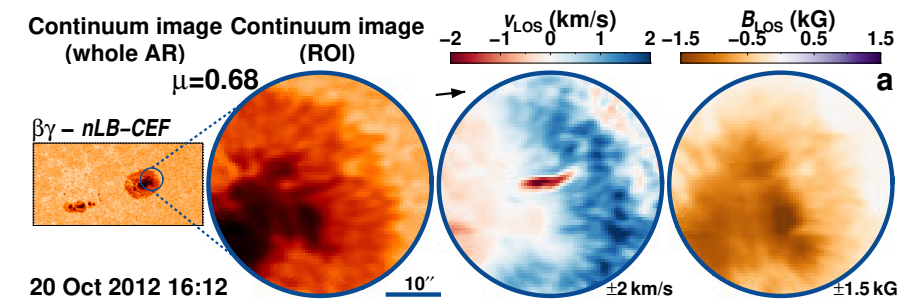

Fig. B.67. AR 11596 followed for 11.1 days from 18-Oct-2012 starting at 18:48 UT. 
J. S. Castellanos Durán et al.: How rare are counter Evershed flows?

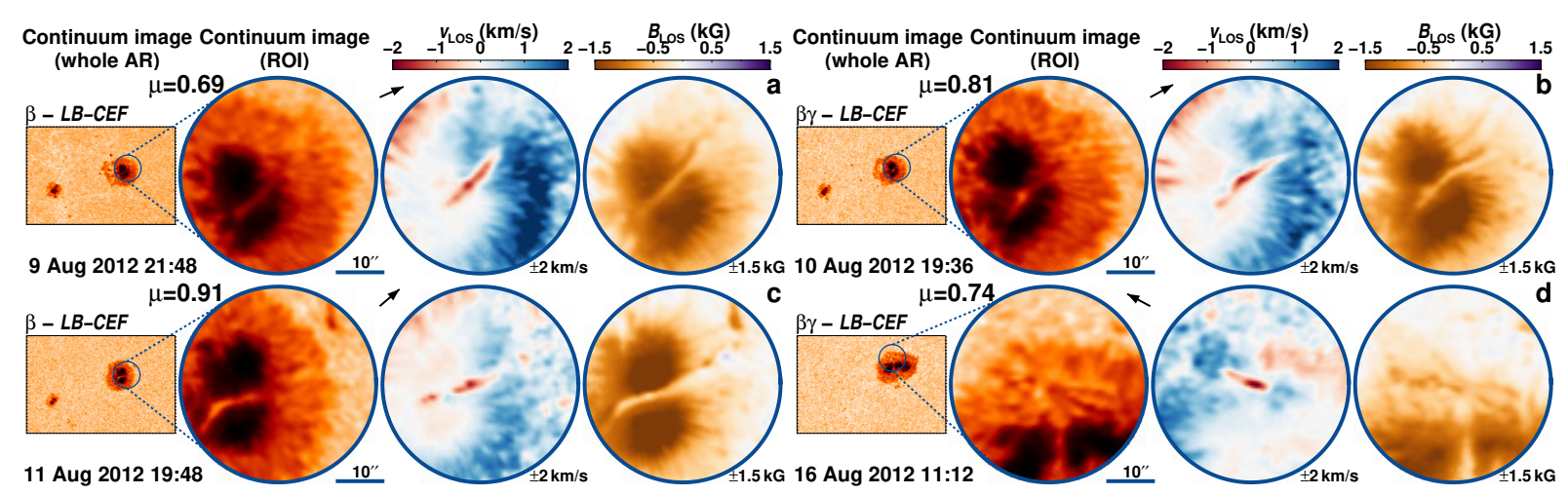

Fig. B.68. AR 11543 followed for 11.0 days from 8-Aug-2012 starting at 00:24 UT.
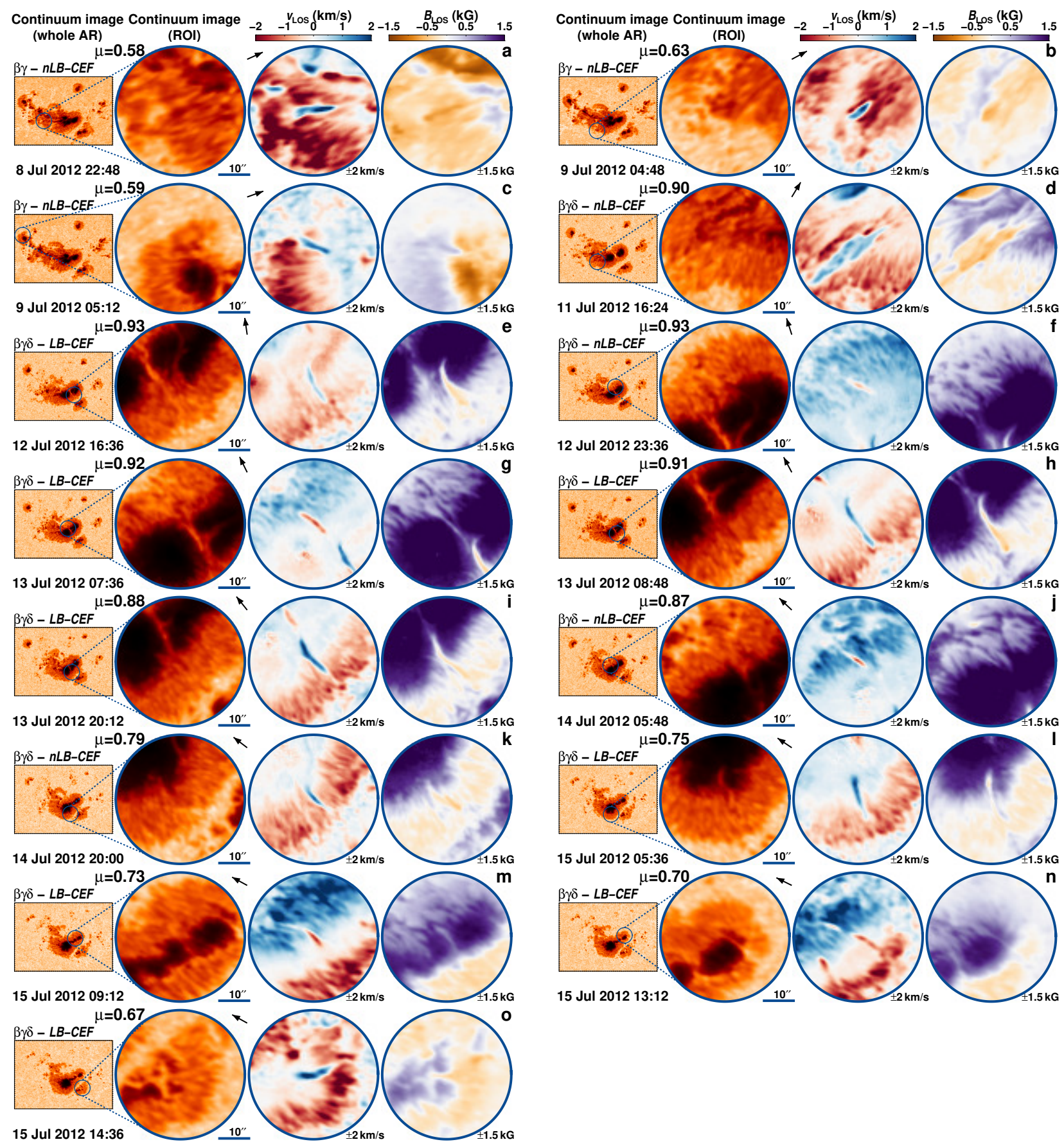

Fig. B.69. AR 11520 followed for 8.0 days from 8-Jul-2012 starting at 18:12 UT. 

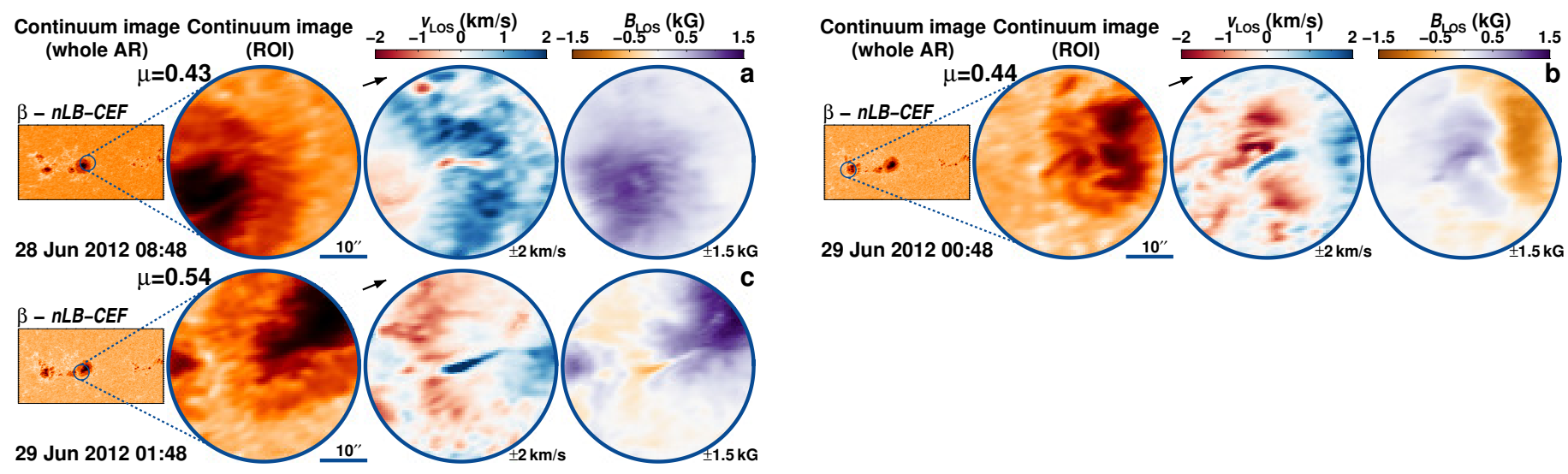

Fig. B.70. AR 11515 followed for 10.5 days from 27-Jun-2012 starting at 20:36 UT.

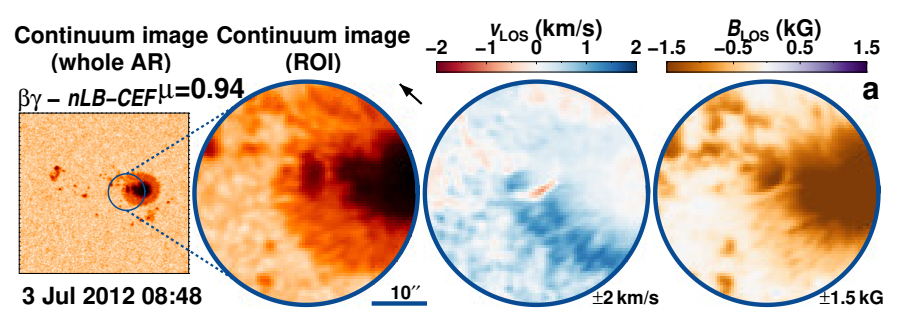

Fig. B.71. AR 11513 followed for 11.3 days from 26-Jun-2012 starting at 17:12 UT.
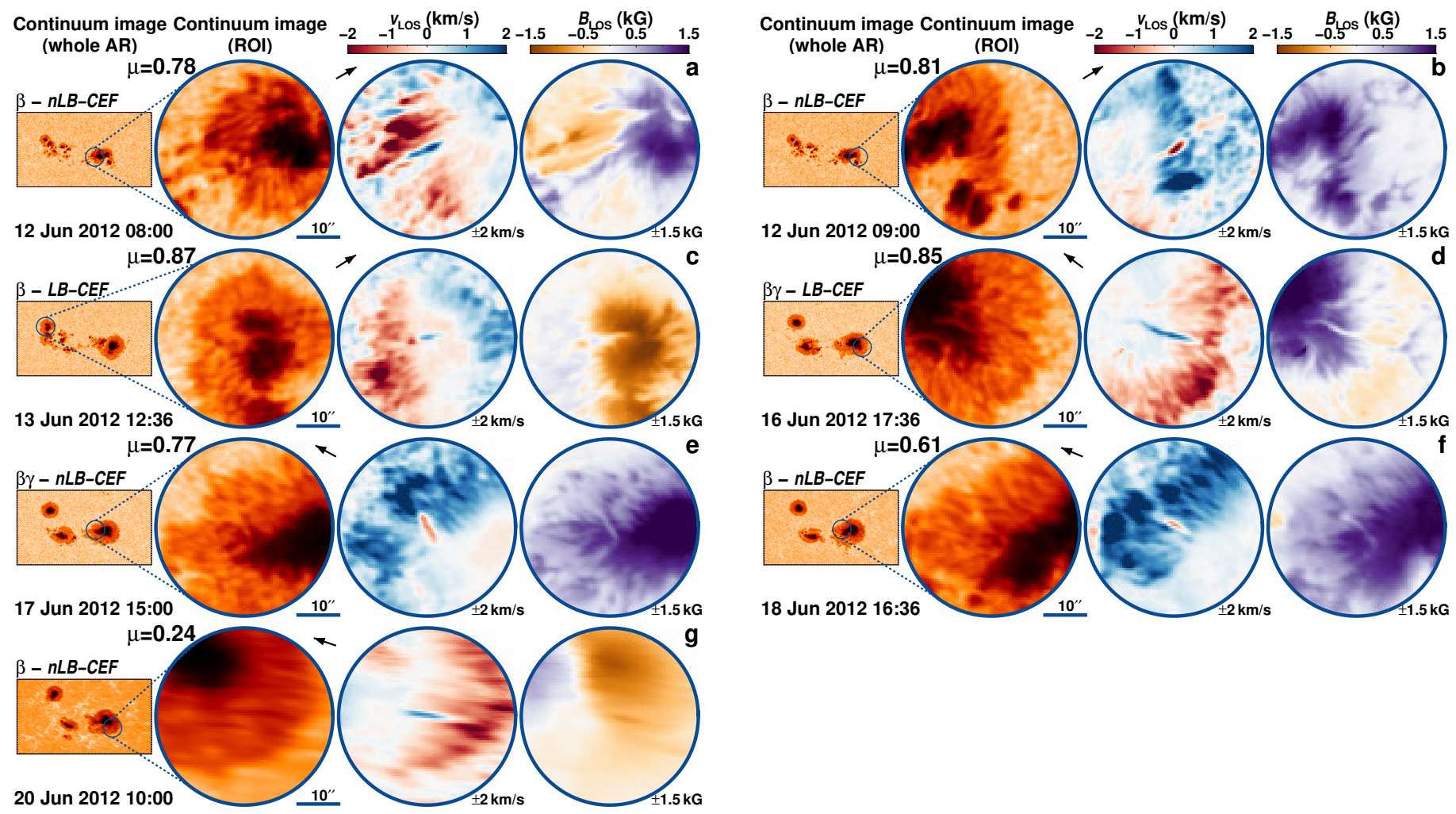

Fig. B.72. AR 11504 followed for 11.3 days from 9-Jun-2012 starting at 10:24 UT. 
J. S. Castellanos Durán et al.: How rare are counter Evershed flows?
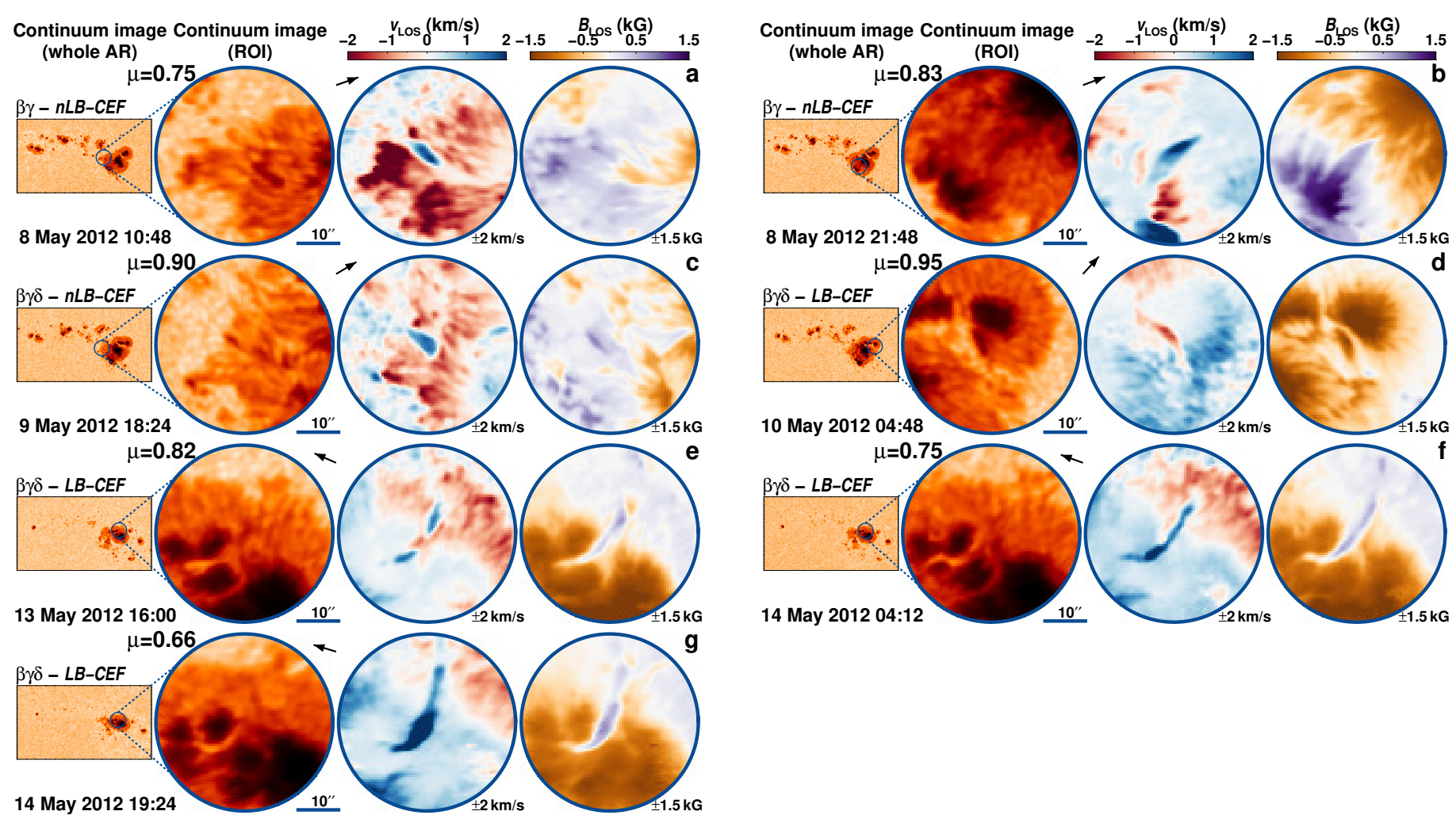

Fig. B.73. AR 11476 followed for 8.0 days from 8-May-2012 starting at 00:00 UT.
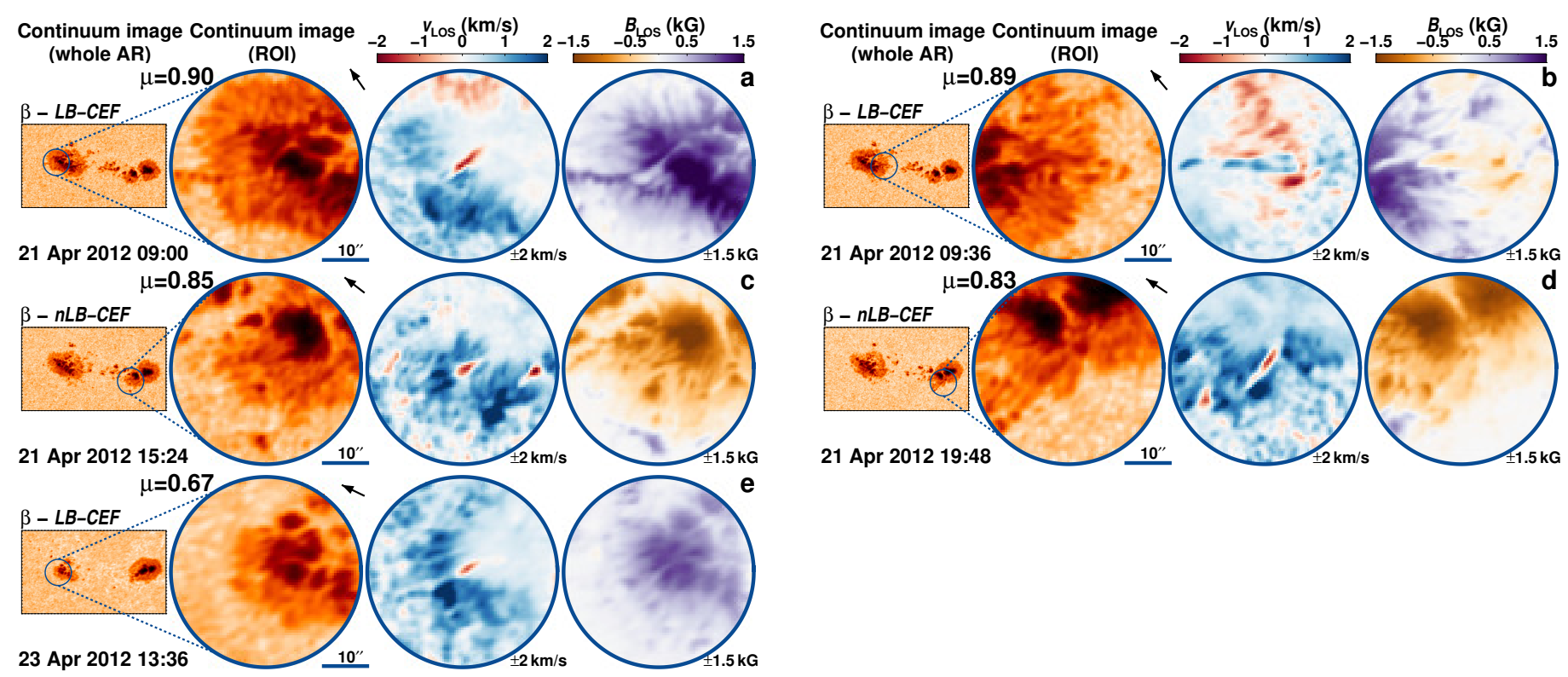

Fig. B.74. AR 11460 followed for 8.5 days from 16-Apr-2012 starting at 19:24 UT. 

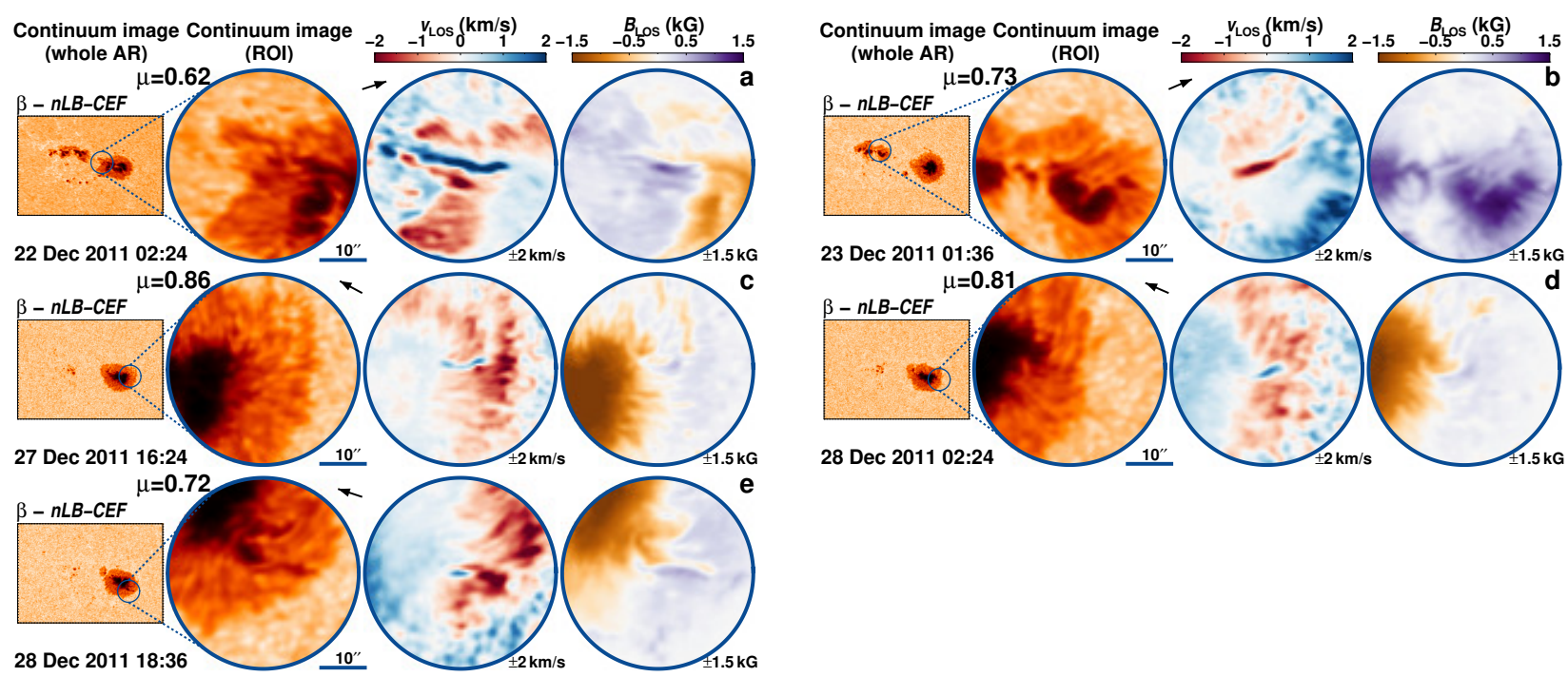

Fig. B.75. AR 11384 followed for 11.4 days from 20-Dec-2011 starting at 03:36 UT.
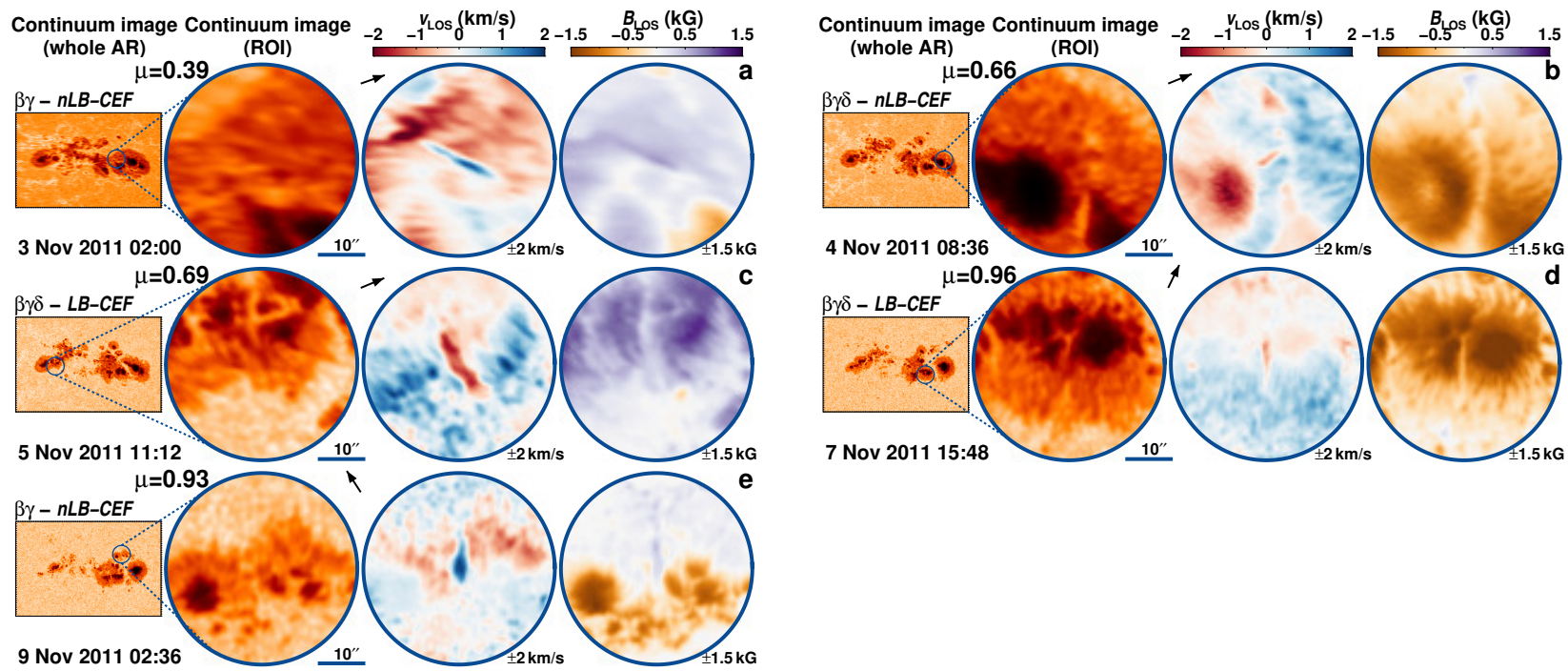

Fig. B.76. AR 11339 followed for 11.6 days from 2-Nov-2011 starting at 10:00 UT.

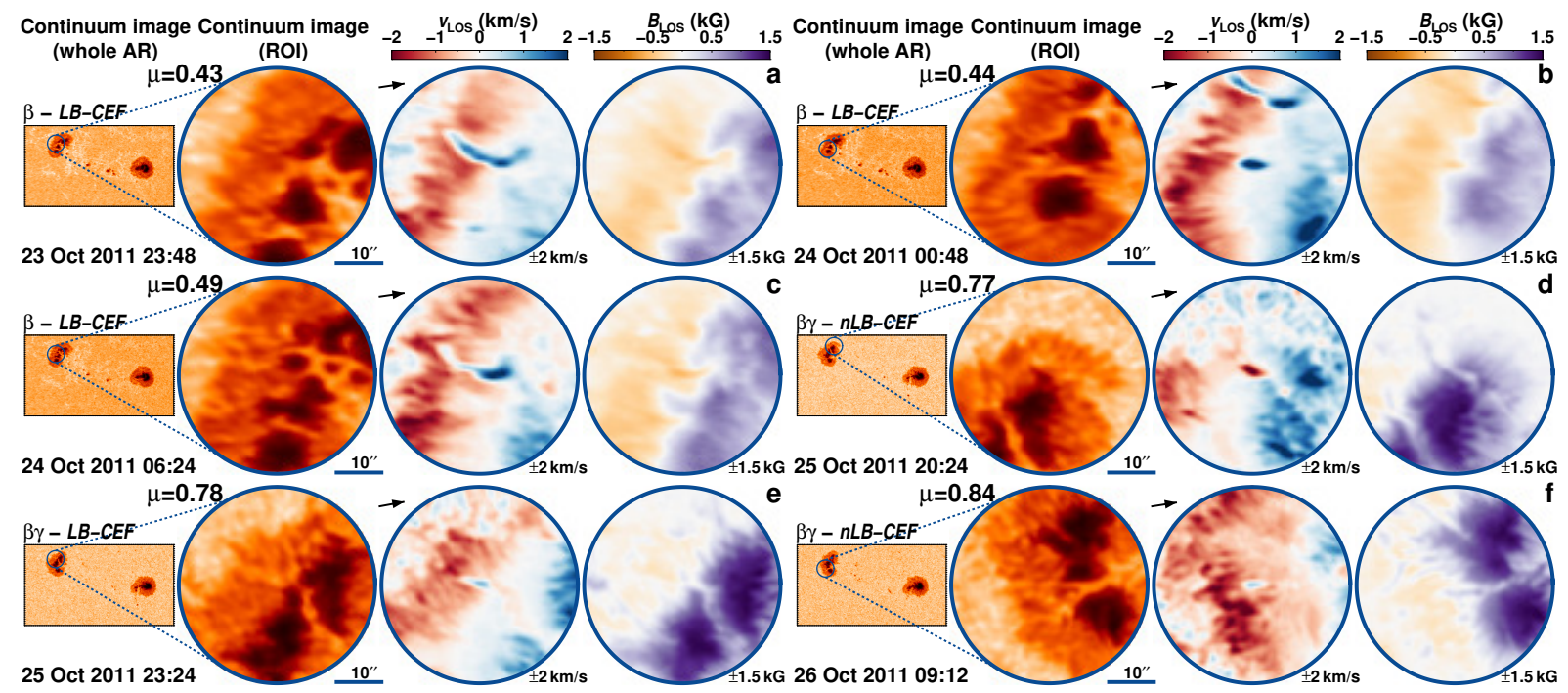

Fig. B.77. AR 11330 followed for 10.1 days from 22-Oct-2011 starting at 16:36 UT. 


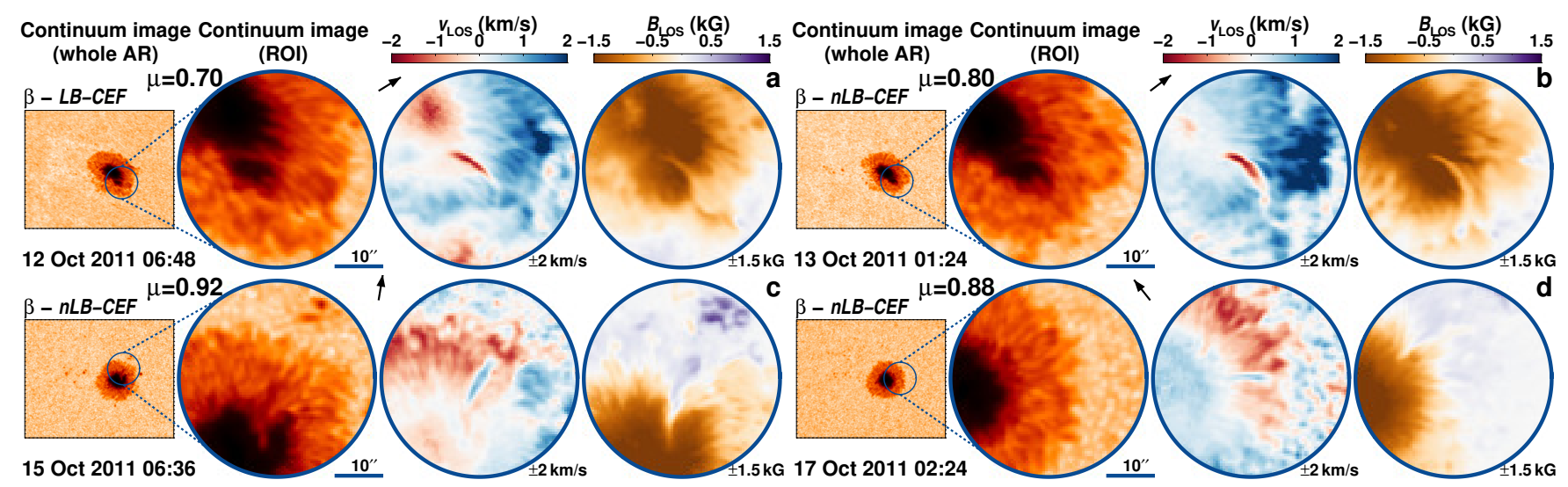

Fig. B.78. AR 11314 followed for 8.7 days from 11-Oct-2011 starting at 11:36 UT.
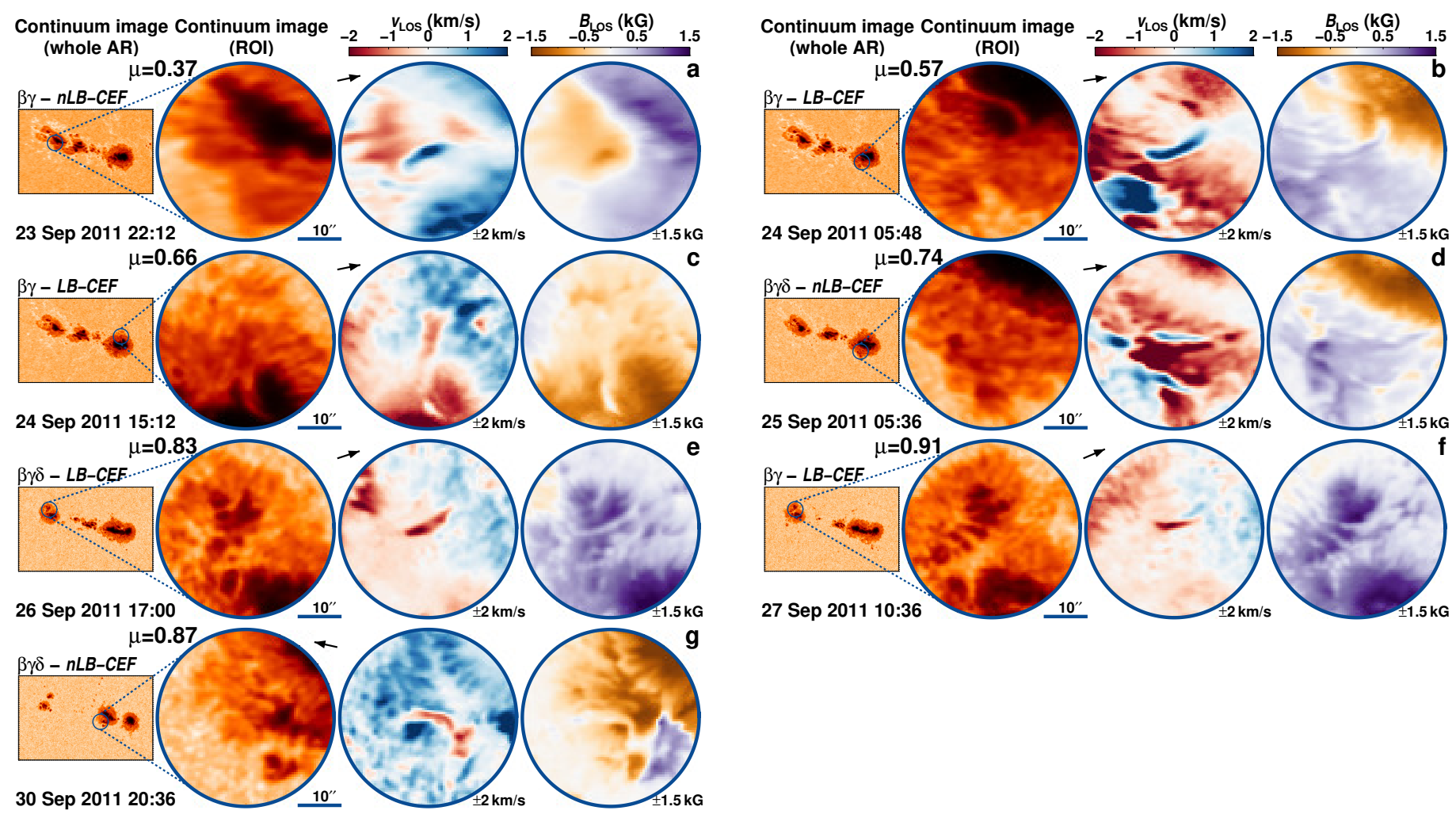

Fig. B.79. AR 11302 followed for 11.1 days from 23-Sep-2011 starting at 05:24 UT. 

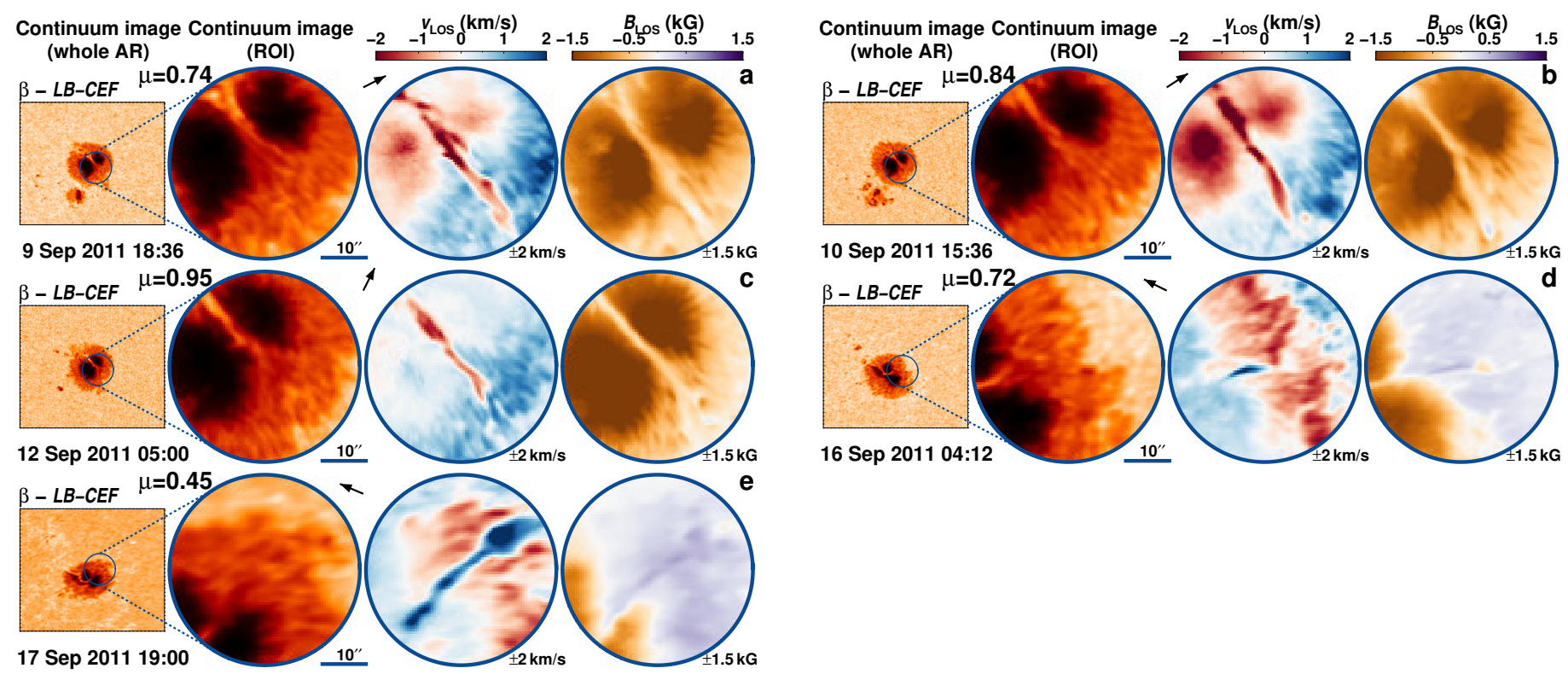

Fig. B.80. AR 11289 followed for 10.3 days from 8-Sep-2011 starting at 06:12 UT.

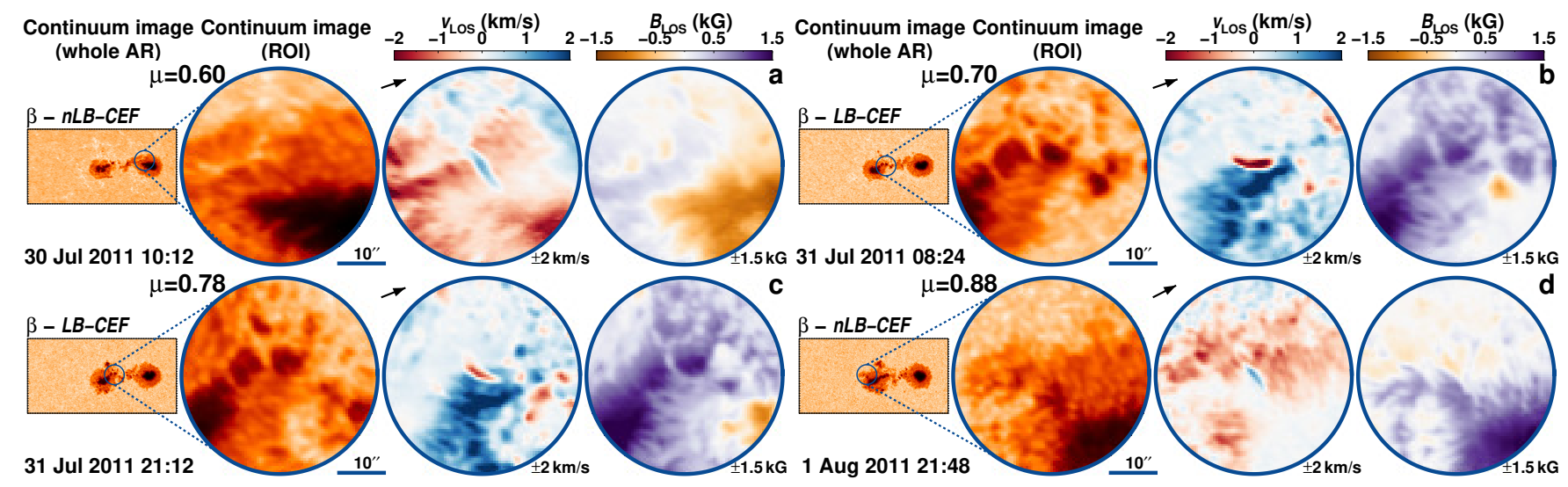

Fig. B.81. AR 11263 followed for 9.5 days from 30-Jul-2011 starting at 00:12 UT. 
J. S. Castellanos Durán et al.: How rare are counter Evershed flows?

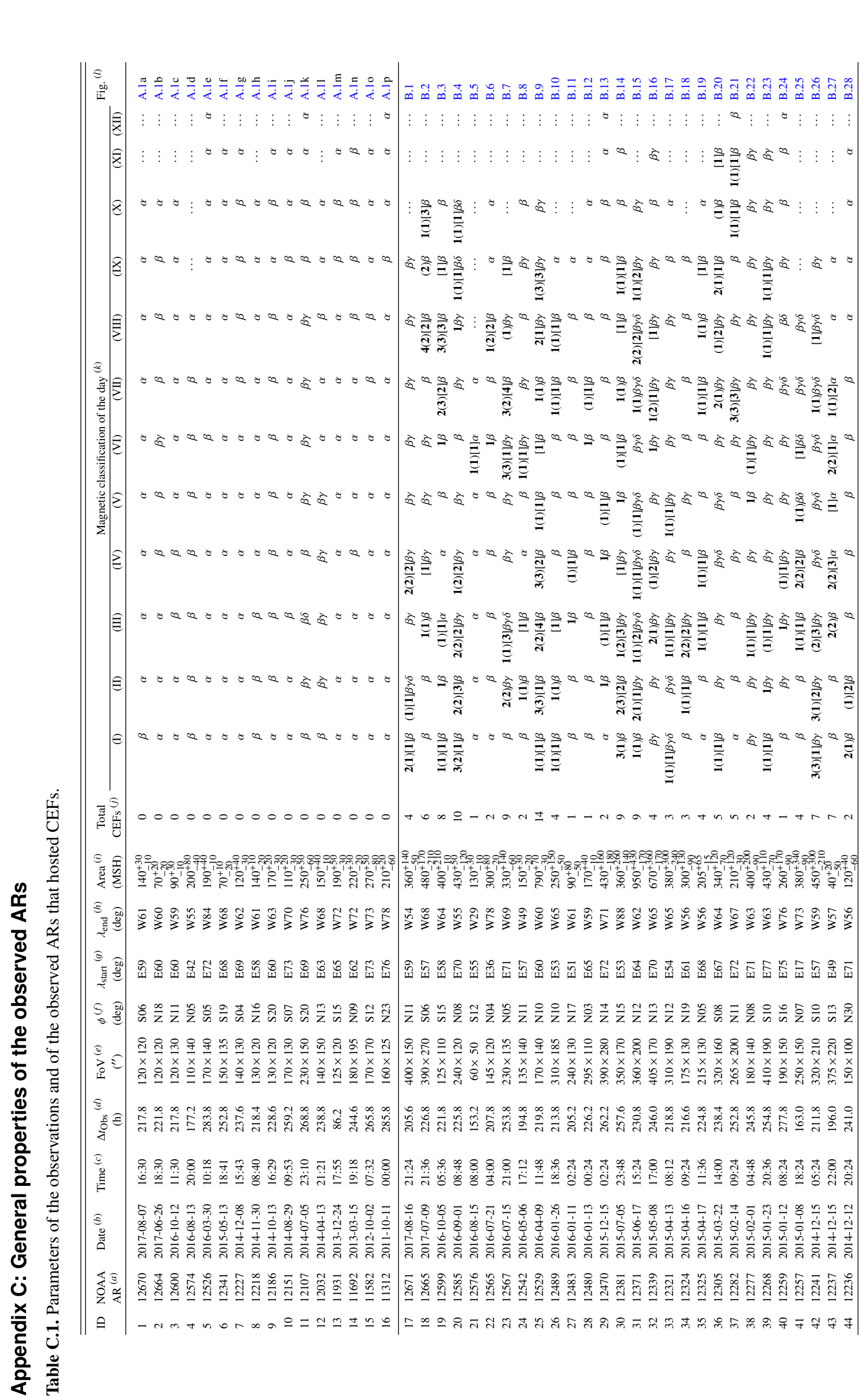

웡

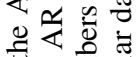

응 吾

\&

6.

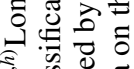

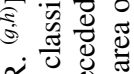

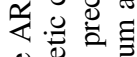

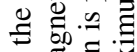

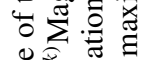

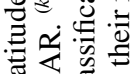

可

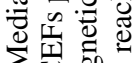

5 敋

过

훙

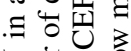

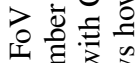

g艺品

녕

$\stackrel{0}{0}$

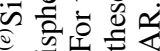

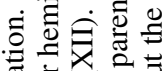

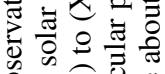

ô 0.

讨

to

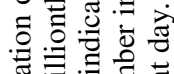

害家唐产

푱ㅎㅁ

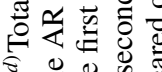

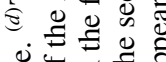

:

of

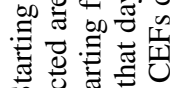

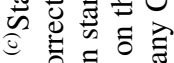

宅.

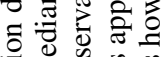

矛发占

㽞

웡 墕

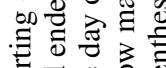

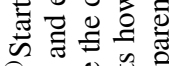

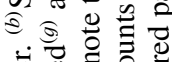

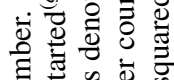

要

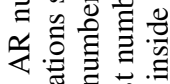

造词

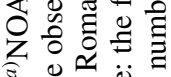

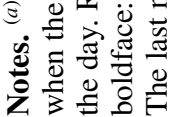

L1, page 37 of 38 


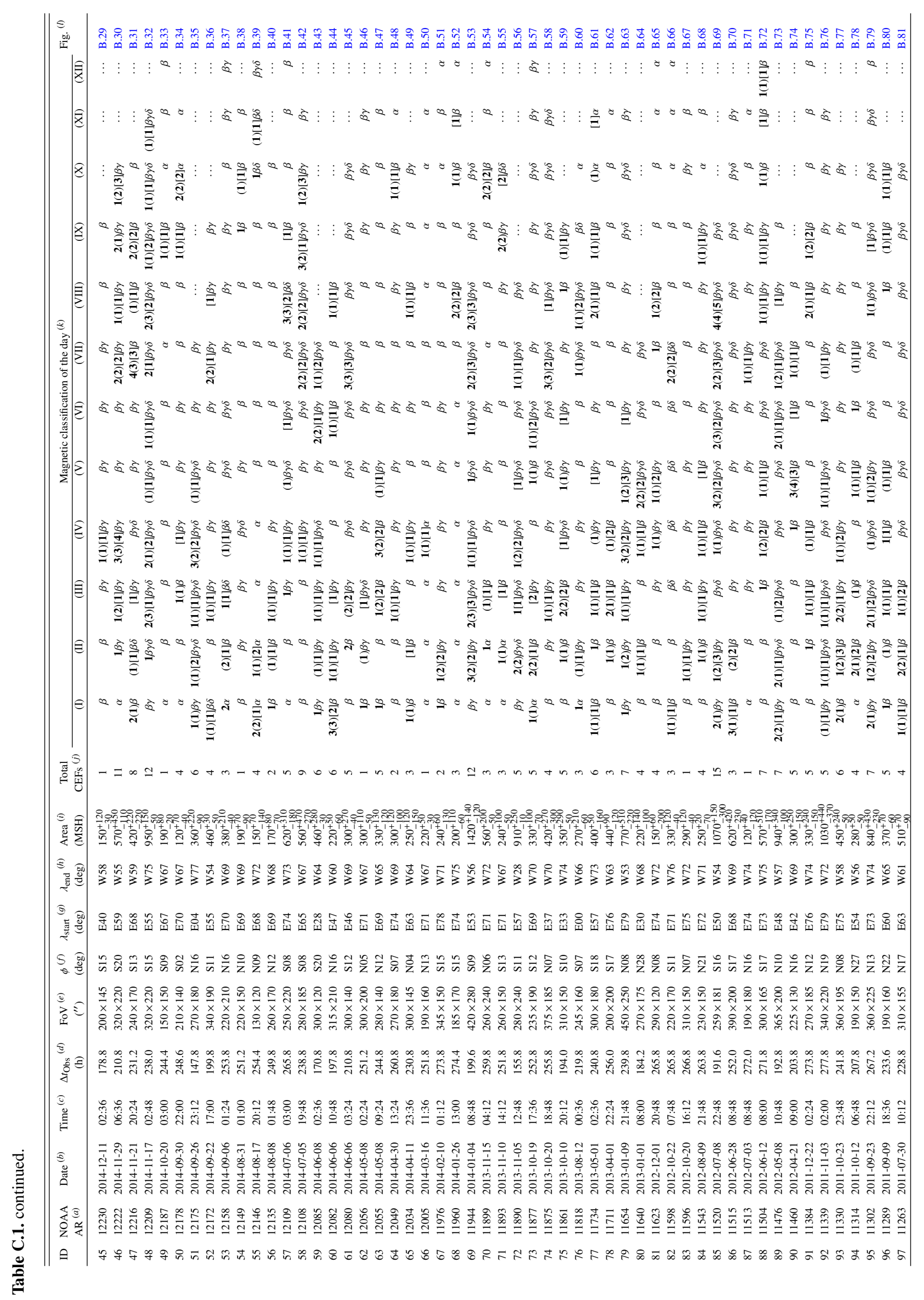

